\title{
Potential and sky coverage for off-axis fringe tracking in optical long baseline interferometry
}

\author{
Abdelkarim Boskri ${ }^{\circledR},{ }^{1,2 \star}$ Romain G. Petrov, ${ }^{2, \star}$ Thami El Halkouj, ${ }^{1}$ Massinissa Hadjara, ${ }^{3,4,5}$ \\ James Leftley, ${ }^{2}$ Zouhair Benkhaldoun ${ }^{\circledR},{ }^{1}$ Pierre Cruzalèbes, ${ }^{2}$ Aziz Ziad ${ }^{2}$ and Marcel Carbillet ${ }^{2}$ \\ ${ }^{1}$ LPHEA Laboratory, Oukaimeden Observatory, Cadi Ayyad University/FSSM, BP 2390, Marrakesh, Morocco \\ ${ }^{2}$ Observatoire de la Côte d'Azur, Université de la Côte d'Azur, CNRS, Laboratoire Lagrange, Parc Valrose, Bât. H. Fizeau, F-06108 Nice, France \\ ${ }^{3}$ Chinese Academy of Sciences South America Center for Astronomy, National Astronomical Observatories, CAS, Beijing 100101, China \\ ${ }^{4}$ Departamento de Astronomía, Universidad de Chile, Casilla 36-D, Santiago 7550000, Chile \\ ${ }^{5}$ Centre de Recherche en Astronomie, Astrophysique et Géophysique (CRAAG), Route de l'Observatoire, B.P. 63, Bouzareah, 16340, Alger, Algeria
}

Accepted 2021 May 20. Received 2021 May 18; in original form 2020 December 1

\begin{abstract}
A B S T RACT
The spectacular results provided by the second-generation VLTI instruments GRAVITY and MATISSE on active galactic nuclei (AGN) trigger and justify a strong increase in the sensitivity limit of optical interferometers. A key component of such an upgrade is off-axis fringe tracking. To evaluate its potential and limitations, we describe and analyse its error budget including fringe sensing precision and temporal, angular and chromatic perturbations of the piston. The global tracking error is computed using standard seeing parameters for different sites, seeing conditions and telescope sizes for the current GRAVITY Fringe Tracker (GFT) and a new concept of Hierarchical Fringe Tracker. Then, it is combined with a large catalogue of guide star candidates from Gaia to produce sky coverage maps that give the probability to find a usable off-axis guide star in any part of the observable sky. These maps can be used to set the specifications of the system, check its sensitivity to seeing conditions, and evaluate the feasibility of science programs. We check the availability of guide stars and the tracking accuracy for a large set of 15799 Quasars to confirm the feasibility of a large program on Broad Line Regions in the $K$ band with the GFT and show how it can be extended to the $L, M$, and $N$ bands. Another set of 331 well-characterized nearby AGNs shows the high potential of MATISSE for imaging and characterization of the dust torus in the $N$ band under off-axis tracking on both Unit Telescopes and Auxiliary Telescopes.
\end{abstract}

Key words:

atmospheric effects - instrumentation: high angular resolution - techniques: interferometric - galaxies: active - quasars: supermassive black holes.

\section{INTRODUCTION}

The second-generation VLTI general user focal instruments, GRAVITY (Gravity Collaboration 2017b) and MATISSE (Lopez et al. 2014), which are now in operation, represent a major turning point for Optical Long Baseline Interferometry. Both instruments combine either the four VLTI UTs (8 m 'Unit Telescopes') or the four VLTI ATs $(1.8 \mathrm{~m}$ 'Auxiliary Telescopes') and allow spectro-interferometric imaging with the resolution of a $130 \mathrm{~m}$ telescope in their respective spectral domains: the $K$ band (2-2.4 $\mu \mathrm{m}$ ) for GRAVITY and the $L, M$, and $N$ bands ( 3 $-13 \mu \mathrm{m})$ for MATISSE. GRAVITY has been operational since 2016 and has presented a set of breakthrough results on active galactic nuclei (AGN; Gravity Collaboration 2018, 2020b), extrasolar planets (Gravity Collaboration 2020a), Young Stellar Objects (YSOs), and our Galactic Centre. MATISSE saw its first light on sky in 2018 and is now publishing its first results on YSO (Matter, Pignatale \& Lopez 2020; Varga et al., 2021), exozodiacal light (Kirchschlager et al. 2020), AGNs, and Stellar Physics. Spectacular results are obtained on a small set of targets that

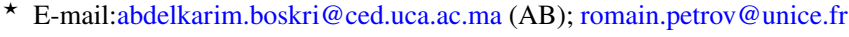
(RGP)
}

illustrate the potential of the VLTI for the study of protoplanetary discs and the co-evolution between supermassive black holes (SMBH) and their host galaxy. However, to be real game-changers such results have to be repeated on a large number of targets with a sufficient range of different physical conditions. Then the VLTI meets its sensitivity limit. In spite of breakthrough sensitivities with GRAVITY that allows routine high-quality fringe tracking up to $\mathrm{K}=10.5$ with $U T s$, as well as with MATISSE that can observe $20 \mathrm{mJy}$ sources in $L$, observations with the UTs are limited to a few dozen bright AGNs and even fewer for which we can obtain the size and kinematics of the Broad Line Regions (BLR). That strongly limits the number of objects in which we can measure the mass of the SMBH, the distance to the AGN (Wang et al. 2020a), or for which we can obtain detailed thermal images of the very complex dust distribution, which feeds the SMBH but also impacts star formation near the galactic centres. In the case of protoplanetary discs, we need detailed images of fine structures at high spectral resolution in quite resolved discs. This requires the use of the relocatable VLTI ATs. With the current sensitivity limits, this is again restricted to a small number of targets with a limited range of central star masses.

This situation has triggered the GRAVITY+ proposal (Eisenhauer 2019) and a new generation fringe tracker (FT) proposal (Petrov et al. 2019) intended to drastically improve the sensitivity limit of the VLTI. 
GRAVITY contains two instruments, a high-performance FT (the GRAVITY fringe tracker [GFT], the best FT in operation) that can work on an off-axis star and a science instrument that can reach a magnitude $\mathrm{K}=17$ (Gravity Collaboration 2017a) using long exposure integration when the GFT allows. The GFT can also be used to stabilize the fringes for MATISSE in the so-called GRA4MAT (GRAVITY for MATISSE) mode. The current main limitation both for GRAVITY and MATISSE is due to the aging adaptive optics (AO) that feed the VLTI beams on the UTs.

This sets a strong $R$ band limit to all VLTI programs and has a strong impact on the performances of the GFT on UTs, a point that will be briefly discussed in this paper in Section 2.5. Two other limitations to the off-axis fringe tracking are the very limited ( 8 arcsecond) separation currently possible between the off-axis tracking star and the science target and the relatively high level of vibrations in the VLTI, mainly with the UTs. The main goal of GRAVITY+ is to radically improve the UT AO used for the VLTI by replacing them with a new system with more actuators coupled to a Laser Guide Star. The second important goal is to improve the off-axis fringe tracking capability by increasing the possible separation between the off-axis guide star and the science target. GRAVITY+ will also work on the active damping of vibrations and on other features that can increase the GFT sensitivity.

Off-axis fringe tracking immediately raises the sky coverage issue. The sky coverage is the probability to find a usable off-axis guide star close enough to science target anywhere on the sky. It is limited by the sensitivity of the FT and by the angular variations of the atmospheric Optical Path Difference (OPD), which is called the atmospheric piston when it is averaged over each full individual aperture. The difference between the piston measured on the guide star and the piston that affects the science source is called the anisopistonic error and the acceptable angle between guide star and observed target is called the isopistonic angle, by analogy with the isoplanatic angle that affects higher-order AO or wide-field high angular resolution observations through the atmosphere. Estimates of the sky coverage are often based on a fringe tracking limiting magnitude combined with an isopistonic angle. The sky coverage is then the probability to find a guide star brighter than the limiting magnitude within the isopistonic radius. Here we use a more complete definition based on an analysis of the full-error budget for off-axis tracking that includes the fringe sensing precision, the servo loop delay and error, the anisopistonic error, and the chromatic piston error that appears when we are fringe tracking at a certain wavelength and observe in another. We consider the interaction between all these terms, although with some simplifications, to establish a more realistic model of the off-axis fringe tracking precision as a function of the angular separation. We develop analytical computations of the main error terms with parameters directly related to the standard seeing parameters that are measured at Paranal. Then we use a large catalogue of more than $8 \times 10^{8}$ guide star candidates, extracted from the $2^{\text {nd }}$ Gaia data release, to evaluate the sky coverage for different telescope sizes and seeing conditions for observations with GRAVITY ( $K$ band), $\operatorname{MATISSE}(L, M$, and $N$ bands) and with a potential new instrument in the $J$ band.

We consider two FTs. The GFT, as it is described in Lacour et al. (2019), is used to validate our fringe sensing estimates and to predict the performance of GRAVITY with the GRAVITY+ upgrade but with its unchanged internal FT. We consider also a new generation FT with a broader spectral domain, a slightly increased transmission and an innovative Hierarchical Fringe Tracking (HFT) concept (Petrov et al. $2014,2016,2019$ ) to evaluate the gain that could be expected from such an additional upgrade.

To be more specific than with general sky coverage maps, we consider with more detail two possible observing programs on AGNs, after summarizing their goals and methods. We define a large list of more than 15000 Quasars extracted from the Vista Hemisphere Survey and we use it to evaluate the number of targets accessible to a large BLR program on GRAVITY. A shorter list of 331 well-studied nearby AGNs, set up by Asmus et al. (2020), is used to investigate the potential of MATISSE in the GRAVITY+ context for detailed studies of AGN dust tori.

Our work on sky coverage and isopistonic errors started well before the GRAVITY+ project. We first investigated the issue in the context of optical interferometry in Antarctica (Elhalkouj et al. 2006), which triggered the parametric analysis of the anisopistonic error that we use here (Elhalkouj et al. 2008). Our isopistonic angle estimates are based on on-site testing campaigns with the Generalized Seeing Monitor (GSM) including two campaigns at Paranal (1998 and 2007) and one campaign at Dome $\mathrm{C}$. We have combined our analytic computation of the anisopistonic error with the statistics obtained on all these sites on the Fried parameter, the coherence time, the isoplanatic angle, and the outer scale, to produce the set of isopistonic angle statistics of Ziad et al. (2016) that is used in the present paper.

In the following, we first present the components of the error budget of an off-axis FT (Section 2.1), then we discuss the measurement errors on phase delay (Section 2.2) and group delay (Section 2.8), first for the GFT (Section 2.4). Then we briefly present the HFT (Section 2.7) and its estimated performances. We discuss the anisopistonic error (Section 2.9), the servo loop error (Section 2.10), and the optimum frame time set by its combination with the sensing error (Section 2.11). A note about the chromatic OPD error (Section 2.12) closes our estimation of the off-axis error analysis. Then we compute sky coverage maps for VLTI UTS (Section 3.3) and VLTI ATS (Section 3.4) after a general presentation of our sky coverage computation methodology (Section 3.2). As an example of the application of our methodology, we investigate the potential of off-axis tracking for interferometric observations of AGNs, briefly presented in Section 4 and we consider the potential of GRAVITY+ for a large program on BLR (Section 4.1) and the potential of MATISSE for several types of measures on dust torus, including imaging (Section 4.3). We finally briefly consider the impact of our analysis on the feasibility of the Planet Formation Imager (PFI; Monnier et al. 2018) that is the new generation interferometric project intended for high-resolution images of planetary formation down to the Hill sphere of giant planets. We consider the main goal of PFI that is YSOs observed in the $N$ band and its capacity to observe AGNs both in a Paranal site and at Dome C.

\section{OFF-AXIS FRINGE TRACKING IN OPTICAL IN TER F EROMETRY}

An optical interferometer produces a Young fringe pattern for each one of its baselines. These patterns, called interferograms, can be merged in 'global' beam combiners or analysed separately in 'pairwise' beam combiners. For each baseline $i j$ between the apertures $T_{i}$ and $T_{j}$, the Fourier transform of the interferograms yields the measured complex coherent flux

$$
\begin{aligned}
C_{i j}(\lambda) & =\sqrt{n_{* i}(\lambda) n_{* j}(\lambda)} V_{* i j}(\lambda) e^{i \varphi_{* i j}(\lambda)} V_{I i j}(\lambda) e^{i \varphi_{I i j}(\lambda)} \\
& =V_{C i j}(\lambda) e^{i \varphi_{C i j}}(\lambda)
\end{aligned}
$$

where $n_{* i}(\lambda)$ is flux received from $T_{i}$ in the interferogram, $V_{* i j}(\lambda) e^{i \varphi_{* i j}(\lambda)}$ is the complex visibility of the source and $V_{I i j}(\lambda) e^{i \varphi_{I i j}(\lambda)}$ is the interferometer complex visibility. The atmospheric turbulence and the interferometer vibrations introduce rapid variations of the OPD between the two beams, which affect the interfer- 
ometer phase

$\varphi_{I i j}(\lambda, t)=\varphi_{S i j}(\lambda)+\frac{2 \pi}{\lambda} p_{i j}(t)$,

where $\varphi_{I i j}(\lambda, t)$ is a fixed (or slowly varying) instrumental phase and $p_{i j}(t)$ is the instantaneous value of the OPD between $T_{i}$ and $T_{j}$, called the piston. The typical time needed to observe piston variations larger than $\frac{\lambda}{10}$ is called the piston coherence time $\mathcal{T}_{p}(\lambda)$. The atmospheric turbulence sets seeing dependent piston coherence time values ranging typically from $15 \mathrm{~ms}$ in the $J$ band at $1.25 \mu \mathrm{m}$ to $200 \mathrm{~ms}$ in the $N$ band at $11 \mu \mathrm{m}$. These typical values are from the AMBER (Petrov et al. 2007), MIDI (Leinert et al.2004), and MATISSE (Petrov et al. 2020) experience on the VLTI. The relevant values for the $K$ band used in this paper are discussed in Sections 2.10 and 2.11. If the piston is not corrected, the average $\left\langle e^{i \varphi_{* i j}}\right\rangle$ rapidly tends to zero for exposures longer than $\mathcal{T}_{p C}(\lambda)$. Then, it is only possible to record short exposures and to compute piston independent quantities such as the squared modulus of the coherent flux $\left|C_{i j}\right|^{2}$. This limits the sensitivity of interferometric instruments, for example to $\mathrm{K}<10$ and $\mathrm{N}<7$ on $U T$ s for $A M B E R$ and MIDI.

The device used to measure and correct in real-time the interferometric pistons $p_{i j}(t)$ is called a FT that is the AO system specific to a long-baseline interferometer. As there is no practical way to measure the contribution of the atmosphere to $p_{i j}(t)$ on artificial sources, the piston must be deduced from the coherent flux phase $\varphi_{I i j}(t)$ measured on the observed source itself (on-axis fringe tracking) or on a nearby guide star (off-axis fringe tracking).

In practice, we measure the variation of the coherent flux phase with time:

$$
\begin{aligned}
\varphi_{C i j}(\lambda, t+\mathrm{d} t)-\varphi_{C i j}(\lambda, t) & =\arg \left[C_{i j}(\lambda, t+\mathrm{d} t) \cdot C_{i j}^{*}(\lambda, t)\right] \\
& \simeq \frac{2 \pi}{\lambda}\left[p_{i j}(t+\mathrm{d} t)-p_{i j}(t)\right],
\end{aligned}
$$

if we assume that all other phase terms vary much slower than the piston. We actively correct this measured variation of the piston to maintain it constant. This variation of the phase with time is called phase delay. It is directly extracted from the phase of the measured coherent flux. The phase delay is defined modulo $2 \pi$ and measures the piston with a $k \lambda$ ambiguity. The absolute value of the piston can be deduced from the variation of the phase with wavelength $\lambda$ or wave number $\sigma=\frac{1}{\lambda}$,

$$
\begin{aligned}
d \varphi_{C i j}(\sigma, t) & =\arg \left[C_{i j}(\sigma+\mathrm{d} \sigma, t) \cdot C_{i j}^{*}(\sigma, t)\right] \\
& =\left[\mathrm{d} \varphi_{* i j}(\sigma)+\mathrm{d} \varphi_{S i j}(\sigma)-2 \pi p_{i j}(t)\right] \mathrm{d} \sigma .
\end{aligned}
$$

The slope of the coherent flux phase as a function of the wavenumber $\frac{\mathrm{d} \varphi_{C i j}(\sigma, t)}{\mathrm{d} \sigma}$ is called the group delay. It merges the absolute piston $p_{i j}(t)$ with instrumental and source terms. It could be used for fringe tracking, but it is usually not accurate enough to control the piston within a fraction of a wavelength at high frequency. However, it is a critical component of the robustness of a FT as it is used to acquire and reacquire the fringes when the tracking is lost and to maintain the fringes near the centre of the coherence length. To make an analogy with the optics of single large apertures, the group delay tracking is the active optics system that allows setting the working point of the interferometer, while the phase delay tracking is the AO system that allows correcting the effects of atmospheric turbulence and to make long exposures for high sensitivity observations.

The most efficient, state of the art, FT in operation is the GFT (Lacour et al. 2019). It can currently track up to magnitudes $K=9.5$ with the $1.8 \mathrm{~m}$ diameter $A T s$ and $\mathrm{K}=10.5$ with the $8 \mathrm{~m}$ diameter
UTs. It can be used off-axis with so far a small separation between the science source and the guide star. Then it allows making long exposure observations on science targets as faint as $\mathrm{K} \simeq 17$ (Gravity Collaboration 2017a). The GRAVITY+ proposal for 'sky faint sources VLTI observations'(Eisenhauer 2019) includes plans to increase the GFT sensitivity on UTS and allowing off-axis tracking with much larger separations is a part of it that already went through its first tests.

Several authors (Meisner, Jaffe \& Le Poole 2012; Ireland 2019) have proposed alternative FT designs. Below we will consider more in detail the Hierarchical Fringe Tracker (HFT; Petrov et al. 2016, 2019) that we have designed to be intrinsically more sensitive than the GFT. In the next subsection, we discuss first the parameters and the computation of the sensitivity of a FT.

\subsection{Error budget of off-axis fringe tracker and sky coverage}

The quality of fringe tracking will depend on :

(i) The precision of the phase delay measurement (variance $\sigma_{D}^{2}$ ) that, for a given design, is a function of the the number of detected photons collected from the guide source in each frame with Detector Integration Time $\mathcal{T}_{F}$.

(ii) The error introduced by the servo loop (variance $\sigma_{L}^{2}$ ) that applies an OPD correction sometime after its measurement. It is dominated by the seeing dependent temporal variation of the atmospheric piston over the FT period $\mathcal{T}_{L}$, if the telescope and interferometer mechanical vibrations are properly damped.

(iii) The anisopistonic error (variance $\sigma_{A}^{2}$ ) appears because the guide star and the observed target are not seen through the same atmospheric turbulence. For given seeing and site conditions, it depends on the angular separation between the two sources.

(iv) The chromatic phase delay difference (variance $\sigma_{H}^{2}$ ) between the wavelength used for guiding and for the observation that appears between near and mid-infrared (IR) mainly because of the variations of the differences in water vapor columns. This term is relevant only when FT and scientific observations are performed in different spectral bands.

If we have a specification $N_{\text {spec }}$ to reduce the fringe tracking residual (variance $\sigma_{T}^{2}$ ) below $\lambda_{o} / N_{\text {spec }}$ at the wavelength of observation $\lambda_{o}$, it writes:

$\sigma_{T}^{2}\left(\lambda_{o}\right)=\sigma_{D}^{2}\left(\lambda_{o}\right)+\sigma_{L}^{2}\left(\lambda_{o}\right)+\sigma_{A}^{2}\left(\lambda_{o}\right)<\left(\frac{\lambda_{o}}{N_{\text {spec }}}\right)^{2}$.

If we are tracking at a wavelength $\lambda_{t} \neq \lambda_{o}$ we have a specification on the fringe tracking quality and one of the final tracking residual,

$\sigma_{F}^{2}\left(\lambda_{t}\right)=\sigma_{D}^{2}\left(\lambda_{t}\right)+\sigma_{L}^{2}\left(\lambda_{t}\right)<\left(\frac{\lambda_{t}}{N_{\text {tra }}}\right)^{2}$,

to ensure that the loop remains closed and that we do not have fringe jumps that would destroy the information at $\lambda_{o}$,

$\sigma_{T}^{2}\left(\lambda_{o}\right)=\sigma_{F}^{2}\left(\lambda_{t}\right)+\sigma_{H}^{2}\left(\lambda_{t}, \lambda_{o}\right)+\sigma_{A}^{2}\left(\lambda_{o}\right)<\left(\frac{\lambda_{o}}{N_{\text {spec }}}\right)^{2}$,

where $\sigma_{H}^{2}\left(\lambda_{t}, \lambda_{o}\right)$ is the variance of the phase delay difference between $\lambda_{t}$ and $\lambda_{o}$ on the guide star and $\sigma_{A}^{2}\left(\lambda_{o}\right)$ is the variance of the anisopistonic error at the wavelength $\lambda_{o}$.

The sky coverage near coordinates $(\alpha, \delta)$ is the probability to find at least one usable guide star that complies with the conditions set by equations ( 5 or 7 ) for any science target in that region of the sky. This definition is more restrictive and more accurate than the often used 'the probability to find a guide star closer than the Isopistonic angle $\theta_{p}$ and brighter than the fringe tracking magnitude limit $M_{\text {lim }}$ '. Indeed, if 
the definition of the Isopistonic angle is $\sigma_{A}\left(\theta_{p}\right)=\left(\frac{\lambda_{o}}{N_{\text {spec }}}\right)^{2}$, a guide star at the distance $\theta_{p}$ is usable only if $\sigma_{D}^{2}+\sigma_{L}^{2}=0$ and a guide star of magnitude $M_{\text {lim }}$ such as $\sigma_{D}^{2}\left(M_{l}\right)+\sigma_{L}^{2}=\left(\frac{\lambda_{o}}{N_{\text {spec }}}\right)^{2}$ is usable only if the distance to the target is zero. Furthermore, we have to consider the variations of seeing and hence $\sigma_{D}^{2}, \sigma_{L}^{2}$, and $\sigma_{A}^{2}$ with the minimum zenith distance of the target.

\subsection{Phase delay measurement}

The phase delay in each frame is the phase $\varphi_{C i j}(\lambda)$ of the complex coherent flux $C_{i j}(\lambda)$ :

$\varphi_{C i j}(\lambda)=\arg \left\{\frac{\mathfrak{I}\left[C_{i j}(\lambda)\right]}{\mathfrak{R}\left[C_{i j}(\lambda)\right]}\right\}$,

where $\Re$ and $\Im$ note the real and imaginary parts of the complex coherent flux.

The variance of the coherent flux is

$\operatorname{Var}\left[C_{i j}(\lambda)\right]=\operatorname{Var}\left(\mathfrak{I}\left[C_{i j}(\lambda)\right]\right)+\operatorname{Var}\left(\Re\left[C_{i j}(\lambda)\right]\right)$.

Assuming that the real and imaginary parts of the coherent flux are independent variables with the same variance, which would be the case for an ideal beam combiner and has been found to be a good approximation in real instruments, yields:

$\operatorname{Var}\left(\mathfrak{R}\left[C_{i j}(\lambda)\right]\right)=\operatorname{Var}\left(\mathfrak{I}\left[C_{i j}(\lambda)\right]\right)=\operatorname{Var}\left[C_{i j}(\lambda)\right] / 2$

Many authors (Petrov 1989; Vannier et al. 2006; Houairi et al. 2008) have computed the variance of the coherent flux in the presence of detector noise and source plus background photon noise. For a pairwise beam combiner using single mode spatial filters, this variance is given by:

$\operatorname{Var}\left[C_{i j}(\lambda)\right]=\frac{n_{* i}+n_{* j}+n_{B i}+n_{B j}}{N_{P} N_{\lambda}} \mathcal{T}_{F} \alpha^{2}+N_{X} \sigma_{R}^{2}$,

where $n_{* i}$ is the number of detected photons/s received from the telescope $T_{i}$ in the FT spectral band, $n_{B i}$ is the number of detected background photons/s in the beam $i$, in the same spectral band, at the entrance of the cold optics, $\mathcal{T}_{F}$ is the frame time, $N_{P}$ is the number of fringe tracking baselines involving each telescope, $N_{\lambda}$ is the number of spectral channels, $N_{X}$ is the number of pixels per spectral channel, $\sigma_{R}^{2}$ is the variance of the detector read-out noise, and $\alpha$ is the photon noise amplification factor observed in intensified detectors. Equation (9) simply expresses the fact that the coherent flux variance is the sum of the variance of the source and background photon noise and the detector read-out noise on all used pixels.

When we are close to the centre of the coherence length (i.e. the fringes have been acquired and centred by the group delay tracker), we can coherently add the different spectral channels (i.e. use an average of the interferograms over the available bandpass) and the variance of the broadband coherent flux becomes:

$\operatorname{Var}\left(C_{i j L}\right)=\frac{n_{* i}+n_{* j}+n_{B i}+n_{B j}}{N_{P}} \mathcal{T}_{F} \alpha^{2}+N_{\lambda} N_{X} \sigma_{R}^{2}$.

The average coherent flux is given by

$\left\langle C_{i j L}\right\rangle=\frac{\mathcal{T}_{F} \sqrt{n_{* i} n_{* j}}}{N_{P}} V_{i j}$

where $V_{i j}$ is the visibility measured on the fringe tracking target. In single-mode instruments, the variations of $V_{i j}$ are strongly dominated by changes in the residual piston jitter:

$V_{I i j}=\exp \left[-2\left(\frac{\pi}{\lambda}\right)^{2}\left(\sigma_{V i j}^{2}+\sigma_{T i j}^{2}\right)\right]$, with $\sigma_{V}^{2}$ and $\sigma_{T}^{2}$ are the residual variances of the vibrational and atmospheric piston jitters.

When the coherent flux signal-to-noise ratio (SNR) is high, and the phase delay is small, equations ( 8 and 9 ) yield Petrov, Roddier \& Aime (1986),

$$
\begin{aligned}
\varphi_{C i j L} & =\frac{\mathfrak{J} C_{i j L}}{\mathfrak{R} C_{i j L}} \text {, and } \\
\sigma_{\varphi_{C i j L}} & =\frac{\sqrt{\operatorname{Var}\left(\mathfrak{J} C_{i j L}\right)}}{\left\langle\mathfrak{R} C_{i j L}\right\rangle}=\frac{1}{\sqrt{2} S N R_{C_{i j L}}} .
\end{aligned}
$$

We define the ratio:

$$
\begin{aligned}
Z_{i j L} & =\frac{\sqrt{\operatorname{Var}\left(\mathfrak{J} C_{i j L}\right)}}{\left\langle\mathfrak{R} C_{i j L}\right\rangle} \\
& =\frac{\sqrt{\left(n_{* i}+n_{* j}+n_{B i}+n_{B j}\right) \mathcal{T}_{F} \alpha^{2}+N_{X} N_{P} N_{\lambda} \sigma_{R}^{2}}}{\mathcal{T}_{F} \sqrt{2 n_{* i} n_{* j} V_{i j}}} \sqrt{N_{P}} .
\end{aligned}
$$

We assume that the two beams see comparable backgrounds $n_{B i} \simeq$ $n_{B j} \simeq n_{B}$. To express the unbalance between beams, we write $n_{* i}=$ $n_{*}(1-\gamma)$ and $n_{* j}=n_{*}(1+\gamma)$ where $n_{*}$ is the average source flux and $\gamma=\frac{n_{* j}-n_{*} i}{n_{* j}+n_{* i}}$ describes the source flux ratio between the two beams.

Then

$$
\begin{aligned}
Z_{i j L} & =\frac{\sqrt{\left(n_{*}+n_{B}\right) \mathcal{T}_{F} \alpha^{2}+\frac{N_{X} N_{P} N_{\lambda}}{2} \sigma_{R}^{2}}}{\mathcal{T}_{F} n_{*} V_{i j} \sqrt{1-\gamma^{2}}} \sqrt{N_{P}} \\
& =\frac{1}{\sqrt{2} S N R_{C_{i j L}}} .
\end{aligned}
$$

The flux unbalance is equivalent to a change in the instrument visibility, negligible for small flux differences $(\gamma \sim 0)$, and dramatic when one beam is lost $(\gamma=1)$. Note that if the collected flux varies rapidly within the frame time, the correct expression for $\gamma$ is given by an average of its instantaneous values:

$$
\gamma(t)=\frac{1}{\mathcal{T}_{F}} \int_{t}^{t+\mathcal{T}_{F}} \frac{n_{* j}\left(t^{\prime}\right)-n_{* i}\left(t^{\prime}\right)}{n_{* j}\left(t^{\prime}\right)+n_{* i}\left(t^{\prime}\right)} \mathrm{d} t^{\prime} .
$$

Micro cuts in the injection efficiency at different instants in each beam can result in strong SNR drops even if the average fluxes $\left\langle n_{* i}(t)\right\rangle$ and $\left\langle n_{* j}(t)\right\rangle$ are comparable.

The approximation $\sigma_{\varphi_{C i j L}}=\frac{\sqrt{\operatorname{Var}\left(\mathfrak{I} C_{i j L}\right)}}{\left\langle\mathfrak{R} C_{i j L}\right\rangle}=Z_{i j L}$ is considered in the literature as acceptable up to $Z_{i j L}=1 \mathrm{rad}$ for phase precision estimates. We found that this underestimates the phase delay error at low SNR with possibly significant impact on the actual fringe tracking limiting magnitude. Papoulis (1965) gives an expression of the probability density of $\phi=\arg (\mathfrak{I} / \mathfrak{R})$ as a function of the ratio $Z=\sqrt{\operatorname{Var}(\mathfrak{I})} /\langle\mathfrak{R}\rangle$, when $\mathfrak{I}$ and $\mathfrak{R}$ are two independent normal variables with the same variance $\operatorname{Var}(\mathfrak{I})=\operatorname{Var}(\mathfrak{R})$ and $\langle\mathfrak{I}\rangle=0$,

$$
f_{\phi}(\theta)=\left\{\begin{array}{ll}
\frac{e^{\frac{-1}{2 Z^{2}}}}{2 \pi}+\frac{\cos (\theta) e^{\frac{-\sin ^{2}(\theta)}{2 Z^{2}}}}{2 Z \sqrt{2 \pi}} & \\
\times\left[1+2 \operatorname{erf}\left(\frac{\cos (\theta)}{Z}\right)\right] ; & \text { if }|\theta| \leq \min (\kappa Z, \pi), \\
0 ; & \text { else }
\end{array},\right.
$$

where we have introduced the $\min (\kappa Z, \pi)$ limit to eliminate the incorrectbehaviour of thatPapoulisfunction near $\pm \pi$. The parameter $\kappa=$ 5 has been selected from numerical tests to allow a good representation of the actual probability density. 


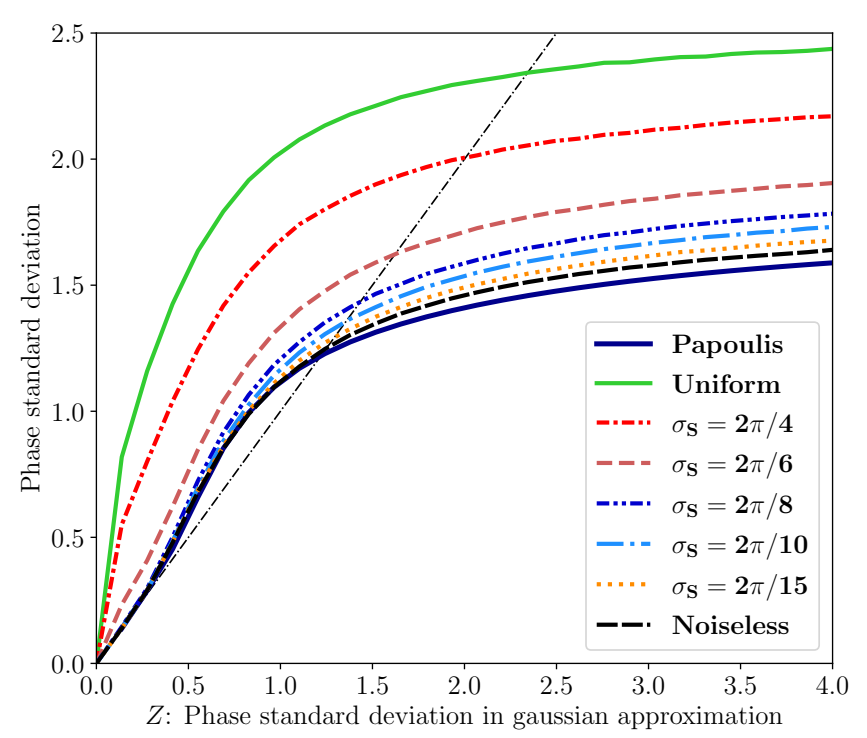

Figure 1. Standard deviation of the phase estimation error in one frame $\sigma_{\phi}$, as a function of $Z$ that is its value in the Gaussian approximation $\left(Z=1 / \sqrt{2 S N R_{C}}\right)$, for different statistics of the 'input' phase that has to be estimated. From top to bottom: input phases uniformly distributed between $\pm \pi$ (top, full green line), input phases with a normal distribution with standard deviations $\sigma_{S}=2 \pi / 4, \sigma_{S}=2 \pi / 6, \sigma_{S}=2 \pi / 8 ; \sigma_{S}=2 \pi / 10$; $\sigma_{S}=2 \pi / 15$, and finally $\sigma_{S}=0$ for the noiseless case (bottom, black dashed line). The thin straight line represents the Gaussian approximation.

When $Z \ll 1, f_{\phi}(\theta)$ is very close to a normal distribution with standard deviation $Z$, and when $Z>1, f_{\phi}(\theta)$ rapidly tends towards an uniform distribution between $-\pi$ and $+\pi$ with a standard deviation $\pi / \sqrt{3}$. The variance of $\arg (\mathfrak{I} / \mathfrak{R})$ is given by:

$\sigma_{\phi, Z}^{2}=\int_{-\pi}^{\pi} \theta^{2} f_{\phi, Z}(\theta) \mathrm{d} \theta$

The standard deviation $\sigma_{\phi}$ is a correct estimation of the error on a phase very close to zero. The function $\sigma_{\phi}(Z)$ is plotted in Fig. 1 that shows that $\sigma_{\phi}(Z) \simeq Z$ looks like an acceptable approximation up to $Z \sim 1.2$.

However, when it is applied to the non-zero phase $\theta$ of a noisy coherent flux, the estimator

$\theta^{\prime}=\arg \left[M \sin (\theta)+I_{N}, M \sin (\theta)+R_{N}\right]$,

is biased with a bias depending on $\theta$ and the coherent flux SNR. This is a mathematical bias entirely due to the non-linearity of the argument function for values that are far from zero. The probability density of the phase can be roughly divided in a narrow part centred around $\theta$ on top of broad part close to a uniform distribution between $\pm \pi$, as illustrated by Fig. 2, with an average shifted towards zero in proportion to the relative importance of the broad part, i.e. of the coherent flux SNR. Therefore, the phase is systematically underestimated. This bias could be evaluated and explicitly corrected with a certain cost in phase delay precision. It can also be implicitly corrected by the optimization of the gain of the fringe tracking loop as a function of coherent flux SNR. A full discussion of this mathematical bias is beyond the scope of this paper and here we only evaluate its global effect on the fringe detection SNR.

We compute the variance of the estimator $\theta_{Z}^{\prime}$ in equation (21) from a large number of values of $\theta^{\prime}$ for random values of $\theta, I_{N}$ and $R_{N}$. The phase $\theta$ to be estimated is a centred Gaussian random variable with variance $\sigma_{S}^{2} . I_{N}$ and $R_{N}$ are centred Gaussian random variables with variance 1 that represent the noise on the imaginary and real parts of the

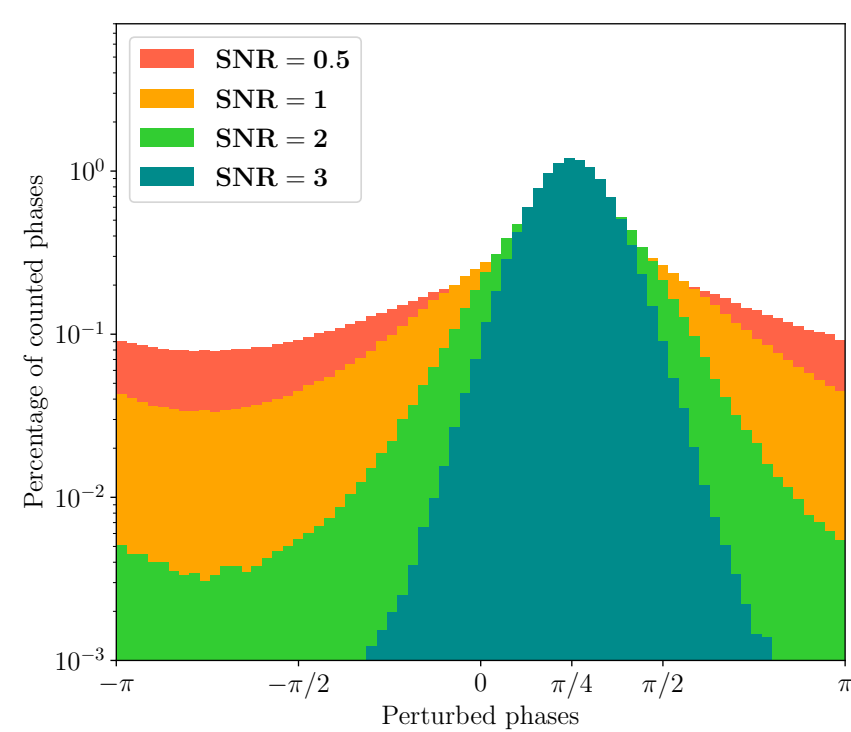

Figure 2. Histogram of the phase estimator of a coherent flux phase of $45^{\circ}$, for different values of the coherent flux SNR (from top to bottom): $0.5 ; 1 ; 2$; and 3 .

coherent flux. The parameter $M$ sets the value $Z=\frac{\sqrt{\operatorname{Var}(\mathfrak{J})}}{\langle\mathfrak{R}\rangle}=1 / M$. We compute the standard deviation of the estimation error

$\sigma_{\varphi, \sigma_{S}}^{2}(Z)=\left\langle\left(\theta_{Z}^{\prime}-\theta\right)^{2}\right\rangle$,

Fig. 1 shows $\sigma_{\varphi, \sigma_{S}}=f(Z)$ for various values of $\sigma_{S}$. The line $\sigma_{\varphi}(Z)=Z$ represents the Gaussian approximation of the phase delay error. For $\sigma_{S}=0$, we obtain the standard deviation of $f_{\phi}(\theta)$ : $\sigma_{\varphi, 0}(Z)=\sigma_{\phi}$ discussed above. For $\sigma_{S} \lesssim 8.5$, we have $\sigma_{\varphi, \sigma_{S}}(Z)=Z$ for $Z \leqslant 0.25$ and then $\sigma_{\varphi, \sigma_{S}}(Z)-Z$ grows with $\sigma_{S}$. For $\sigma_{S} \geq 8.5$, we have $\sigma_{\varphi, \sigma_{S}}(Z) \neq Z$ for all values of $Z$ which means that $\sigma_{\varphi}$ is not proportional to $1 / \sqrt{n_{*}}$ even at very high flux.

In a given frame, the fringe sensor has to estimate a phase delay $\theta \neq 0$ because the phase delay in the previous frame has been estimated with an error and corrected with an even larger error due to the variation of the atmospheric phase delay during the frame duration. The change in phase delay between measure and correction is what we have called the 'servo loop' error. Its value is discussed in Section 2.10. Here, we evaluate only its influence on the phase delay estimation error.

For given observing conditions and a given frame time $\mathcal{T}_{F}$, the servo loop error has a constant standard deviation $\sigma_{L}$. It will trigger an estimation error $\sigma_{\varphi, \sigma_{L}}(Z)$ that will add quadratically to the servo loop error in the next frame. To estimate this effect, we have computed numerically the series:

$\sigma_{\varphi, \sigma_{L}}(Z, N)=\sigma_{\varphi, \sigma_{N}}$ with $\sigma_{N}^{2}=\sigma_{\varphi, \sigma_{L}}^{2}(Z, N-1)+\sigma_{L}^{2}$

This series converges very rapidly, in less than 10 frames, towards an asymptotic value $\sigma_{\text {final }}\left(Z, \sigma_{L}\right)$ that is independent of the starting point of the series. This final phase delay estimation error is plotted in Fig. 3. We seethat for servolooperrorslargerthan $\sim 2 \pi / 8, \sigma_{\text {f inal }}\left(Z, \sigma_{L}\right)>$ $Z$ for any value of $Z \lesssim 2.5$. For smaller servo loop errors, the Gaussian approximation $\sigma_{\text {final }}\left(Z, \sigma_{L}\right)=Z$ is valid up to $Z \sim 0.25$ (phase delay $\mathrm{SNR}>4$ ) and increase much more rapidly with $Z$ after that value.

A phase delay $\mathrm{SNR}=1 \mathrm{rad}$ can be achieved for a coherent flux SNR 1 $(Z \sim 0.7)$ on a single-phase estimator. 


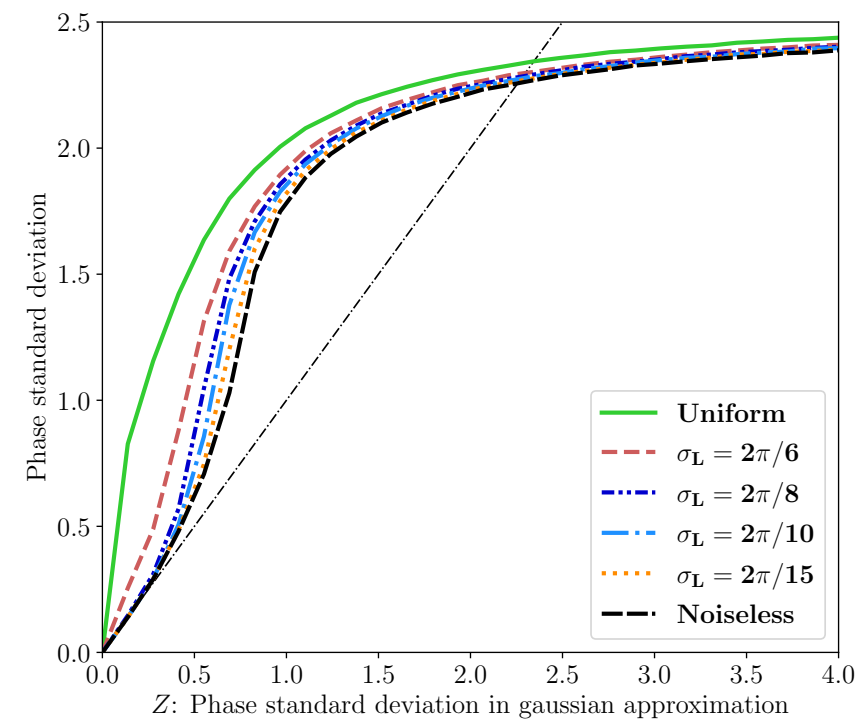

Figure 3. Asymptotic standard deviation of the phase estimation error after a few frames, for different values of the servo loop standard deviation $\sigma_{L}$. From bottom to top: $\sigma_{L}=0, \frac{2 \pi}{15} ; \frac{2 \pi}{10} ; \frac{2 \pi}{8} ; \frac{2 \pi}{6}$. The full green line represents the estimation error on a phase randomly distributed between $\pm \pi$.

\subsection{Phase delay estimators combining all baselines}

For a four telescope interferometer, we can retrieve only three combined phase delays from the three piston differences:

$\theta_{i j}=\frac{2 \pi}{\lambda}\left(P_{j}-P_{i}\right)$ with $i \in\{1,2,3\}$ and $j=i+1$.

We have a phase delay $b_{i j}$ and a phase delay measurement $m_{i j}$ for each baseline with:

$$
\left\{\begin{array}{l}
b_{12}=\theta_{12} \\
b_{13}=\theta_{12}+\theta_{23} \\
b_{14}=\theta_{12}+\theta_{23}+\theta_{34} \\
b_{23}=\theta_{23} \\
b_{24}=\theta_{23}+\theta_{34} \\
b_{34}=\theta_{34}
\end{array},\right.
$$

and, as in equation (21)

$m_{i j}=\arg \left[M_{i j} \sin \left(b_{i j}+\varphi_{n i j}\right)+I_{i j}, M_{i j} \cos \left(b_{i j}+\varphi_{n i j}\right)+R_{i j}\right]$,

$\varphi_{n i j}$ is the noise on the phase resulting from the error on the correction of the previous frame, $R_{i j}$ and $I_{i j}$ are the noise on the real and imaginary parts of the coherent flux of mean modulus $M_{i j}$.

The estimators $\theta_{i j}^{\prime}$ of the phase delay are the parameters that minimize the sum of square differences:

$\chi^{2}=\sum_{i=1}^{N_{T}-1} \sum_{j=i+1}^{N_{T}}\left(m_{i j}-b_{i j}^{\prime}\right)^{2}$,

with $b_{i j}^{\prime}$ deduced from the $\theta_{l k}^{\prime}$ using equations (25).

$$
\begin{aligned}
\chi^{2} & =\left(m_{12}-\theta_{12}^{\prime}\right)^{2}+\left(m_{13}-\theta_{12}^{\prime}-\theta_{23}^{\prime}\right)^{2} \\
& +\left(m_{14}-\theta_{12}^{\prime}-\theta_{23}^{\prime}-\theta_{34}^{\prime}\right)^{2}+\left(m_{23}-\theta_{23}^{\prime}\right)^{2} \\
& +\left(m_{24}-\theta_{23}^{\prime}-\theta_{34}^{\prime}\right)^{2}+\left(m_{34}-\theta_{34}^{\prime}\right)^{2} .
\end{aligned}
$$

The three equations setting the minimum value of $\chi^{2}$ for each of the parameters

$$
\frac{\mathrm{d} \chi^{2}}{\mathrm{~d} \theta_{l k}^{\prime}}=0
$$

write

$\left[\begin{array}{l}\theta_{12}^{\prime} \\ \theta_{23}^{\prime} \\ \theta_{34}^{\prime}\end{array}\right]\left[\begin{array}{lll}3 & 2 & 1 \\ 2 & 4 & 2 \\ 1 & 2 & 3\end{array}\right]=\left[\begin{array}{c}m_{12}+m_{13}+m_{14} \\ m_{13}+m_{14}+m_{23}+m_{24} \\ m_{14}+m_{24}+m_{34}\end{array}\right]$,

hence, from the inversion of that system matrix

$\left[\begin{array}{c}\theta_{12}^{\prime} \\ \theta_{23}^{\prime} \\ \theta_{34}^{\prime}\end{array}\right]=\frac{1}{4}\left[\begin{array}{ccc}2 & -1 & 0 \\ -1 & 2 & -1 \\ 0 & -1 & 2\end{array}\right]\left[\begin{array}{c}m_{12}+m_{13}+m_{14} \\ m_{13}+m_{14}+m_{23}+m_{24} \\ m_{14}+m_{24}+m_{34}\end{array}\right]$,

or

$\theta_{12}^{\prime}=\frac{2 m_{12}+\left(m_{13}-m_{23}\right)+\left(m_{14}-m_{24}\right)}{4}$

$\theta_{23}^{\prime}=\frac{2 m_{23}+\left(m_{13}-m_{12}\right)+\left(m_{24}-m_{34}\right)}{4}$,

$\theta_{34}^{\prime}=\frac{2 m_{34}+\left(m_{14}-m_{13}\right)+\left(m_{24}-m_{23}\right)}{4}$

In each one of these equations, we combine three estimators of the phase delay. For example $m_{12},\left(m_{13}-m_{23}\right)$, and $\left(m_{14}-m_{24}\right)$ are all estimators of $\theta_{12}$. When one of these estimators jumps by $2 \pi$, the combined estimator $\theta_{34}^{\prime}$ will jump by $\pi / 2$ or by $\pi$. To avoid this partial wrapping of the phase we write:

$$
\begin{aligned}
& \theta_{12}^{\prime}=\left(\frac{2 m_{12}+\left(m_{13}-m_{23}+\pi\right)_{[2 \pi]}+\left(m_{14}-m_{24}+\pi\right)_{[2 \pi]}-2 \pi}{4}+\pi\right)_{[2 \pi]}-\pi \\
& \theta_{23}^{\prime}=\left(\frac{2 m_{23}+\left(m_{13}-m_{12}+\pi\right)_{[2 \pi]}+\left(m_{24}-m_{34}+\pi\right)_{[2 \pi]}-2 \pi}{4}+\pi\right)_{[2 \pi]}-\pi, \\
& \theta_{12}^{\prime}=\left(\frac{2 m_{34}+\left(m_{14}-m_{13}+\pi\right)_{[2 \pi]}+\left(m_{24}-m_{23}+\pi\right)_{[2 \pi]}-2 \pi}{4}+\pi\right)_{[2 \pi]}-\pi
\end{aligned}
$$

The standard error on the phase delay estimation will then be

$\sigma_{\theta_{i j}}^{2}=\operatorname{Var}\left(\theta_{12}^{\prime}-\theta_{i j}\right)$.

As we have seen above for the phase delay estimator on a single baseline, $\sigma_{\theta_{i j}}^{2}$ depends on the coherent flux $S N R_{C}=\frac{M_{i j}}{\sqrt{\operatorname{Var}\left(I_{i j}\right)}}=\frac{1}{Z}$ and from the variance of $\theta_{i j}$. We apply the same iterative simulation as for a single-phase delay and the result is given in Fig. 4.

The final result is that the Gaussian approximation is not valid below $S N R_{C}<3(Z=0.5)$. A phase delay estimation error of $0.4 \mathrm{rad}$, corresponding to $\frac{\lambda}{15}$, can be achieved for $S N R_{C} \sim 2$ for $\sigma_{L}=\frac{2 \pi}{15}$.

Equation (33) and the numerical computation described above give the standard deviation of the phase delay estimator as a function of $\mathrm{Z}=$ $1 /\left(\sqrt{2} S N T R_{C}\right)$ described in equation (17). We perform a polynomial fit of the result given by equation (33) up to $\sigma_{\theta_{i j}}=1$ :

$\sigma_{\theta_{i j}}\left(Z_{i j L}\right)=Z_{i j L}+a_{1} Z_{i j L}^{3}+a_{5} Z_{i j L}^{5}+a_{7} Z_{i j L}^{7}+a_{9} Z_{i j L}^{9}$,

with $a_{1}, a_{3}, a_{5}, a_{7}$, and $a_{9}$ are fixed coefficients (for a given FT) that describe the bias introduced by the estimator argument applied to a noisy complex coherent flux. Combining equations (17 and 34) yields the phase delay measurement error $\sigma_{t}$.

\subsection{Phase delay measurement in the GFT}

The GFT (Lacour et al. 2019) is a pairwise FT using an integrated optics beam combiner that splits the signal for each of the six baselines into four pixels that sample the phases $0, \pi / 2, \pi$, and $3 \pi / 2$ in the so-called 'ABCD' scheme. It is important to remember that integrated optics and single-mode fibers yield a spatial filtering of each telescope beam 


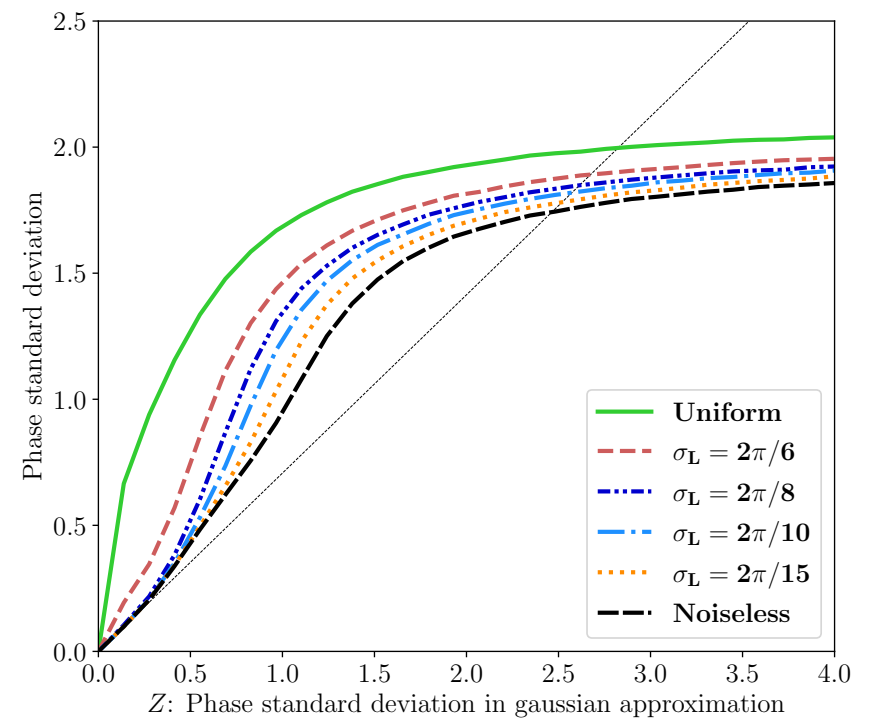

Figure 4. Standard deviation of the three independent phase delays computed from the six baselines as a function of $Z$ that is the Gaussian approximation for the phase delay error per baselines. The parameters are the same as in Fig. 3.

that reduces the dependence of the measured instrumental contrast and SNR to only two factors: piston jitters between beams, which affect the term $V_{I i j}$ of equation (17), and fluctuations of the ratios of flux injected in each beam that set the term $\gamma$ in the same equation. Each output is dispersed over six spectral channels covering the $K$ band. It uses a SAPHIRA detector (Finger et al. 2016) with a very low read-out noise $\left(\sigma_{R} \sim 0.7 e^{-}\right)$and a noise amplification factor $\alpha \sim 1.5$. The measured overall transmission of the VLTI and the GFT, including the detector efficiency, is $\sim 1$ per cent. The six phase delay measurements on each baseline are combined to obtain the three phase delays corresponding to the OPD difference between the four telescopes. For phase delay measurements, the interferograms in the six spectral channels are added after the correction of the known chromatic OPD. For group delay measurements, the spectral channels are processed separately and combined in a group delay estimator that uses 40 individual frames.

Equation (17) allows us to express $Z_{i j L}$ as a function of the number $n_{*}$ per detected photons per telescope per second. Here, we assume that $Z_{i j L}-Z$ is the same for all baselines. Then we use equation (33) and the numerical computation described above to obtain the variance $\sigma_{\varphi_{\theta i j}}^{2}\left[Z\left(n_{*}\right)\right]$. With the parameters given in Table 1 and for $V_{I} \sqrt{1-\gamma^{2}} \sim 0.8$, which corresponds to the VLTI with ATs that has moderate vibrations and flux imbalances, we obtain the phase delay $S N R=1 / \sigma_{\theta}$ as a function of $n_{*}$ shown in Fig. 5, where it has been plotted over the experimental data points copied from Lacour et al. (2019).

When $n_{*}$ is converted in $K$ band magnitude with $A T s$ using again the parameters in Table 1, the limiting magnitude that corresponds to a limit $S N R \sim 3$, i.e. $5 \cdot 10^{3}$ detected photons $/ \mathrm{s}$, is $\mathrm{K}=9.45$, that is indeed the GFT magnitude offered to users. This shows that our analytical SNR is in good agreement with the experimental results of the GFT with ATs.

\subsection{Maximum sensitivity of phase delay sensing}

Equation (17) and Table 1 set the conditions to optimize the limiting sensitivity of a phase delay tracker. For a given detector technology, i.e. fixed readout noise $\sigma_{R}$ and noise amplification factor $\alpha$, the first step is to
Table 1. Parameters for SNR and sky coverage estimate at the VLTI and for the various FTs.

\begin{tabular}{|c|c|c|c|c|}
\hline & \multicolumn{2}{|c|}{ GFT } & \multirow{2}{*}{$\begin{array}{c}\text { GFT+ } \\
\text { UTs }\end{array}$} & \multirow{2}{*}{$\begin{array}{l}\text { HFT } \\
\text { UTs }\end{array}$} \\
\hline & $A T s$ & $U T s$ & & \\
\hline Nbr. telescopes $N_{T}$ & \multicolumn{4}{|c|}{4} \\
\hline Detector & \multicolumn{4}{|c|}{ SAPHIRA } \\
\hline Read out noise $\sigma_{D}$ & \multicolumn{4}{|c|}{0.7} \\
\hline Noise amplification $\alpha$ & \multicolumn{4}{|c|}{1.5} \\
\hline Nbr. pixels $N_{X}$ & \multicolumn{4}{|c|}{4} \\
\hline Seeing at $0.5 \mu \mathrm{m}$ & \multicolumn{4}{|c|}{0.5 arcsecond } \\
\hline $\begin{array}{l}\text { Coherence time } \\
\text { at } 0.5 \mu \mathrm{m}\end{array}$ & \multicolumn{4}{|c|}{$7 \mathrm{~ms}$} \\
\hline Atmospheric residual $\mathrm{T}$ & \multicolumn{4}{|c|}{200} \\
\hline $\begin{array}{l}\text { Isoplanatic angle } \\
\text { at } 0.5 \mu \mathrm{m}\end{array}$ & \multicolumn{4}{|c|}{2.6 arcsecond } \\
\hline Outer Scale & \multicolumn{4}{|c|}{$25 \mathrm{~m}$} \\
\hline Nbr. pairs $N_{P}$ & 3 & 3 & 3 & 1 \\
\hline $\begin{array}{l}\text { Nbr. spectral } \\
\text { channels } N_{\lambda}\end{array}$ & 6 & 6 & 6 & 1 \\
\hline $\begin{array}{l}\text { Spectral band } \\
\text { phase delay }\end{array}$ & $K$ & $K$ & $K$ & $1.58-2.3 \mu \mathrm{m}$ \\
\hline $\begin{array}{l}\text { Spectral band } \\
\text { group delay }\end{array}$ & $K$ & $K$ & $K$ & $1.1-1.58 \mu \mathrm{m}$ \\
\hline Transmission & 1 percent & 1 percent & 1 percent & $\begin{array}{l}2 \text { per cent } \\
2.9 \times 10^{9} \\
(\mathcal{G} \text { Delay })\end{array}$ \\
\hline Photons/s $\left(K=0, S_{t r}=1\right)$ & $6 \times 10^{7}$ & $1.2 \times 10^{9}$ & $1.2 \times 10^{9}$ & $\begin{array}{l}4.4 \times 10^{9} \\
\left(\varphi_{\text {Delay }}\right)\end{array}$ \\
\hline Strehl in $K$ & 0.5 & & (LGS mod & \\
\hline Strehl in $H$ & - & - & - & 0.4 \\
\hline Strehl in $J$ & - & - & - & 0.2 \\
\hline $\begin{array}{l}\text { Vibration residual } \sigma_{V} \\
\qquad\left(\pi^{2}\left(\sigma_{V}^{2}+\sigma_{T}^{2}\right)\right. \\
-1\end{array}$ & 100 & 200 & 100 & 100 \\
\hline$V_{I}=\exp \left(-\frac{v}{2 \lambda^{2}}\right)$ & 0.81 & 0.69 & 0.81 & 0.75 \\
\hline$V_{E}=\sqrt{1-\gamma^{2}}$ & $\sim 1$ & $\sim 0.16$ & $\sim 1$ & $\sim 1$ \\
\hline Baseline & $\mathrm{G}_{1}-\mathrm{J}_{3}$ & $\begin{array}{l}\mathrm{UT}_{1}- \\
\mathrm{UT}_{4} \\
\end{array}$ & $\begin{array}{l}\mathrm{UT}_{1-} \\
\mathrm{UT}_{4} \\
\end{array}$ & $\begin{array}{l}\mathrm{UT}_{1-} \\
\mathrm{UT}_{4}\end{array}$ \\
\hline
\end{tabular}

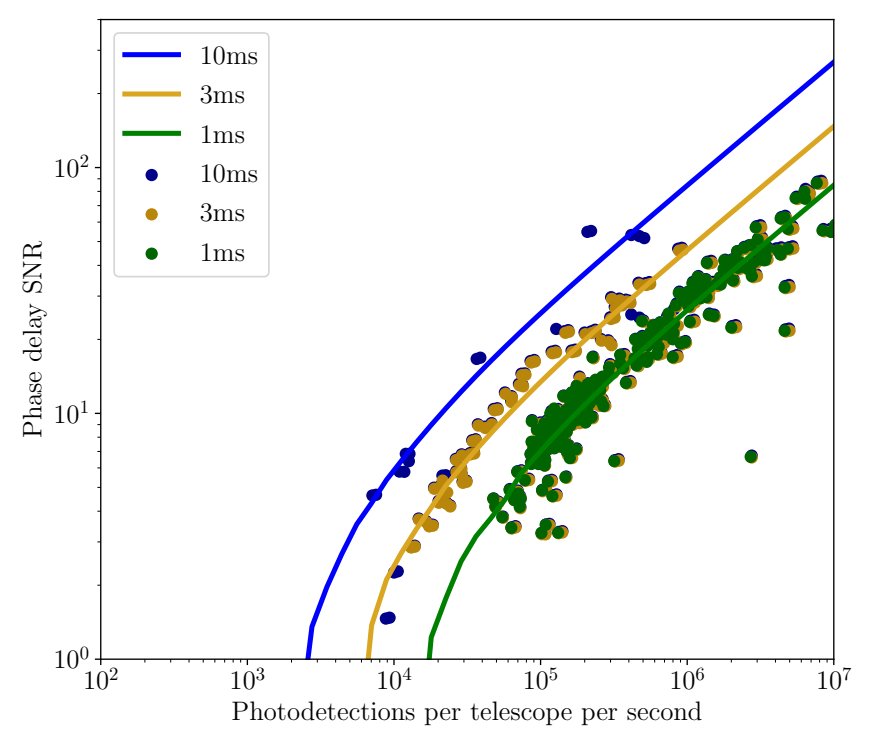

Figure 5. Phase delay as function of the number of detected photons per telescope per second. The full lines are for frame times $1 \mathrm{~ms}$ (green), $3 \mathrm{~ms}$ (gold), and $10 \mathrm{~ms}$ (blue). The dots represent measured SNR values with the GFT copied from (Lacour et al. 2019). 
maximize $V_{i j}$ and $\sqrt{1-\gamma^{2}}$. Minimizing the interferometer vibrations will increase $V_{i j}$. An optimization of the AO, from the telescope to the control of image motion in the focal laboratory, will increase $\sqrt{1-\gamma^{2}}$. Then, we have to use the longest possible frame time $\mathcal{T}_{F}$ that is limited by the atmospheric turbulence as discussed in detail in section 2.10 but also by the vibrations of the interferometer. To maximize the collected flux $n_{*}$, we can increase the Strehl ratio of the AO and the transmission of the instrument. Increasing the transmission of the interferometer itself is quite difficult in an existing interferometer but will be a key design parameter in any new generation interferometer such as PFI. We can also maximize the wavelength range used for phase delay tracking but in doing so we have to avoid increasing too much the background photon noise $n_{B}$ that grows very rapidly with wavelength after $2.3 \mu \mathrm{m}$. Finally, we can work on the phase delay tracker itself, by reducing the triplet $N_{X}, N_{\lambda}$, and $N_{P}$, where $N_{X}$ is the number of pixels necessary to measure a phase delay, $N_{\lambda}$ is the number of spectral channels, and $N_{P}$ is the numbers of the unit phase delay sensors fed by each telescope.

Maximizing the AO efficiency and minimizing the vibrations of the VLTI are two key elements of the GRAVITY+ plan. They would also be corner stones of any new interferometer such as PFI together with an optimization of its global transmission. In this paper, we focus on the optimization of the phase delay sensor that is described in the next section.

\subsection{Fringe sensing optimization}

Historically, the first alternative to pairwise FTs has been based on 'global' beam combiners like the ones used in Petrov et al. (2007) and Lopez et al. (2014). For such a system, equation (17) becomes:

$$
\begin{aligned}
Z_{i j G} & =\frac{\sqrt{\operatorname{Var}\left(\mathfrak{J} C_{i j G}\right)}}{\left\langle\mathfrak{R} C_{i j G}\right\rangle} \\
& =\frac{\sqrt{N_{T}\left(n_{*}+n_{B}\right) \mathcal{T}_{F} \alpha^{2}+N_{X G} N_{\lambda} \sigma_{R}^{2}}}{\sqrt{2} \mathcal{T}_{F} n_{*} V_{i j} \sqrt{1-\gamma_{i j}^{2}}} .
\end{aligned}
$$

$N_{T}$ is the number of telescopes and $N_{X G}$ is the number of pixels necessary to sample a global interferogram. The minimum value for $N_{X G}$ is set by the number of baselines:

$N_{X G}=2 \mathcal{K} N_{T}\left(N_{T}-1\right)$,

the term $\mathcal{K}$ depends on the specifications of the beam combiner. To measure properly all scientific measurable of an interferometer, we need $\mathcal{K}>2$ and for example in MATISSE $\mathcal{K}>6$. For a system designed only to measure only phase delays in a fringe sensor, it is possible to set $\mathcal{K}=2$, at least to be able to use the standard data processing routines with fully evaluated noise propagation. Blind et al. (2011) claims that pairwise FT is better when the noise is dominated by detector read-out noise and Lopez et al. (2014) show that global beam combiners are better when the noise is dominated by background noise. Comparing equations (17 and 35) at the sensitivity limit; when noise is dominated by the detector read-out yields:

$$
\left(\frac{Z_{i j G}}{Z_{i j L}}\right)^{2}=\frac{\mathcal{K} N_{T}}{2\left(N_{T}-1\right)}<1 \text { if } \mathcal{K}<\frac{2\left(N_{T}-1\right)}{N_{T}} .
$$

This confirms an advantage to pairwise systems for $N_{T}=4$ and $\mathcal{K} \geq 2$ when all baselines are used in the same way.

Many authors (e.g. Meisner et al. 2012; Petrov et al. 2016; Blind et al. 2011) have proposed systems where only a fraction of the $\frac{N_{T}\left(N_{T}-1\right)}{2}$ baselines are used to evaluate the $N_{T}-1$ phase delays, i.e. to reduce $N_{P}$ that is the number of pairwise unit sensors fed by a telescope. The ultimate solution seems to be the HFT that reduces $N_{P}$ to the minimum value of 1 .

\subsection{The HFT phase delay sensor}

The HFT (Petrov et al. 2014, 2016) splits the phase delay measurements between different levels. In the first level, we cophase pairs of apertures individually using all the available flux $\left(N_{P}=1\right)$. Then we transmit the cophased flux to the next level as if it was produced by a single cophased aperture. If the transmission of the first level is larger than 50 per cent, the second level of the HFT that cophases pairs of pairs of telescopes, is fed by more photons than the first level and it has a higher sensitivity if the source is unresolved on all baselines, which is always the case with the VLTI for the very faint fringe tracking targets considered in this paper. Then the cophased beams are again fed in the next level that cophases pairs of groups of telescopes and so on. As the phase delay sensing SNR increases in an HFT with the hierarchical level, the limiting performances are set by the first level that cophases two telescopes.

An additional characteristic of the HFT is that the beam combination and the phase delay estimation at each level are performed by a modified $\mathrm{ABCD}$. In an ABCD beam combiner, we have four outputs corresponding to $0, \pi / 2, \pi$, and $-\pi / 2$ (or the equivalent $3 \pi / 4,-\pi / 4, \pi / 4$, and $3 \pi / 4$ ) phase delays between the two input beams. The maximum flux in one of these outputs is 47.5 per cent of the total input (without transmission losses). In the HFT, we use a beam combiner with three outputs at $\mathrm{A}:-3 \pi / 4, \mathrm{C}: 0$, and $\mathrm{B}: 3 \pi / 4$. The central output is obtained by merging the $-\pi / 4$ and $\pi / 4$ outputs of an $\mathrm{ABCD}$ after introducing the appropriate fixed phase shift that cophases them whatever the piston between the two input beams, as illustrated in Fig. 6 .

The estimator $\phi=\arg (A-B, C-A-B)$ yields the phase delay between the input beams independently of the source visibility and flux ratio between input beams (Petrov et al. 2016). When the two input beams are cophased and have the same intensity, the central output of this ACB beam combiner contains 80.8 per cent of the total input (without transmission losses). This beam can then be efficiently fed in an identical ACB beam combiner of the $2^{\text {nd }}$ level of the HFT as illustrated by Fig. 7 .

Fig. 6 and 7 that illustrate the conceptual design of a four telescopes HFT also show the design of integrated optics components of an HFT that have been manufactured and successfully tested. There are also bulk optic solutions to implement these concepts. They can yield a much better transmission but have a more complex optomechanical design and a loss in precision resulting from the lack of the perfect spatial filtering introduced by single mode fibers or integrated optics. However, this should have a limited impact on the phase delay accuracy, at least for the moderate tracking quality discussed here. We leave the discussion of this point for future work and in this paper we use only transmissions compatible with single mode filtering. The full description of the HFT and of its proof of concept from analytical computations, numerical simulations, and laboratory tests will be described in another paper (Boskri et al., in preparation). Here, we just have to note that the phase delay SNR of an HFT is set by equation (17) with $N_{P}=1$ and $N_{X}=4$.

For a maximum efficiency of the HFT phase delay measurements, we also decided to use very broad-band measurements with $N_{\lambda}$. Equation (17) then becomes for the first level of the HFT:

$Z_{i j H 1}=\frac{\sqrt{\left(n_{*}+n_{B}\right) \mathcal{T}_{F} \alpha^{2}+1.5 \sigma_{R}^{2}}}{\mathcal{T}_{F} n_{*} V_{i j} \sqrt{1-\gamma_{i j}^{2}}}$.

As our goal in this paper is to evaluate the sky coverage for the best fringe sensor that can be reasonably foreseen, we will also consider 

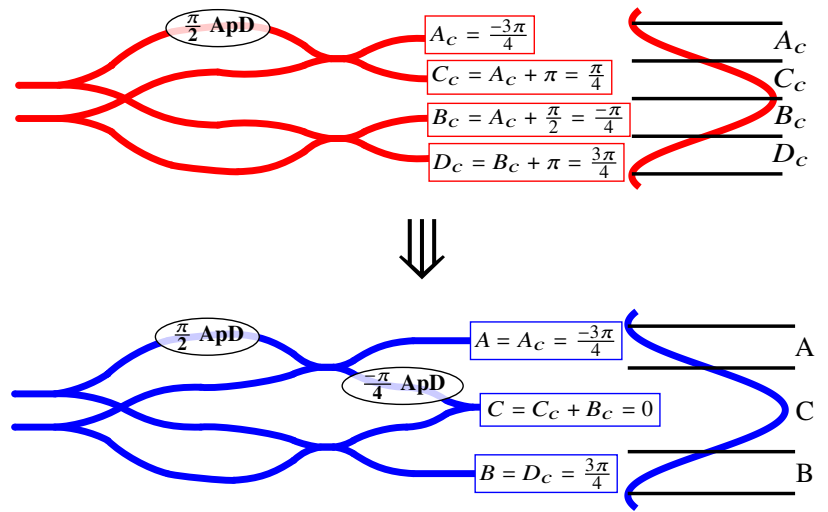

$A p D$ : Achromatic Phase Delay

Figure 6. Conceptual design of the ACB beam combiner that is the basic unit of a HFT. The upper image shows the classical ABCD beam combiner used in PIONIER or GRAVITY with its four outputs at $-\pi / 2, \pi / 2,0$, and $-\pi$. The bottom image shows our modified beam combiner where the ' $\pi / 2$ ' and ' 0 ' outputs of the ABCD have been cophased by an appropriate phase shift and then merged.

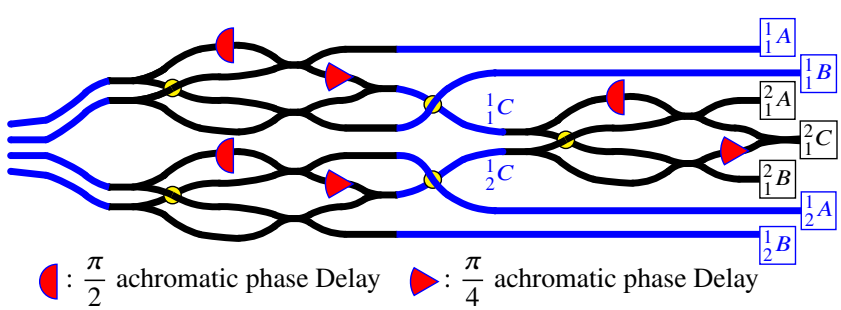

Figure 7. Conceptual design of a four telescopes HFT, with two levels. Note that in this design the central outputs ${ }_{1}^{1} C$, and ${ }_{2}^{1} C$, of the first level are not measured directly but deduced from the seven outputs of the system.

that the HFT uses 80 per cent of the full $H$ and $K$ spectral bands, which is acceptable for off-axis tracking where we do not need to feed a fraction of this bands into science instruments that work on-axis. We eliminate the wavelengths larger than $2.3 \mu \mathrm{m}$ because their contribution to SNR loss due to background noise exceeds the gain provided by their contribution to the source flux. We also reserve a fraction of the $\mathrm{H}$ band for the group delay sensor discussed in the next section.

Finally, we estimate that the overall efficiency of a new generation FT will be 2 per cent instead of the current 1 per cent value for the GFT. Beyond the gain provided by the fact that we do not include dispersive elements in our design, this 2 per cent value is quite conservative, as for example the overall transmission of the AMBER VLTI instrument, with its complex combination of internal dichroic plates to split and then recombine the $J, H$, and $K$ bands; optical fibers for spatial filtering and the high-resolution spectrograph, was of the order of 4 per cent in the $K$ band.

The final numbers used in this paper to estimate the sensitivity and the sky-coverage with an HFT are given in Table 1. Note that we take into account the variation of Strehl ratio and instrument visibility as a function of wavelength as well as the average magnitude difference $\mathrm{H}-\mathrm{K}=0.1$ and $\mathrm{J}-\mathrm{K}=0.5$ for our(very large) selection of calibrators. The corresponding HFT phase delay SNR, computed from equation (38) combined with equation (23), is displayed in Fig. 8.

\subsection{Group delay sensing in association with the HFT}

With $N_{\lambda}=1$, the HFT sketched above cannot control the group delay. As it is a very broadband system, the phase delay SNR and the global transmission in the central ' $\mathrm{C}$ ' outputs will have a strong maximum at

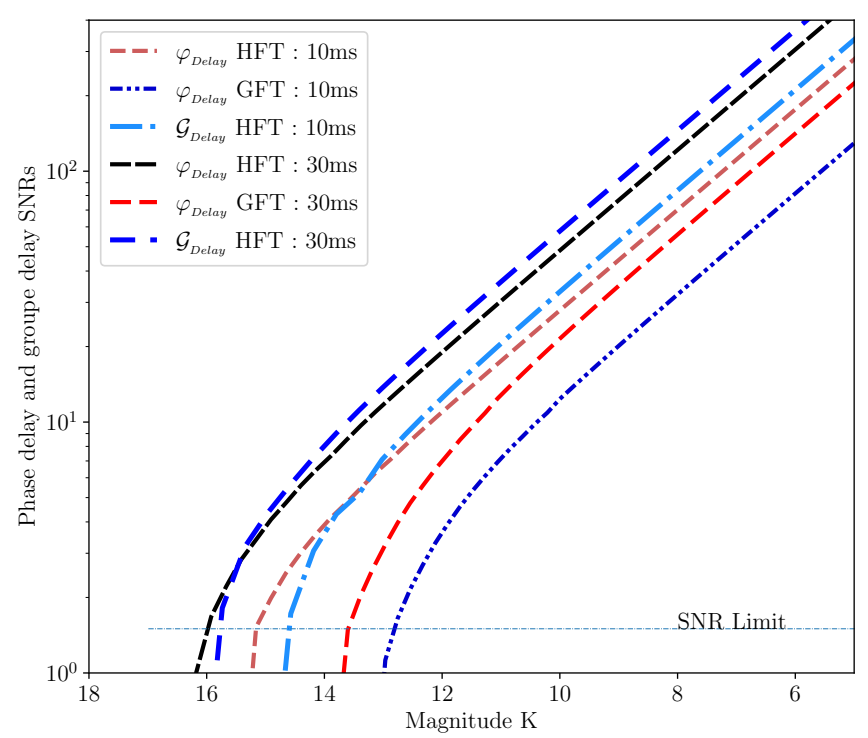

Figure 8. Phase delay and group delay SNR as a function of the $K$ band magnitude for the HFT and GFT used with UTs with the future GRAVITY+ AO.

$\mathrm{OPD}=0$ allowing to detect immediately that the central fringe has been lost, but we would not know in what direction to search to reacquire it and scan the baselines for that purpose is considered as too costly in observing time.

There are several options to associate a group delay sensor to the HFT. The original plan was to install a group delay sensor in the final output of the HFT (labelled ${ }_{1}^{2} C$ in Fig. 7). This works in principle but implies some design and data processing complexity. Another option is to use the $\mathrm{J}$ band and a small fraction of the $\mathrm{H}$ band to implement a classical group delay sensor based on the same dispersed fringe scheme as MATISSE. This yields the parameters in Table 1 for the group delay estimation that is plotted in Fig. 8 for a $1 \mathrm{~Hz}$ Group Delay frequency. Another possibility is to disperse the A and B outputs of the HFT over 3 spectral channels covering the $J, H$, and $K$ bands with a minor degradation of the phase delay performance. The phase delay SNR of such a $N_{\lambda}=3$ JHK HFT is also plotted in Fig. 8.

\subsection{Anisopistonic variance}

The anisopistonic variance $\sigma_{A}^{2}(\theta)$ is the variance of the difference between the piston observed in two different directions with angular separation $\theta$. Elhalkouj et al. (2008) developed and tested an analytical expression of $\sigma_{A}^{2}(\theta)$ as a function of $\mathrm{D}$ and the seeing parameters $r_{0}$, $L_{0}$ and the isoplanatic angle $\theta_{0}$. Here, we summarize the key steps and parameters of that analysis for the commodity of the reader.

Fig. 9 shows the general geometry of the problem. The variance $\sigma_{A}^{2}$ of the differential piston difference is:

$$
\begin{aligned}
\sigma_{A}^{2} & =\left\langle\left[\left(P_{2}-P_{1}\right)-\left(P_{2}^{\prime}-P_{1}^{\prime}\right)\right]^{2}\right\rangle \\
& =4\left\langle P_{1}^{2}\right\rangle-4\left\langle P_{1} P_{2}\right\rangle+2\left\langle P_{1} P_{1}^{\prime}\right\rangle+2\left\langle P_{2} P_{1}^{\prime}\right\rangle+2\left\langle P_{1} P_{2}^{\prime}\right\rangle
\end{aligned}
$$

where $P_{i}$ and $P_{i}^{\prime}$ are the average OPD of the layer over the pupil $i$ observed in two directions separated by an angle $\theta$.

Assuming $\left\langle P_{1}^{2}\right\rangle=\left\langle P_{2}^{2}\right\rangle, \quad\left\langle P_{1} P_{2}\right\rangle=\left\langle P_{1}^{\prime} P_{2}^{\prime}\right\rangle$, and $\left\langle P_{1} P_{1}^{\prime}\right\rangle=$ $\left\langle P_{2} P_{2}^{\prime}\right\rangle$ for a homogeneous and isotropic turbulence. Considering that $\Delta \gg h_{m} \theta$, we can write $d_{12} \simeq d_{21} \simeq \Delta$ and $\left\langle P_{1} P_{2}\right\rangle \simeq\left\langle P_{1} P_{2}^{\prime}\right\rangle \simeq$ 


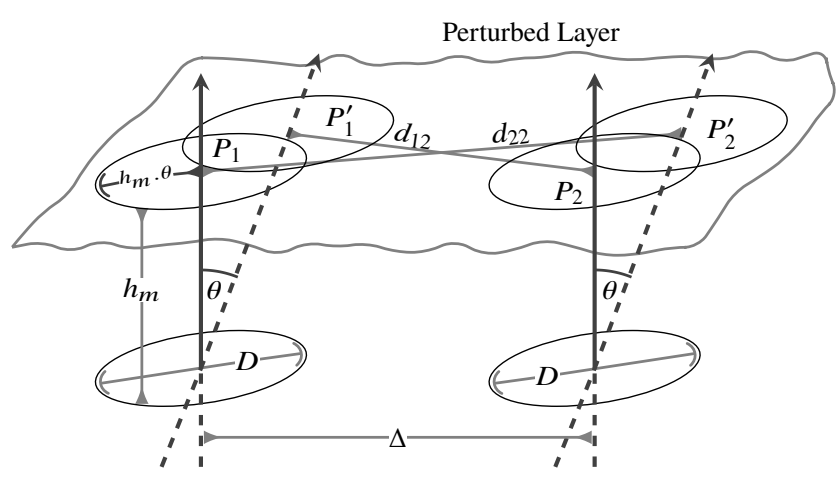

Figure 9. The geometrical parameters to compute the contribution of a turbulent layer at altitude $h_{m}$ to the difference of piston between two directions separated by $\theta . D$ is the telescope diameters and $\Delta$ is the baseline.

$\left\langle P_{2} P_{1}^{\prime}\right\rangle$ and hence:

$\sigma_{A}^{2}=4\left\langle P_{1}^{2}\right\rangle+2\left\langle P_{1} P_{1}^{\prime}\right\rangle$.

The variance $\left\langle P_{1}^{2}\right\rangle$ and the covariance $\left\langle P_{1} P_{1}^{\prime}\right\rangle$ are computed from the integration of OPD differences over the pupil of diameter D. Assuming a Von Karman model for the structure function of the phase, Takato \& Yamaguchi (1995) write:

$$
\begin{aligned}
\sigma_{A}^{2}(\theta) & =0.02294 \pi^{2 / 3} \lambda^{2}\left(\frac{D}{r_{0}\left(h_{m}\right)}\right)^{5 / 3} \\
& \times \int_{0}^{\infty} \frac{2 J_{1}^{2}(x)}{x\left[x^{2}+\left(\pi \frac{D}{L_{0}\left(h_{m}\right)}\right)^{2}\right]^{11 / 6}}\left[1-J_{0}\left(2 x \frac{h_{m} \theta}{D}\right)\right] \mathrm{d} x,
\end{aligned}
$$

where $r_{0}\left(h_{m}\right)$ and $L_{0}\left(h_{m}\right)$ are the Fried parameter and the outer scale for the turbulence in the layer at altitude $h_{m}$. For each layer of thickness $\Delta h$, we have:

$\lambda^{2} r_{0}\left(h_{m}\right)^{-5 / 3}=\frac{C_{N}^{2}\left(h_{m}\right) \Delta h}{0.06}$.

In the standard near-field approximation, the overall OPD variance is the sum of the single layer variances and putting equation (42) in (41) yields:

$$
\begin{aligned}
\sigma_{A}^{2}(\theta) & =3 \pi^{\frac{2}{3}} D^{\frac{5}{3}} \int_{0}^{\infty} \int_{0}^{\infty} \frac{2 J_{1}^{2}(x) C_{N}^{2}(h)}{x\left[x^{2}+\left(\pi \frac{D}{L_{0}\left(h_{m}\right)}\right)^{2}\right]^{\frac{11}{6}}} \\
& \times\left[1-J_{0}\left(2 x \frac{h_{m} \theta}{D}\right)\right] \mathrm{d} x \mathrm{~d} h .
\end{aligned}
$$

Elhalkouj et al. (2008) has developed an analytical expression of equation (43) based on a development of the integral in convergent series using a technique based on the convolution theorem of the Mellin transform introduced by Sasiela (1994). This development yields:

$\sigma_{A}^{2}(\theta)=\pi^{\frac{2}{3}} \lambda^{2}\left(\frac{D}{r_{0}}\right)^{-1 / 3}\left(\frac{\theta}{\theta_{0}}\right)^{2} P_{A}\left(\frac{\pi D}{L_{0}}\right)$,

where we have introduced the isoplanatic angle $\theta_{0}$ from Fried (1982):

$\theta_{0}=0.058 \lambda^{6 / 5}\left[\int_{0}^{\infty} h^{5 / 3} C_{N}^{2}(h) \mathrm{d} h\right]^{-3 / 5}$,

$P_{A}$ is a polynomial function with parameters dominated by the ratio between the telescope diameter and the outer scale $\frac{\pi D}{L_{0}}$. Elhalkouj et al.

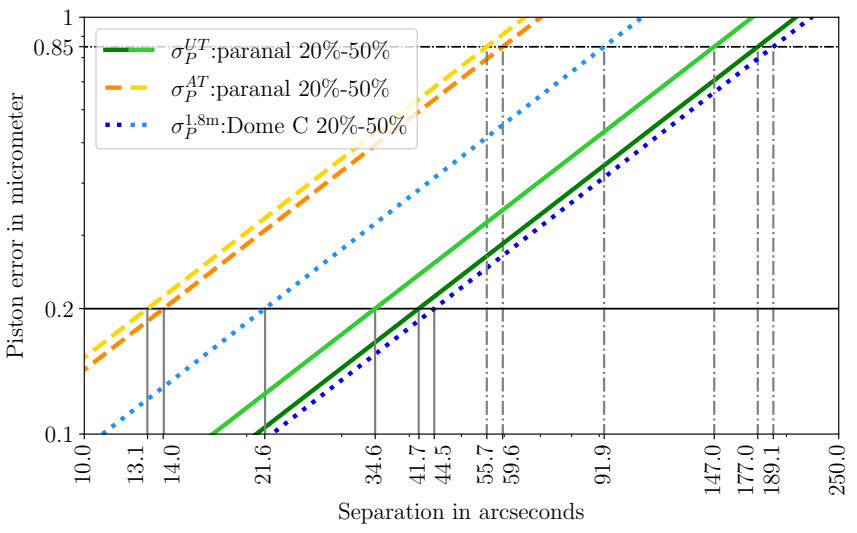

Figure 10. Anisopistonic error as a function of the separation between the observed target and the off-axis star used for fringe tracking in logarithmic scale. In dashed lines, yellowish with $8 \mathrm{~m}$ telescopes (UTS) at Paranal in the best 20 per cent seeing (upper curve) and in median seeing (lower curve). In full-line greenish with $1.8 \mathrm{~m}$ telescopes (ATs) at Paranal, for best 20 per cent seeing (upper curve) and median seeing (lower curve). In dotted line blueish, at Dome $\mathrm{C}$ for $1.8 \mathrm{~m}$ telescopes placed above the $30 \mathrm{~m}$ ground layer. The list of numbers below the abscissa axis is the Isopistonic angle.

(2008) defines a small aperture regime by $D<\frac{\alpha L_{0}}{\pi}$ where:

$$
\begin{aligned}
P_{A}\left(\frac{\pi D}{L_{0}}\right) & =8.8410^{-2}\left[0.216-0.225\left(\frac{\pi D}{L_{0}}\right)^{\frac{1}{3}}\right. \\
& +0.122\left(\frac{\pi D}{L_{0}}\right)^{2}-0.096\left(\frac{\pi D}{L_{0}}\right)^{\frac{7}{3}}+0.02\left(\frac{\pi D}{L_{0}}\right)^{4} \\
& \left.-0.014\left(\frac{\pi D}{L_{0}}\right)^{\frac{13}{3}}\right] .
\end{aligned}
$$

For $\alpha<1.3$, the difference between the results of equation (46) and equation (43) are smaller than 5 per cent and they reach 10 per cent for $\alpha \sim 2.6$, this allows using equation (46) for all the cases considered in this paper. In equation (46), almost all the sensitivity of $\sigma_{A}^{2}$ to the exact vertical distribution of turbulence described by the profile $C_{n}^{2}(h)$ is included in the terms $r_{0}, L_{0}$, and $\theta_{0}$ evaluated on the ground. However, very special turbulent profiles such as a very strongly dominant layer (near the ground or at the troposphere) might introduce deviations.

Fig. 10 gives the anisopistonic error computed from equation (46) for the three cases considered here. UTs and ATs at Paranal, in the median seeing conditions $\left(L_{0}=16 \mathrm{~m}, r_{0}=0.76 \mathrm{~m}\right)$ and best 20 per cent seeing condition $\left(L_{0}=22 \mathrm{~m}, r_{0}=0.53 \mathrm{~m}\right)$ and $1.8 \mathrm{~m}$ telescopes at the Dome $\mathrm{C}$. It allows reading the values of the isopistonic angle $\theta_{P}(\lambda=2 \mu m)$ defined by $\sigma_{A}\left[\theta_{P}(\lambda)\right]=\frac{\lambda}{10}$ that are given in Table 2 .

Note the very strong dependence of the isopistonic angle on the telescope diameter. The isopistonic angle is very large and the maximum separation between the observed target and the guide star are more likely to be limited by the hardware at the telescope or by AO isoplanetism than by the isopistonic angle in the $K$ band. Equation (44) and Fig. 9 gives the anisopistonic error for a target at zenith. To compute its value at a zenith distance $z$, we use the dependence of $r_{0}$ and $\theta_{0}$ with that distance:

$\left\{\begin{array}{l}r_{0}(z)=r_{0}(0)(\cos z)^{3 / 5} \\ \theta_{0}(z)=\theta_{0}(0) \cos (z)\end{array}\right.$

The outer scale $L_{0}$ is a parameter describing the turbulence "where it is' that does not change with the zenith distance. 
Table 2. Isopistonic angle, in different sites and seeing conditions, for a $\lambda / 10$ error at $\lambda=2 \mu \mathrm{m}$. The isopistonic angle is proportional to the wavelength.

\begin{tabular}{llll}
\hline & $\begin{array}{l}\mathrm{D}=8 \mathrm{~m}, \\
\text { Paranal }\end{array}$ & $\begin{array}{l}\mathrm{D}=1.8 \mathrm{~m}, \\
\text { Paranal }\end{array}$ & $\begin{array}{l}\mathrm{D}=1.8 \mathrm{~m}, \\
\text { Dome C }\end{array}$ \\
\hline $\begin{array}{l}\text { Best 20 per cent } \\
\text { seeing }\end{array}$ & 41.7 arcsecond & 14.0 arcsecond & 44.5 arcsecond \\
Median seeing & 34.6 arcsecond & 13.1 arcsecond & 21.6 arcsecond \\
\hline
\end{tabular}

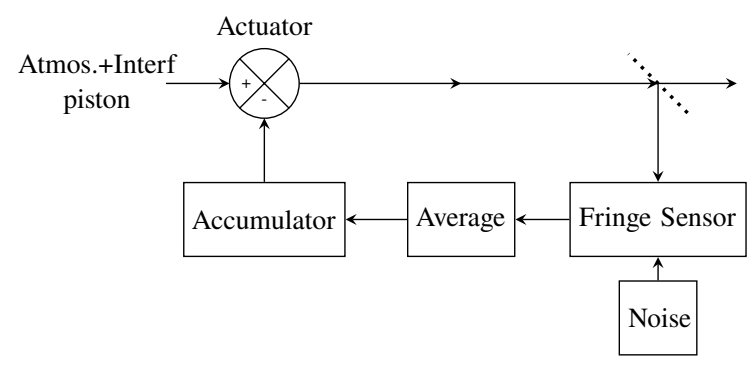

Figure 11. The simple servo loop of FTs used in this paper.

\subsection{Servo loop variance}

In this paper, we will consider only the simplest servo loop that is sketched in Fig. 11. The fringe sensor measures the phase delay introduced by the atmosphere and the interferometer after the last piston correction applied by the piezo actuator. The measure is affected by the detection noise discussed in the previous section. The resulting error signal is averaged over a dwell time $\mathcal{T}_{F}$ and subsequently passed to an accumulator that adds the measured error to the previous piston actuator position.

Actual FTs, such as the GFT, use more sophisticated phase delay controllers based on a state-space auto-regressive representation of the atmospheric and vibration perturbation with a Kalman filter optimization. Here we consider that such a device only allows approaching the performances of the simple integrator affected only by the atmospheric piston that we analyze in the following. This might be a fair or even quite optimistic representation of the current status of the GFT on UTS that fails to reach the longest frame times allowed by the atmosphere. It is probably a slightly pessimistic description of a GRAVITY+ optimum situation where vibrations and injection instabilities have been damped below the effects of the atmospheric piston by systems independent from the FT. The simple loop used here has the great advantage to allow an analytical expression of the effects of the atmospheric piston.

Frequency-domain models for each of the components of the loop shown in Fig. 11 can be found in many papers, e.g. Femenia et al. (2000). If we note $\underline{Y}_{i n}(f)$ and $\underline{Y}_{\text {out }}(f)$, the input and output signal of the servo loop system in the frequency domain, the succession of elements in Fig. 11 translates into the following equation :

$$
\underline{Y}_{\text {out }}(f)=\underline{Y}_{\text {in }}(f)-\underline{H}_{p z}(f) \underline{H}_{S}(f) \underline{H}_{a}(f) \times \underline{Y}_{\text {out }}(f) .
$$

This gives the square modulus of the servo loop error-input transfer function

$$
H_{\text {loop }}(f)=\left|\frac{1}{1+\underline{H}_{p z}(f) \underline{H}_{S}(f) \underline{H}_{a}(f)}\right|^{2} .
$$

The fringe sensor (sampling and averaging) system includes an integration period $\mathcal{T}_{F}$ and an average delay time of $\mathcal{T}_{L}$ between the input and the output signals. If the computation time is negligible, we have $\mathcal{T}_{L} \simeq \mathcal{T}_{F} / 2$. Then the transfer function for the fringe sensor is given by:

$$
\underline{H}_{S}(f)=\frac{\sin \left(\pi f \mathcal{T}_{F}\right)}{j \pi f \mathcal{T}_{F}} \exp \left(-j \pi f \mathcal{T}_{F}\right)=\frac{1-\exp \left(-j 2 \pi f \mathcal{T}_{F}\right)}{2 j \pi f \mathcal{T}_{F}} .
$$

The accumulator adds the incremental error signal to the current piezo-mirror position. This is represented by the function

$$
\underline{H}_{a}(f)=\frac{1}{2 j \pi f \mathcal{T}_{F}} .
$$

The piezo-actuator system is generally very fast and has a nearly instantaneous response, yielding $\underline{H}_{p z}=1$, and hence:

$$
\begin{aligned}
H_{\text {loop }}(f) & =\left|\frac{1}{1+\underline{H}_{s}(f) \underline{H}_{a}(f)}\right|^{2} \\
& =\frac{\left(2 \pi f \mathcal{T}_{F}\right)^{4}}{\left(2 \pi f \mathcal{T}_{F}\right)^{4}-4 \sin ^{2}\left(\pi f \mathcal{T}_{F}\right)\left[\left(2 \pi f \mathcal{T}_{F}\right)^{2}-1\right]}
\end{aligned}
$$

This last expression is approximated by Parenti \& Sasiela (1994) as:

$$
H_{\text {loop }}(f) \simeq \frac{\left(2 \pi f \mathcal{T}_{F}\right)^{2}+\frac{4}{89}\left(2 \pi f \mathcal{T}_{F}\right)^{4}}{1+\frac{2}{45}\left(2 \pi f \mathcal{T}_{F}\right)^{4}},
$$

and the residual piston variance introduced in the servo loop system by the change of the atmospheric piston through the loop cycle is then given by:

$$
\sigma_{L}^{2}=\left(\frac{\lambda}{2 \pi}\right)^{2} 2 \int_{0}^{\infty} H_{\text {loop }}(f) W_{\phi}(f) \mathrm{d} f
$$

where $W_{\phi}(f)$ is the power spectrum of the piston phase $\phi=2 \pi p_{i j} / \lambda$. That power spectrum and that integral have been computed by Femenia et al. (2000) assuming a Von Karman spectrum with outer scale $L_{0}$ for the random atmospheric refractive index fluctuations, which were supposed to be isotropic within each turbulent layer. The temporal variations of the piston are computed using a multilayer Taylor model, with turbulent layers drifting with the wind through the telescope aperture faster than they change internally. It writes:

$\sigma_{L}^{2}=A_{0} \sec (z) D^{-1 / 3} \mathcal{T}_{F}^{2} v^{2}\left[1+A_{1}\left(D, L_{0}\right)+A_{2}\left(D, L_{0}, \Delta\right)\right]$,

where $z$ is the zenith distance and $v^{2}=\int_{0}^{\infty} C_{N}^{2}(h) v^{2}(h) \mathrm{d} h$ is the $2^{\text {nd }}$ order moment of the velocity components in the direction of the interferometric baseline that provides an upper limit for $W_{\phi}(f)$.

The parameters $A_{1}, A_{2}$, and $A_{3}$ have different expressions in the different regimes set by the relative size of the outer scale $L_{0}$ and of the telescope diameter $D$. In all cases considered here, the telescope diameter is much smaller than turbulence outer scale and then Femenia et al. (2000) derives the following values of $A_{0}, A_{1}$, and $A_{2}$ :

$$
\left\{\begin{array}{rl}
A_{0}= & 0.643 \\
A_{1}= & 3.61 \times 10^{-2}\left(L_{0} / D\right)^{8 / 3} \times \\
& { }_{2} F_{1}\left(4 / 3,3 / 2 ; 5 / 2 ;-0.09\left(L_{0} / D\right)^{2}\right) \\
A_{2}= & -1.07 \times 10^{-2}\left(L_{0} / D\right)^{8 / 3}\left(1-0.474(D / \Delta)^{1 / 3}\right) \times \\
& { }_{2} F_{1}\left(4 / 3,3 / 2 ; 5 / 2 ;-0.09\left(L_{0} / D\right)^{2}\right)
\end{array},\right.
$$

where $\Gamma(x)$ is the gamma function and ${ }_{2} F_{1}$ is the Gauss hypergeometric function given by Sasiela (1994):

${ }_{2} F_{1}[\alpha, \beta ; \gamma ; z]=\sum_{k=0}^{\infty} \frac{(\alpha)_{k}(\beta)_{k}}{(\gamma)_{k}} \frac{z^{k}}{k !} ;$ where $(a)_{k}=\frac{\Gamma(a+k)}{\Gamma(a)}$

Introducing the average velocity

$$
\bar{v}_{2}=\left[\frac{\int_{0}^{\infty} C_{N}^{2}(h) v^{2}(h) \mathrm{d} h}{\int_{0}^{\infty} C_{N}^{2}(h) \mathrm{d} h}\right]^{1 / 2}
$$

and the Fried parameter $r_{0}$ defined by $\int_{0}^{\infty} C_{N}^{2}(h) \mathrm{d} h=0.06 \lambda^{2} r_{0}^{-5 / 3}$, we can write

$$
\sigma_{L}^{2} \simeq 0.06 A_{0} \sec (z) \lambda^{2} r_{0}^{-5 / 3} D^{-1 / 3} \mathcal{T}_{F}^{2}\left(\bar{v}_{2}\right)^{2}\left[1+A_{1}+A_{2}\right],
$$




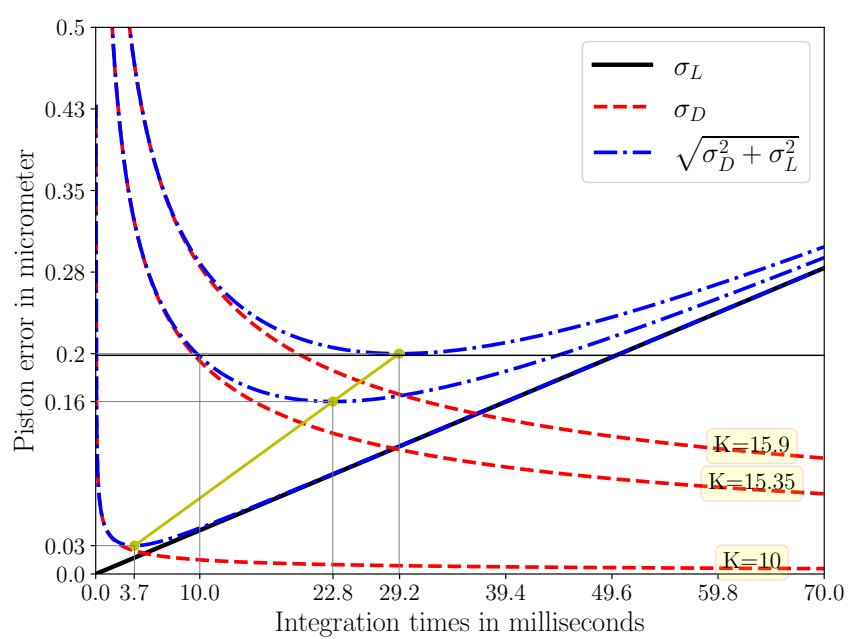

(a) HFT-UT Paranal

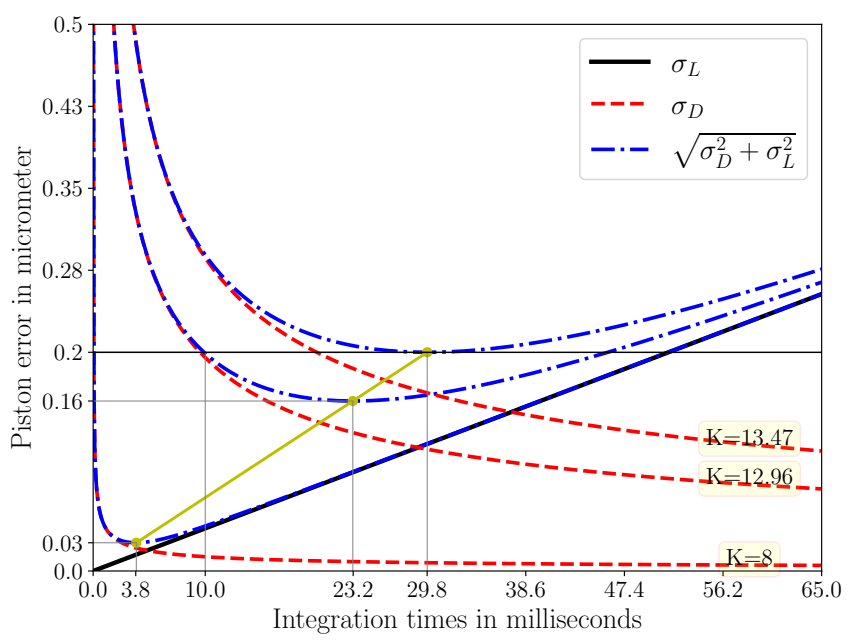

(b) GFT-UT Paranal

Figure 12. Tracking error as a function of the frame time, for various magnitudes $K$ (blue dot-dashed curves). The black line is the loop error and the red dashed lines are detection errors. The optimum frame times, which minimize the tracking error for a given magnitude, are indicated in green.

which can be expressed in terms of the atmospheric coherence time $\mathcal{T}_{0}$ as the following:

$$
\sigma_{L}^{2}=5.7610^{-3} A_{0} \sec (z) \lambda^{2}\left(\frac{r_{0}}{D}\right)^{1 / 3} \frac{\mathcal{T}_{F}^{2}}{\mathcal{T}_{0}^{2}}\left[1+A_{1}+A_{2}\right],
$$

As all parameters in equation 58 are constant for given seeing conditions, at least when $\mathcal{T}_{F} \ll L_{0} / \bar{v}_{2}$ i.e. the time needed for an outer scale to be drifted away. Then the loop error introduced by the atmospheric piston is proportional to the fringe sensor dwell time $\mathcal{T}_{F}$.

\subsection{Tracking error on the guide star and optimum frame time}

For the source used for fringe sensing and tracking, we will have a residual piston error with variance $\sigma_{F}^{2}=\sigma_{D}^{2}+\sigma_{L}^{2}$ if we assume that the fringe sensing error and the loop error are independent random variables. We have seen that $\sigma_{D}^{2}$ is a function of $\sigma_{L}^{2}$ but in a stationary tracking regime the tracking noises introduced by the detection and the servo loop are independent in each frame. For a given star, $\sigma_{D}^{2}\left(\mathcal{T}_{F}\right)$ decreases with the frame time $\mathcal{T}_{F}$ while we have seen in the previous section that $\sigma_{L}^{2}\left(\mathcal{T}_{F}\right)$ is proportional to $\mathcal{T}_{F}$. Fig. 12 shows the combination of the detection and loop errors as a function of the frame time for various source magnitudes for the GFT and HFT fringe tracking concepts. We see that for each magnitude we have an optimum frame time that minimizes the tracking error. For a given tracking quality specification (for example $\frac{\lambda}{10}=0.2 \mu \mathrm{m}$ in the plot), we have the optimum frame time and the corresponding limiting tracking coherent magnitude. From Fig. 12 for the UTS at Paranal, and from similar figures for ATs at Paranal and $1.8 \mathrm{~m}$ telescopes at Dome $\mathrm{C}$, we derive the optimum exposure times and limiting magnitudes given in Table 3 .

Note that in this fringe tracking model where we would be limited only by the optical turbulence, for a $\frac{\lambda}{10}=0.2 \mu \mathrm{m}$ tracking precision in the $K$ band, the optimum exposure time is quite large with $U T s$, of the order of $31 \mathrm{~ms}$, almost independently from the fringe tracking design and number of collected photons.

With the GFT and HFT values given in Section 2.7, this yields limiting magnitudes of $\mathrm{K}=13.5$ with the GFT and $\mathrm{K}=15.9$ with the HFT. This optimum exposure time sets a goal for the damping of VLTI vibrations and for the stability of AO that should be compatible with such frame times. As we cannot be sure that such long exposure times will indeed be permitted by VLTI and AO perturbations, we have also considered a maybe more realistic limit at $\mathcal{T}_{F}=10 \mathrm{~ms}$ that yields $\mathrm{K} \sim 13$ with the GFT and $\mathrm{K} \sim 15.3$ with the HFT. We see that the limitation to a shorter frame time as a moderate impact as around the optimum time $\mathcal{T}_{F}$, the variance $\sigma_{D}^{2}\left(\mathcal{T}_{F}\right)$ does not vary much in a relatively large range.

For a given FT, site, and seeing conditions, each $K$ magnitude sets an optimum frame time $\mathcal{T}_{F O}$, which minimizes $\sigma_{F}^{2}\left(\mathcal{T}_{F O}\right)=$ $\sigma_{D}^{2}\left(\mathcal{T}_{F O}\right)+\sigma_{L}^{2}\left(\mathcal{T}_{F O}\right)$. For computational efficiency, we define a function $\mathcal{T}_{F O}=f(K, z)$ that gives the optimum frame time as a function of the magnitude $\mathrm{K}$ and the zenith distance, as illustrated by Fig. 13, and we fit it with a polynomial function. Then we use the proportionality between $\sigma_{F}=a \mathcal{T}_{F} O$ indicated in Fig. 12 .

\subsection{Chromatic variation of the phase delay}

For fringe tracking in one spectral band around wavelength $\lambda_{t}$ for observations in another spectral band around wavelength $\lambda_{o}$ we have to consider the chromatic dispersion of the OPD between the different interferometric beams. An immediate application would be the use on the VLTI of fringe tracking in the $K$ band (GFT) or in a combination of $J, H$, and $K$ bands (HFT) for observations with MATISSE in the $L, M$, and $N$ bands. Another application will be a $J$ band instrument on the $V L T I$ with the same FTs. And a new PFI interferometer would have to track in the near-IR for observations in the $N$ and $Q$ bands.

The inter-band piston differences are due to the chromatic differences in the air refraction index. For interferometers like the VLTI with delay lines in the air, the largest chromatic effect will result from the large difference in air path between the telescopes and the focal laboratory. For all interferometers, including those with delay lines in evacuated pipes, the turbulent atmosphere introduces homogeneity that has a chromatic component. In the near-IR, the available models (Mathar 2007) for the variation of the refractive index $n(\lambda, T, P, H)$ with wavelengths as a consequence of variation of temperature, pressure, and humidity allowed the correct computation and removal of the OPD between the $J, H$, and $K$ bands in the VLTI instrument AMBER (Petrov et al. 2007) introduced by the difference in air path in the Delay Line Tunnels. They also allowed (Vannier et al. 2004) to evaluate the phase delay chromatic noise introduced by the dry air turbulence at $\sim 2 \times 10^{-4} \mathrm{rad}$ and by the wet air turbulence at $\sim 2 \times 10^{-2} \mathrm{rad}$. These noise levels have no impact on the fringe tracking quality, as it was confirmed by successful fringe tracking in the $H$ band with FINITO (Gai et al. 2004) 
Table 3. Seeing parameters, optimum frame time $\mathcal{T}_{\text {opt }}$ (optimum $\mathcal{T}_{F}$ ), limiting magnitude for $\mathcal{T}_{\text {opt }}$ and limiting magnitude for $10 \mathrm{~ms}$ to achieve $0.2 \mu \mathrm{m}$ for different telescope diameters, sites, and fringe tracking performances.

\begin{tabular}{|c|c|c|c|c|c|c|c|}
\hline Tel. D, site, and FT & Seeing conditions & Seeing(arcsecond) & $\mathcal{T}_{0}(\mathrm{~ms})$ & $\mathcal{T}_{\text {opt }}(\mathrm{ms})$ & $K_{\text {lim }}^{\mathcal{T}_{\text {opt }}(\mathrm{ms})}$ & $K_{\text {lim }}^{10 \mathrm{~ms}}$ & Outer scale $L_{0}(\mathrm{~m})$ \\
\hline \multirow{2}{*}{$8 \mathrm{~m}$ Paranal GFT } & Median & 0.7 & 3.2 & 20.4 & 13.22 & 12.92 & 22 \\
\hline & Best (20 per cent) & 0.6 & 4.4 & 29.8 & 13.47 & 12.96 & 16 \\
\hline \multirow{2}{*}{$8 \mathrm{~m}$ Paranal HFT } & Median & 0.7 & 3.2 & 20.2 & 15.62 & 15.31 & 22 \\
\hline & Best (20 per cent) & 0.6 & 4.4 & 29.2 & 15.9 & 15.35 & 16 \\
\hline \multirow{2}{*}{$1.8 \mathrm{~m}$ Paranal GFT } & Median & 0.7 & 3.2 & 10.2 & 9.48 & 9.46 & 22 \\
\hline & Best (20 per cent) & 0.6 & 4.4 & 15 & 9.75 & 9.65 & 16 \\
\hline \multirow{2}{*}{$1.8 \mathrm{~m}$ Paranal HFT } & Median & 0.7 & 3.2 & 10.2 & 11.82 & 11.80 & 22 \\
\hline & Best (20 per cent) & 0.6 & 4.4 & 14.7 & 12.15 & 12 & 16 \\
\hline \multirow{2}{*}{$1.8 \mathrm{~m}$ Dome C HFT } & Median & 0.84 & 7.9 & 35.9 & 14.4 & 13.4 & 7.34 \\
\hline & Best ( 25 per cent) & 0.43 & 12 & 53.8 & 14.8 & 13.4 & 5 \\
\hline
\end{tabular}

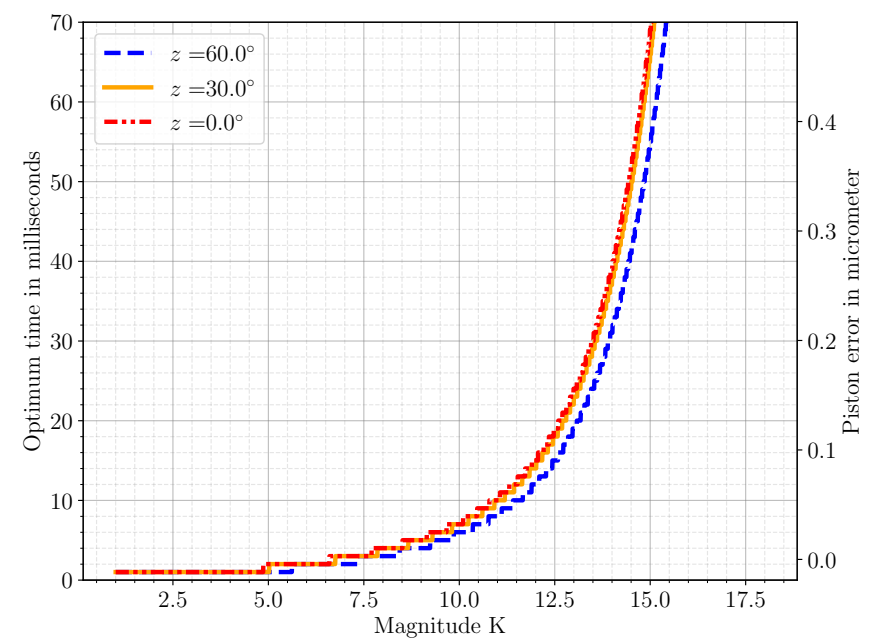

Figure 13. Optimum frame time and piston error as a function of the magnitude $K$ for GFT-UT, for various zenith distances $z=0,30^{\circ}$ and $60^{\circ}$.

for $A M B E R J, H$, and $K$ bands. Therefore, the chromatic OPD effects between the near-IR bands should be considered only for high dynamic observations such as extrasolar planets characterization that are beyond the scope of this paper. A $J+H+K$ FT should therefore only correct for slowly varying chromatic OPD terms to avoid SNR losses due to fringe contrast reduction in broadband channels. Fringe tracking in the $K$ band for observations in the $N$ band has been first implemented on the Keck interferometer (Colavita et al. 2013). The use of the GRAVITY FT in the $K$ band to stabilize the fringes of MATISSE in the $L, M$, and $N$ bands has also been recently commissioned at the VLTI. With the Keck interferometer (Koresko et al. 2006) reported group delay fluctuations of the order of $10 \mu \mathrm{m}$ PTV and $3 \mu \mathrm{m}$ RMS in $N$ while fringes were tracked in $N$. In the same paper, he describes and tests a method to predict that chromatic OPD term from the difference between phase delay and group delay in the $K$ band. That difference is dominated by variations of the water vapor column height that also produce the differences between the $K$ band an $N$ band phase delay difference. This method allowed the prediction and correction of the chromatic phase delay difference below $1 \mu \mathrm{m}$. On the VLTI, while we were accurately tracking in the $K$ band with a residual piston smaller than $0.2 \mu \mathrm{m}$, we observed in $N$ band at $8.5 \mu \mathrm{m}$ fast phase delay fluctuations of about $0.5 \mu \mathrm{m}$ on top of slower group delay fluctuations of about $2 \mu \mathrm{m}$. At $12.5 \mu \mathrm{m}$, these numbers are typically multiplied by 3 . Applying Koresko's method to compute and correct these chromatic effects from the difference observed between the GFT phase and group delays in $K$, we reduce the chromatic OPD fluctuations to $\lambda / 100$ at $8.5 \mu \mathrm{m}, \lambda / 57$ at $10.5 \mu \mathrm{m}$, and $\lambda / 33$ at $12.5 \mu \mathrm{m}$. These are rms values estimated on a sample of eight calibrators observed in a range of fair to good seeing conditions. We do not yet have the database nor the model to evaluate these chromatic residuals in all conditions, so we will use the conservative value of

$\sigma_{H}=0.2 \mu \mathrm{m}$,

for the chromatic OPD error added by tracking in $K$ band for observations at $8.5 \mu \mathrm{m}$.

\section{SKY COVERAGE MAPS}

\subsection{Definition and utility}

To find an off-axis guide star for a specific target for a given tracking specification, for example $\sigma_{T}\left(\lambda_{o}\right)<\lambda_{o} / N_{\text {spec }}$, we have to consider all possible guide stars in a radius $\theta_{p}\left(\lambda_{o}\right)$ around the target and compute the tracking residual for each candidate using equation (7) combined with equations (37, 43, 51 and 59). The target can be tracked off-axis if we find at least one guide star such as:

$$
\begin{aligned}
& \sigma_{D}^{2}\left(\lambda_{t}, J, H, K, \mathcal{T}_{F}\right)+\sigma_{L}^{2}\left(\lambda_{t}, \mathcal{T}_{F}\right)<\left(\frac{\lambda_{t}}{N_{\text {tra }}}\right)^{2} \text { and } \\
& \sigma_{D}^{2}\left(\lambda_{t}, J, H, K, \mathcal{T}_{F}\right)+\sigma_{L}^{2}\left(\lambda_{t}, \mathcal{T}_{F}\right)+\sigma_{H}^{2}\left(\lambda_{t}, \lambda_{o}\right) \ldots, \\
& +\sigma_{A}^{2}\left(\lambda_{o}\right)<\left(\frac{\lambda_{o}}{N_{\text {spec }}}\right)^{2}
\end{aligned}
$$

where $\mathrm{J}, \mathrm{H}$ and $\mathrm{K}$ are the guide star magnitudes in the corresponding spectral bands and $\mathcal{T}_{F}$ is the frame time that can be the optimum frame time set by the guide star magnitude, as explained in Section 2.11 or a fixed frame time set by the FT limits. This approach is used in Section 4 where we discuss two examples of target lists for large AGN programs on the VLTI.

To be able to evaluate the sky coverage for any kind of observation, with target lists yet unknown, it is practical to compute sky coverage maps that give the probability to find at least one usable guide star for any target in any region of the sky. These maps can be used to guide the preselection of science target lists and also to specify the required performances of the FT and the wide field selector, which has an impact on their design or development plan.

\subsection{Methodology}

The first step is to define a database of possible guide stars. We have used the $2^{\text {nd }}$ data release of Gaia survey (Gaia Collaboration 2018) ${ }^{1}$ that covers astrometry, photometry, radial velocities, and astrophysical parameters of 1.692.919.135 extrasolar objects. Although, this survey does not give directly the $\mathrm{K}$ magnitudes, we expected it to be more

\footnotetext{
1 https://gea.esac.esa.int/archive/documentation/GDR2/
} 
complete for faint stars than the 2MASS catalogue which was initially specified to reach only magnitude $\mathrm{K} \simeq 14.3$ (Skrutskie et al. 2006), while we planned to explore precisely the domain between $\mathrm{K}=14$ and $K=16.5$. Histograms of the number of stars as a function of the $K$ magnitude showed that both the Gaia DR2 and 2MASS list seemed complete up to $\mathrm{K}=17$ for Gaia and $\mathrm{K} \simeq 16.5$ for $2 \mathrm{MASS}$. Although the magnitudes of many of the targets fainter than $\mathrm{K} \simeq 15$ are flagged as very uncertain in the 2 MASS catalogue, it should give quite similar sky coverage maps. Finally, the justification to stay with the Gaia data base is its astrometric quality. With the maximum VLTI baseline of $200 \mathrm{~m}$, an error of 1 arcsecond on the position of the guide star results in an error of $1 \mathrm{~mm}$ on the zero OPD on the science fringes. This is much larger than the typical low resolution coherence lengths that range from $60 \mu \mathrm{m}$ to $300 \mu \mathrm{m}$ from $K$ to $N$. Scanning to find the science fringes is problematic on faint targets. The fringe tracking specification of $10 \mu \mathrm{m}$, to be sure to be within the coherence lenght, corresponds to an astrometric precision of 10 milliarcsecond, which is achieved by Gaia even on the faintest targets (Lindegren et al. 2018) but well below the nearly 1 arcsecond 2MASS astrometric precision. Moreover, the 1 milliarcsecond Gaia astrometric precision would allow a science instrument astrometry of 1 milliarcsecond that would allow the registration of the images obtained in the different science spectral bands with the resolution of the $J$ band. Gaia observed 1.381.964.755 sources in the blue colour index $\left(G_{B P}\right.$-band: $\left.330-680 \mathrm{~nm}\right)$ and 1.383 .551 .713 objects in the red colour indexes $\left(G_{R P}\right.$ - band: $\left.630-1050 \mathrm{~nm}\right)$. To switch from Gaia bands $\left(G_{R P}\right.$ and $\left.G_{B P}\right)$ to $K$, we used the 2MASS quadratic photometry relationship from Evans et al. (2018), as follows:

$$
\begin{aligned}
\mathrm{G}-\mathrm{K}= & -0.1885+2.092 \cdot\left(\mathrm{G}_{\mathrm{BP}}-\mathrm{G}_{\mathrm{RP}}\right) \\
& -0.1345 \cdot\left(\mathrm{G}_{\mathrm{BP}}-\mathrm{G}_{\mathrm{RP}}\right)^{2} .
\end{aligned}
$$

This formula is valid only if the difference between the blue and red colour index is between 0.25 and $5.5\left(0.25<\mathrm{G}_{\mathrm{BP}}-\mathrm{G}_{\mathrm{RP}}<5.5\right)$. This validity condition also sets a high probability to be observing a source without too strong IR excesses that would compromise the K magnitude estimate. We selected sources that have a magnitude $K \leq 17$, which is one magnitude above the best limit of our FTs.

This leaves us with a database of 814.728.004 targets as an input for our sky coverage maps (including 498.346.813 targets brighter than $\mathrm{K} \leq 16$ ). Note that we do not have a guarantee that all these sources are good calibrators for interferometric observations as we cannot be sure that they are single unresolved stars. However, an overwhelming majority should be good enough for fringe tracking. In the range of magnitudes $\mathrm{K}$ from 10 to 16 that are relevant to our study, it is extremely unlikely that sources without strong IR excess are resolved enough to lower the contrast of fringes on $100 \mathrm{~m}$ baselines. Even a supergiant like Betelgeuse would have an angular size smaller than 0.1 milliarcsecond (mas) if it is far enough to have an observed magnitude $\mathrm{K}>10$. Stars brighter than $\mathrm{K} \sim 5$ could start to be resolved, but their number is negligible (and many of them are already out of the Gaia observation list to avoid saturating its detectors). A small number of binary stars would not be suitable for fringe tracking, but they should be eliminated only if the separation is smaller than the single aperture Airy radius in $K(50$ mas for $\mathrm{D}=8 \mathrm{~m})$ and the magnitude difference is smaller than 1 . This would eliminate a very small fraction of the candidates, without significantly changing the sky coverage percentages.

Our database contains only the $K$ band magnitude. As our HFT options uses also some flux in $H$ and in $J$, we have used fixed magnitude differences that are the mean values for our reduced database:

$H-K=0.1$ and $J-K=0.5$.

In future versions of our sky coverage map calculator, we will implement a database containing magnitude estimates in the other bands. They will be necessary to validate specific guide stars but should have a very marginal impact on sky coverage maps.

To estimate the probability to find a guide star in any position of the sky, independently from any specific target list, we divide the sky in sampling pixels of $(10 \text { arcsecond })^{2}$ of fixed surface whatever the declination (i.e. the sampling step in right ascension is $\left(\frac{10 \text { arcsecond }}{\cos (\delta)}\right)^{2}$. This sampling step is set by the limits of the RAM available to compute the maps. As the stellar density does not evolve on much smaller angular scales except in very special areas of the sky, we estimate that it is more than sufficient for the general sky coverage maps that we are considering here, and indeed a sampling of ( 30 arcsecond $)^{2}$ gives very similar results. Then we examine each one of the sources in our database. From their $K$ magnitude, we define an optimum frame time $\mathcal{T}_{F O}$ as in Section 2.11. If there is a set frame time $\mathcal{T}_{F S}$, we use the optimum frame time only if it is smaller than the set time:

$\mathcal{T}_{F}=\min \left(\mathcal{T}_{F O}, \mathcal{T}_{F S}\right)$

Then we have $\sigma_{F}^{2}\left(\lambda_{t}, J, H, K, \mathcal{T}_{F}\right)=\sigma_{D}^{2}\left(\lambda_{t}, J, H, K, \mathcal{T}_{F}\right)+$ $\sigma_{L}^{2}\left(\lambda_{t}, \mathcal{T}_{F}\right)$. If $\sigma_{F}^{2}\left(\lambda_{t}, J, H, K, \mathcal{T}_{F}\right)>\left(\frac{\lambda_{t}}{N_{\text {tra }}}\right)^{2}$, the tracking noise is too large and this star does not contribute to the sky coverage. If it could contribute, we examine all the pixels in its vicinity within a radius $\theta_{P}\left(\lambda_{o}\right)$. From the angular distance $\theta$ between the source and the centre of the sky map pixel, we compute $\sigma_{A}\left(\lambda_{o}\right)$. Then, if $\lambda_{t} \neq \lambda_{o}$, we add the fixed $\sigma_{A}\left(\lambda_{o}\right)^{2}$ defined in Section 2.12. If the resulting total variance from equation (7) $\sigma_{T}^{2}<\left(\frac{\lambda_{o}}{N_{\text {spec }}}\right)^{2}$ we set the examined pixel to 1 , which means that there is at least one guide star for a target in that pixel. Finally, the sky coverage map is given in larger pixels of $\left(0.5^{\circ 2}\right.$ by dividing the number of $(10 \text { arcsecond })^{2}$ pixels set to 1 by the total number of $(10 \text { arcsecond })^{2}$ pixels in that $\left(0.5^{\circ 2}\right.$ pixel, which gives the probability for a target randomly placed in that sky map pixel to find at least one usable off-axis guide star.

We have investigated the following sites and telescopes:

(i) $8 \mathrm{~m}$ telescopes $(U T S)$ at Paranal Observatory.

(a) GFT and HFT in a GRAVITY + context, for observations in the $K$ band.

(b) GFT and HFT in a GRAVITY + context, for observations in the $N$ band with MATISSE.

(ii) $1.8 \mathrm{~m}$ telescopes $(A T s)$ at Paranal Observatory.

(a) GFT and HFT for observations in the $K$ band.

(b) GFT and HFT for observations with MATISSE in the $N$ band.

(iii) PFI with $1.8 \mathrm{~m}$ telescopes at a site like Paranal.

(a) HFT for observations in the $N$ band.

(iv) PFI with $1.8 \mathrm{~m}$ telescopes at Dome C in Antarctica, assuming that the telescopes are above the $30 \mathrm{~m}$ ground layer.

(a) HFT for observations in the $K$ and $N$ bands.

We have considered median seeing conditions as well as the seeing conditions that occur in the best 20 per cent of the time. The corresponding parameters of these sites are given in Table 3.

\subsection{VLTI with UTs}

Fig. 14(a) shows the sky coverage that should be obtained with the GFT for observations in the $K$ band, with the seeing expected in the best 20 per cent conditions. The sky coverage remains limited far from the Galactic Plane as it drops below 10 per cent at $\pm \sim 30^{\circ}$ of the Galactic Plane. However, this is sufficient for a program based on a large number 


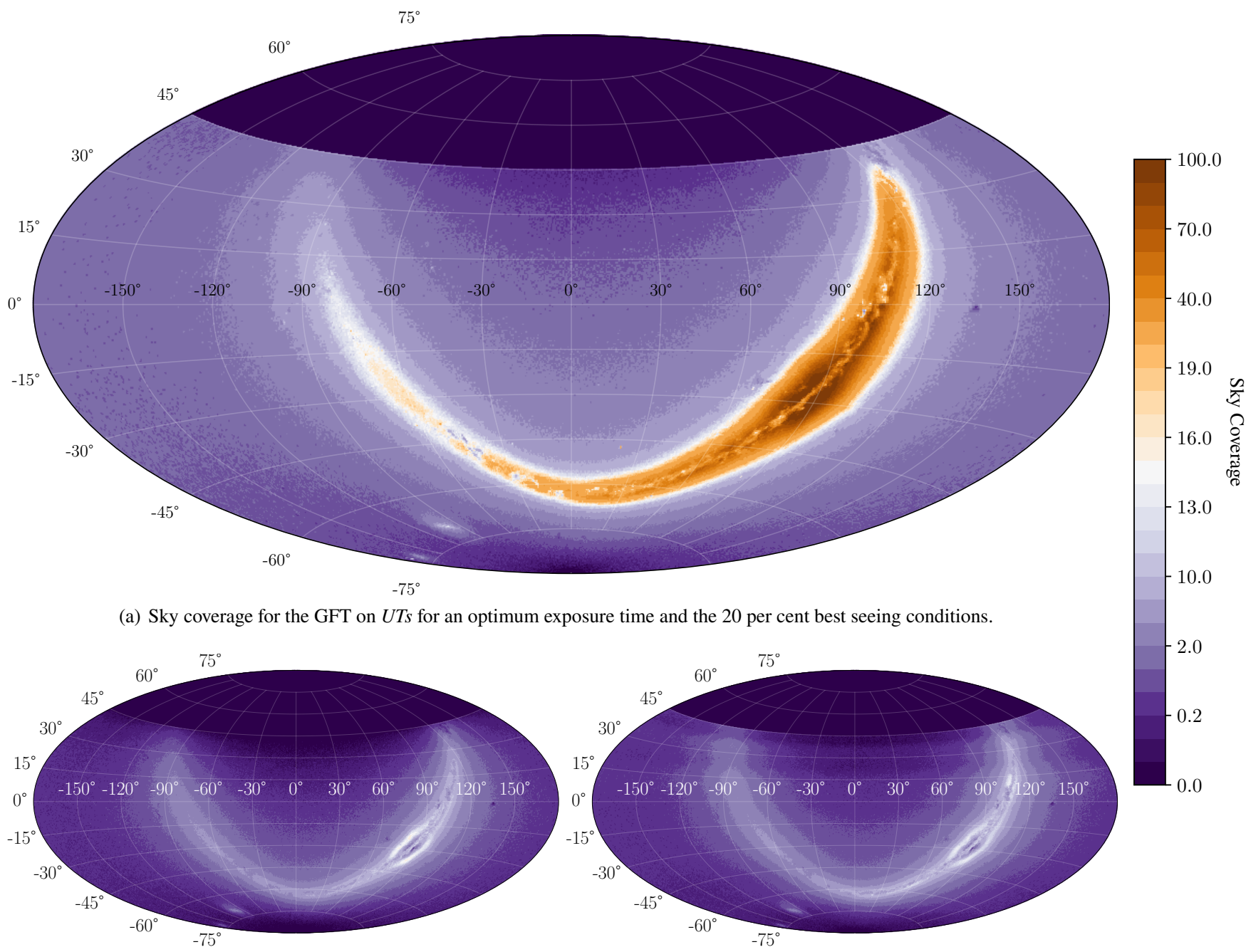

(b) Sky coverage difference between optimum and $10 \mathrm{~ms}$ exposure time (best 20 per cent seeing).

(c) Sky coverage difference between 20 per cent best and median seeing (optimum exposure time).

Figure 14. Sky Coverage expected with the GRAVITY+ upgrade and the current GRAVITY FT tracker. The top image shows the sky coverage with an optimum exposure time (that can reach $30 \mathrm{~ms}$ ) in the best 20 per cent seeing conditions. The sky coverage remains limited far from the Galactic Plane as it drops below 10 per cent at $\pm 30^{\circ}$ of the Galactic Plane in the best case. However, this is sufficient to undertake a large AGN program as we will see in Section 4.5. The two lower images show the impact of a frame time limited to $10 \mathrm{~ms}$ (left) and a seeing in median conditions (right). The impact is significant without radically changing the sky coverage.

of sources evenly spread over the sky, as we will see it for a large AGN program in Section 4.5 below.

Fig. 14(b) shows the difference between observations with the optimum frame time, which can reach $30 \mathrm{~ms}$ (see Fig. 12), and observations with a maximum frame time set at $10 \mathrm{~ms}$. The gain offered by the optimum frame time is significant as it extends the 10 per cent limit by about $10^{\circ}$ away from the Galactic Plane. Fig. 14(c) shows the difference between the sky coverage in the best 20 per cent seeing conditions and in median seeing conditions. The seeing conditions do not change the general shape of the sky coverage, but should be considered carefully for targets with a guide star at the limit of the performances

Fig. 15 shows the sky coverage with the new generation FT evaluated in Section 2.7. The sky coverage improvement offered by the gain of two magnitudes on the tracking limit is quite substantial, with a sky coverage larger than 10 per cent of the sky observable from Paranal.

Fig. 16 shows the sky coverage with the GFT and the HFT for observations in the $N$ band with MATISSE, in the same conditions, with a separation between the target and the guide star limited to 1 arcminute.
That sky coverage is only slightly better than this for $K$ band observations. However, the isopistonic angle for observations in the $N$ band with UTS is 174 arcsecond at $8.5 \mathrm{~ms}$ in median seeing conditions and can reach 230 arcsecond in the best 20 per cent conditions. Extending the off-axis range to these values yields a spectacular increase in the sky coverage as shown by Figs 16(c) and 16(d) where the separation is still limited to 2 arcminute for hardware realism.

\subsection{VLTI with ATs}

We have also investigated the sky coverage that can be expected with the $A T s$. It is reduced first because the fringe tracking limiting magnitude is very substantially reduced, by 3.2 magnitudes if nothing changes except the telescope diameter. The smaller apertures also result in faster turbulence and hence increased loop error $\sigma_{L}$ and in a much smaller isopistonic angle and increased anisopistonic error $\sigma_{A}$. As a consequence, the sky coverage with the GFT on the ATs for observations in the $K$ band is less than a marginal, with less than 1 per cent even in the highest guide star density. With the HFT we have values that can 


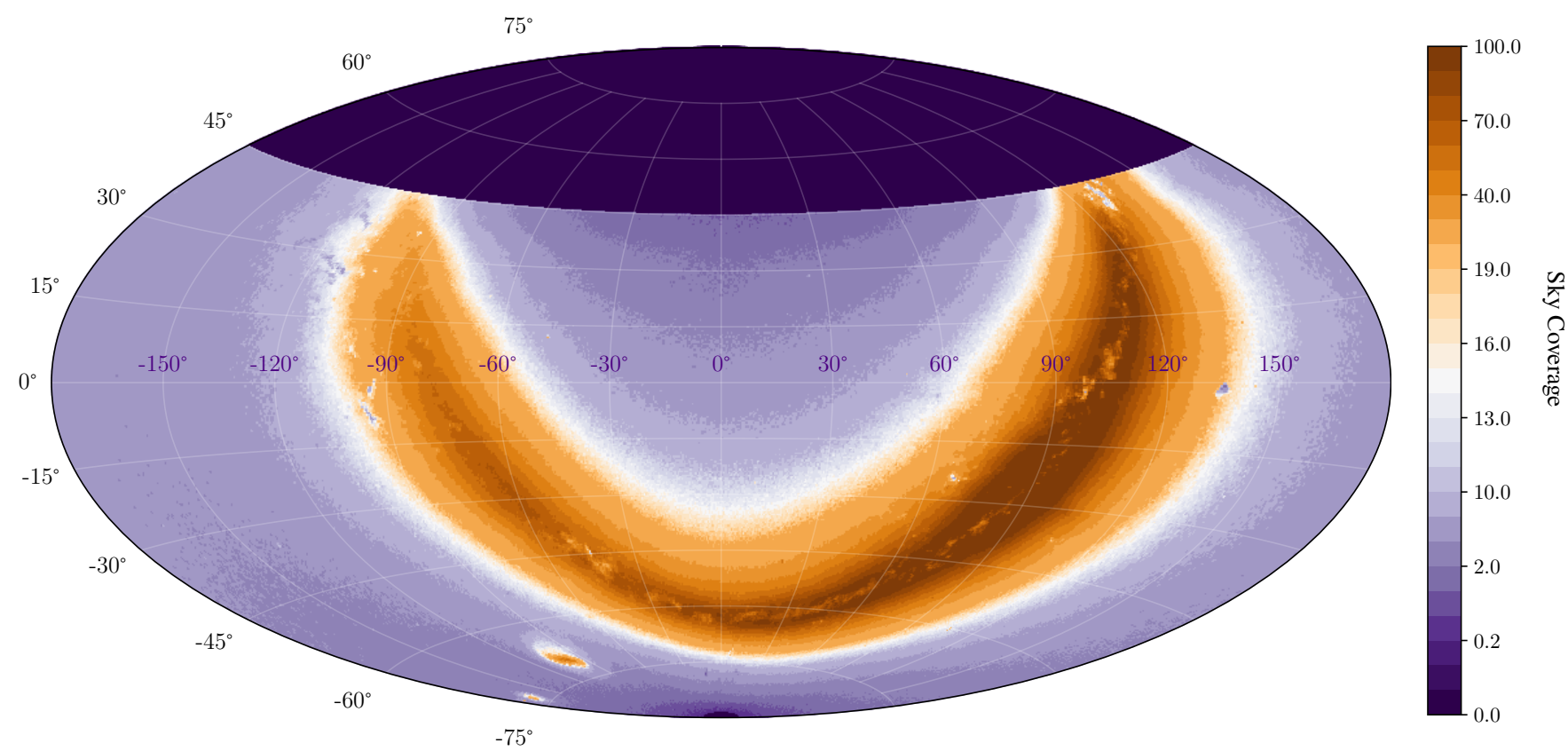

Figure 15. Sky coverage in the best 20 per cent seeing conditions and optimum exposure time with the new generation HFT evaluated in Section 2.7. This figure illustrates the strong gain in sky coverage allowed by a gain of two magnitudes on the fringe tracking sensitivity limits.

exceed 20 per cent near the Galactic Centre and 10 per cent almost everywhere very close to the Galactic Plane, as shown in Fig. 17. This might be worth it for classes of programs with targets near the Galactic Plane. For observations in the $N$ band, we have a strong increase in sky coverage, as shown by Fig. 18. With the GFT we will have a limited but sometimes usable sky coverage near the Galactic Plane and we will see on the AGN example below that this already provides some high-interest targets to boost the image reconstruction capability of MATISSE with the ATs in the $N$ band. This will include YSOs that are too resolved for 'on-axis'.

\subsection{PFI with $1.8 \mathrm{~m}$ telescopes in a Paranal like site}

The sky coverage with an HFT for ATs at Paranal shown in Fig. 17 for the $K$ band, and Fig. 19 for the $N$ band, should be extremely similar to this with a new generation interferometer, like the PFI, with any number of telescopes in a site with the same seeing conditions as Paranal. Indeed with an HFT, the fringe tracking performances are independent of the number of apertures, at least for unresolved targets such as the off-axis guide stars. This sky coverage for $N$ band observations is more than sufficient for the main science goal of PFI which is the detailed imaging of protoplanetary discs. Showing that this can be achieved with $1.8 \mathrm{~m}$ class telescopes has indeed a remarkable impact on the cost and hence the feasibility of such an array. The preliminary studies of the PFI also showed that its overall transmission could be substantially improved with regard to the VLTI, because PFI would be an optimized interferometer unlike the VLTI that is an interferometric mode added to a general-purpose multitelescope observatory.

\section{EXAMPLE OF APPLICATION: AN AGN LARGE PROGRAM}

To give a more concrete example of the potential of off-axis fringe tracking we have considered two target lists for a science program on AGNs. Such a science program would include several components that are briefly described in the following with their requirements.

\subsection{Phase differential interferometry of Broad Line Regions}

The spectro-astrometric study of AGN BLRs by phase differential interferometry (PDI) in emission lines, proposed by (Petrov et al. 2001) and successfully achieved by GRAVITY (Gravity Collaboration 2020c), can constrain the kinematics, size, and geometry of a spatially unresolved BLR using the displacement of the AGN photocentre with wavelengths through an emission line (that is proportional to the differential phase on unresolved sources). It can be used to evaluate the mass of the SMBH in the centre of the AGN (Rakshit et al. 2015). Combined with Reverberation Mapping that gives an equivalent linear size of the BLR, the angular size given by differential interferometry yields a direct distance measurement of the BLR (Wang et al. 2020b). A study on a large number of BLRs up to $z=2$ is one of the main motivations for the GRAVITY + program. The key condition to realize it is the ability to observe a large number of Seyfert 1 AGNs and QSOs up to magnitude $\mathrm{K} \leqslant 17$.

GRAVITY+ intends to use the GRAVITY science instrument and is focused on off-axis fringe tracking in the $K$ band with the GFT and science observation at medium $(R=1500)$ or high $(R=4500)$ spectral resolution in the $K$ band. Observing strong emission lines in the $K$ band requires targets with high enough redshifts to have Paschen $\alpha, \beta, \gamma$, and $\delta$, Helium I or even $H \alpha$ lines shifted in the $K$ band. High redshift means distant and that implies that bright enough targets are large and hence difficult to perform Reverberation Mapping on. A complement to the $G R A V I T Y+K$ band program would be the $J$ band instrument proposed by Petrov et al. (2019) that allows observing the same lines with a much smaller redshift and hence on closer and smaller targets more likely to allow RM measurements. The condition for these observations are:

(i) To have an off-axis target for fringe tracking in the near-IR.

(ii) A fringe tracking precision $\sigma_{T} \simeq 0.2 \mu \mathrm{m}$ for observations in the $K$ band.

(iii) A fringe tracking precision $\sigma_{T} \simeq 0.1 \mu \mathrm{m}$ for observations in the $J$ band.

In addition to these fringe tracking requirements, we need the PDI SNR to be sufficient. On unresolved targets (i.e. with size $\Omega$ much 


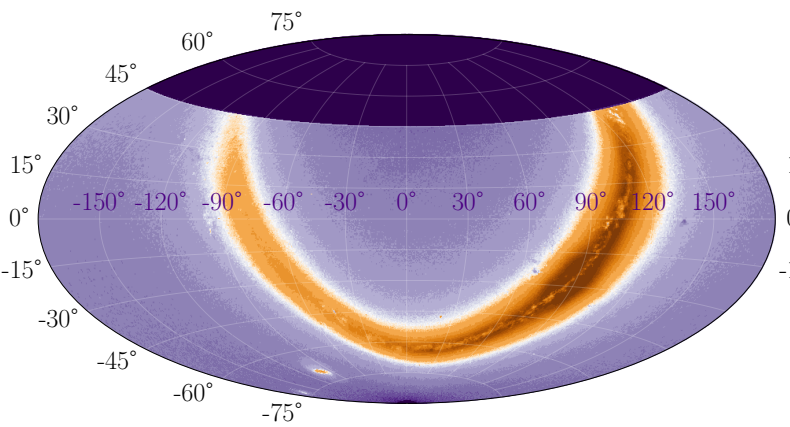

(a) GFT for MATISSE - max separation = 1 arcminute.

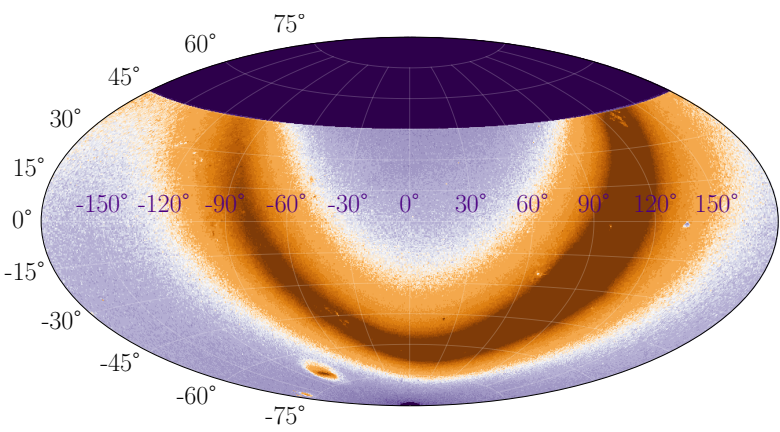

(c) GFT for MATISSE - max separation = 2 arcminutes.

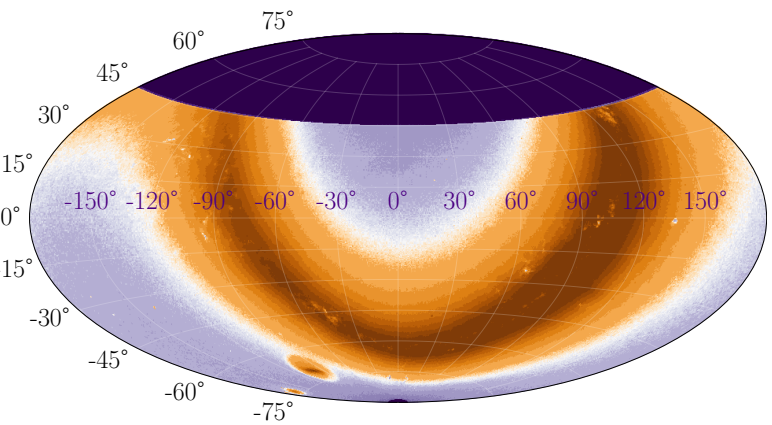

(b) HFT for MATISSE - max separation = 1 arcminute.

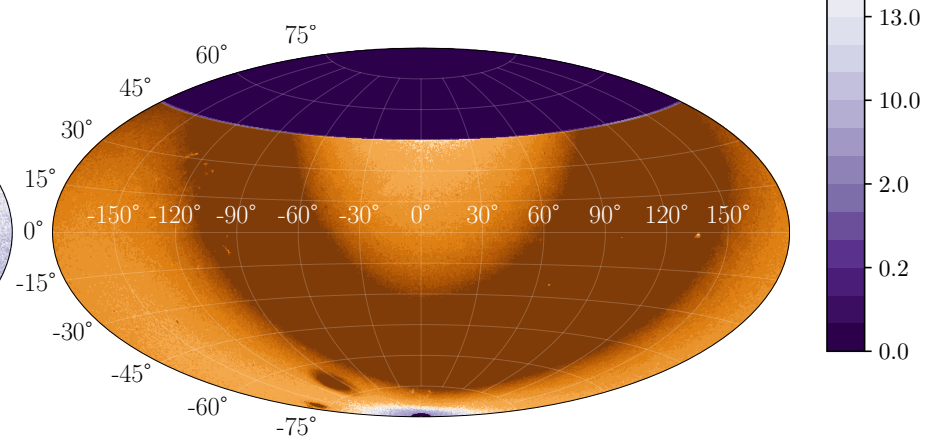

(d) HFT for MATISSE - max separation $=2$ arcminutes.

Figure 16. Sky coverage for tracking in the $K$ band with the GFT (top-left) and the HFT (top-right) for observations in the $N$ band with MATISSE. Optimum exposure time and best 20 per cent seeing. Top row: target separation limited to 1 arcminute. Bottom row: target separation limited to 2 arcminutes that remain smaller than the isopistonic angle with $U T s$, which is strongly relaxed for $N$ band observations with $U T S$.

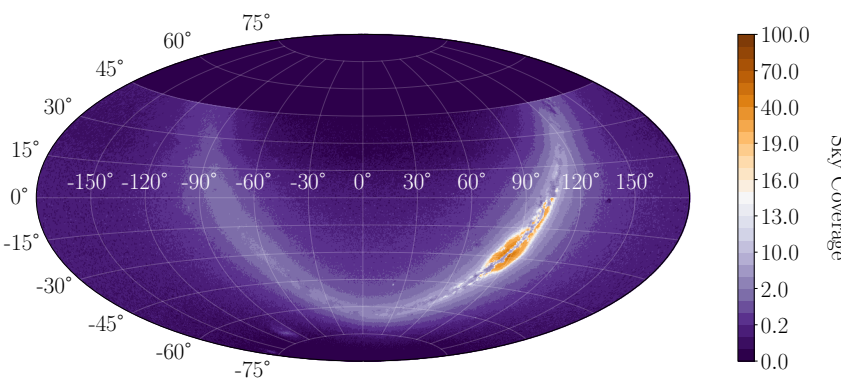

Figure 17. Best Sky coverage with an HFT on ATs (or with a $1.8 \mathrm{~m}$ class telescopes at a PFI interferometer in a site like Paranal) for observations in the $K$ band. The sky coverage exceeds 10 per cent only within $\pm 10^{\circ}$ around the Galactic Plane. With the GFT, the sky coverage in the same conditions is negligible.

smaller than the resolution limit $\lambda / B$ ) the differential phase signal is given by (Petrov et al. 1986)

$\phi(\lambda)=2 \pi \epsilon(\lambda)(B / \lambda)$,

where $\epsilon(\lambda)$ is the photocentre variation of the source at the wavelength $\lambda$. In an emission line, the photocentre variation with regard to the nearby continuum (where the photocentre is set to zero) is $\epsilon(\lambda)=k(\lambda) \Omega$ where $k(\lambda)$ depends from the geometry of the target and the strength of the line. For emission lines much stronger than the continuum, $k_{\text {max }} \simeq 0.5$. The differential phase noise is $\sigma_{\phi}(\lambda)=\frac{1}{S N R_{C}(\lambda) \sqrt{2}}$ (Petrov et al. 2020) and the Phase Differential Interferometry $S N R_{P D I}$ for unresolved targets can thus be written as:

$S N R_{P D I}(\lambda) \simeq \pi \sqrt{2} \frac{\Omega_{B L R}}{\lambda / B} \operatorname{SNR}_{C}(\lambda)$.

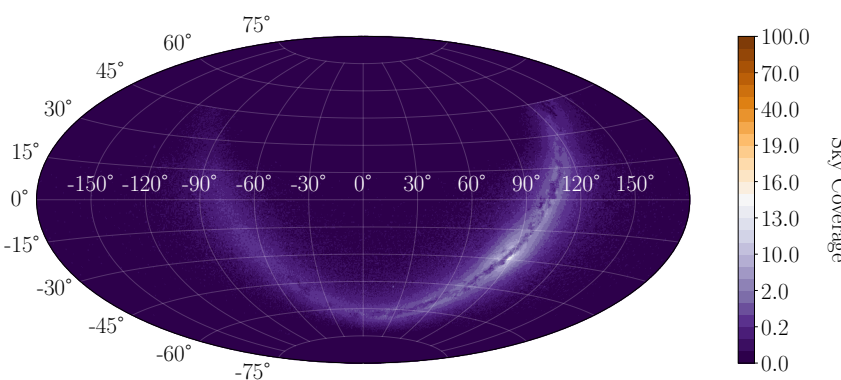

Figure 18. Best sky coverage with a GFT on ATs for observations with MATISSE. That sky coverage would be achievable with the GFT 'as it is' with a periscopic system allowing feeding in GRAVITY and MATISSE two sources with separations up to 1 arcminute. The sky coverage might seem modest but it immediately opens very interesting science programs as it would allow imaging and high spectral resolution observations for already a few nearby AGNs, as shown in Fig. 25.

In the following, we should consider that a condition for a good spectro-astrometric information is given by:

$\min \left[\frac{\Omega_{B L R}}{\lambda / B}, 1\right] S N R_{C}(\lambda)>10$,

The term min $\left[\frac{\Omega_{B L R}}{\lambda / B}, 1\right]$ expresses the fact that the differential phases stop increasing with the size $\Omega$ on resolved objects. The criteria given here correspond to an error on the BLR angular size of $\simeq 1$ per cent in the case of thin flat Keplerian BLR observed in a strong emission line (Rakshit et al. 2015). 


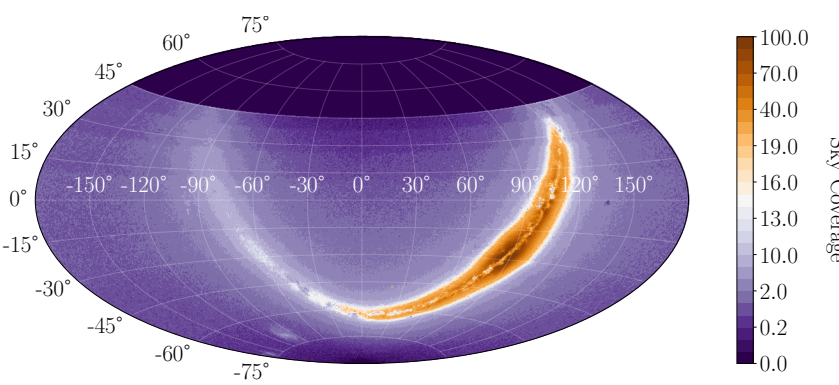

Figure 19. Best sky coverage with an HFT for observations in the $N$ band with ATs in Paranal or with PFI with $1.8 \mathrm{~m}$ apertures in a Paranal like site. The overall transmission was assumed to be the same as with the VLTI. In this figure as well as in Figs 17 and 18, we assumed an optimum exposure time and the 20 per cent best seeing conditions.

\subsection{Amplitude differential interferometry of BLRs}

The interpretation of spectro-interferometric measures alone as well as combined with Reverberation Mapping alone is strongly model dependent and different BLR geometries can give very different SMBH masses and distance estimates from the same measurements as shown by Rakshit et al. (2015). The same paper shows that amplitude differential interferometry (ADI), i.e. the variations of the amplitude of the coherent flux, or of the visibility modulus through the line very strongly constrains and improves the mass and distance estimates. On unresolved targets the differential visibility drop $1-V(\lambda)$ is proportional to $\left(\frac{\Omega}{\lambda / B}\right)^{2}$ and the $S N R_{A D 1}$ can be estimated by:

$\operatorname{SNR}_{A D I}(\lambda) \simeq \min \left[\left(\frac{\Omega_{B L R}}{\lambda / B}\right)^{2}, 1\right] \operatorname{SNR}_{C(\lambda)}>10$,

The condition $S N R_{A D I}(\lambda) \simeq 10$ corresponds for example to a precision of $\simeq 6^{\circ}$ on the inclination of a thin flat BLR.

\subsection{Imaging of broad line regions}

To definitively validate mass and distance estimates by PDI, even completed by ADI, we need to reconstruct images of BLRs on a sample of sources to solve all possible geometric ambiguities. The largest BLRs are expected to have angular sizes of the order of 1 milliarcsecond or smaller and this is confirmed by the first successful measurements by GRAVITY. Imaging these structures would require baselines of $300 \mathrm{~m}$ in the visible, $600 \mathrm{~m}$ in the $J$ band, and more than $1 \mathrm{~km}$ in the $K$ band with tracking quality of respectively $0.06 \mu \mathrm{m}, 0.01 \mu \mathrm{m}$, and $0.02 \mu \mathrm{m}$. This is out of reach for any existing optical interferometer. The VLTI's current and final maximum baselines are 135 and $200 \mathrm{~m}$ and the CHARA interferometer, which would have a sufficient resolution in the visible with its baselines up to $330 \mathrm{~m}$, cannot be sensitive enough with its $1 \mathrm{~m}$ apertures. In Section 5 below we will discuss with some more details the sensitivity conditions for an optical interferometer to make images of BLRs.

In optical interferometry, the key condition to make images is to obtain closure phase measurements. The closure phase decreases as the third power of the ratio $\frac{\Omega}{\lambda / B}$ and the condition to have a closure phase usable for image reconstruction becomes a condition on the closure phase $S N R_{C P}$ :

$\operatorname{SNR}_{C P}(\lambda) \simeq \min \left[\left(\frac{\Omega_{B L R}}{\lambda / B}\right)^{3}, 1\right] \operatorname{SNR}_{C(\lambda)}>10$.

The possibility to retrieve images from unresolved targets is very limited.
4.4 Constraining the structure of the dust torus with MATISSE

A key component of the unified model of AGNs (Antonucci 1993) is the dust torus that obscures the central emission source in type 2 AGNs, where it is seen 'edge-on', and does not in type 1 AGNs, where it is sufficiently 'face-on'. Optical Interferometry in the mid-IR with MIDI (Burtscher et al. 2013) complicated the long held image of a simple equatorial 'torus', which is now thought to be clumpy and with polar components probably blown away by radiation pressure (the dusty wind) as well as other associated inflows and outflows. The complexity of that structure is confirmed by the first observations of AGN dust tori by GRAVITY (Gravity Collaboration 2017a) and MATISSE. Understanding the structure of the dust in a large sample of AGNs with a large range of luminosities would help constrain and complement the BLR studies described in the previous section by explaining how the accretion disc around the central SMBH exchanges material with the galaxy. IR Reverberation Mapping, which gives linear dust torus sizes, combined with the angular measures of optical interferometry yields direct distance measurements as demonstrated by Hönig et al. (2014). But like for BLRs, the precision of these distance measurements strongly depends on the geometry of the torus. The two topics are strongly correlated, as the geometry of the gas in the BLR might be a prolongation of this of the surrounding dust.

One of the primary science goals of MATISSE (Lopez et al. 2014) is the study of that innermost dust 'torus' in AGNs. In spite of the complexity revealed by optical interferometry, the inner part of the dust structure is likely to be dominated by an inclined (and more or less skewed) ring, complemented by a dusty biconical outflow, or 'wind ', with a strength related to the central source luminosity and hence SMBH mass. In the $K$ and $L$ band, we would be dominated by the bright inner rim near or shortly behind the dust sublimation radius; at longer wavelengths we see colder dust. MATISSE, alone or assisted by the GFT, will be able to observe a few dozen AGN tori and will obtain images of 2 or 3 nearby AGNs. However, that number of targets, as well as the range of available physical conditions in the AGN, is very strongly limited by the current sensitivity limits of the VLTI AO and GFT. So the MATISSE scientific potential will be very strongly enhanced by the GRAVITY+ upgrade.

Similarly to the BLRs, the dust torus observations of MATISSE can be classed in the three categories 'imaging', 'ADI', and 'PDI' according to the ratio size over resolution $\frac{\Omega}{\lambda / B}$ and equations $(65,66$, and 67$)$ apply by changing the wavelength domain and replacing the size of the BLR $\Omega_{B L R}$ by this of the torus $\Omega_{T}(\lambda)$. A key difference is that the torus is much larger than the BLR and imaging, i.e. $\Omega_{T}(\lambda)>\frac{\lambda}{B}$ applies on much large number of targets. In the $K$ band, we will be dominated by the inner rim of the dust. Suganuma et al. (2006) finds that the infrared reverberation mapping size is very close to the dust sublimation radius which is proportional to the square root of the bolometric luminosity. Kishimoto et al. (2011) find an interferometric size in the $K$ band just slightly larger, with an excess that seems loosely correlated to the luminosity. The apparent size of the torus changes with the wavelength of observation and can reach a $\lambda^{2}$ dependence for optically thin dust directly illuminated by the central source. The overview of the observation of 23 AGNs by MIDI by Burtscher et al. (2013) reveals a $\frac{\Omega_{T}\left(\lambda_{N}\right)}{\Omega_{T}\left(\lambda_{K}\right)}$ size ratio that ranges from $\frac{\lambda_{N}}{\lambda_{K}}$ to $\left(\frac{\lambda_{N}}{\lambda_{K}}\right)^{2}$ with a median value of $\frac{\Omega_{T}\left(\lambda_{N}\right)}{\Omega_{T}\left(\lambda_{K}\right)}=9$. We will use these $\Omega_{T}\left(\lambda_{K}\right) \propto L^{1 / 2}$ and $\frac{\Omega_{T}\left(\lambda_{N}\right)}{\Omega_{T}\left(\lambda_{K}\right)}=9$ relations to check what kind of observations MATISSE can obtain on some of the AGNs that are considered in the next sections. 


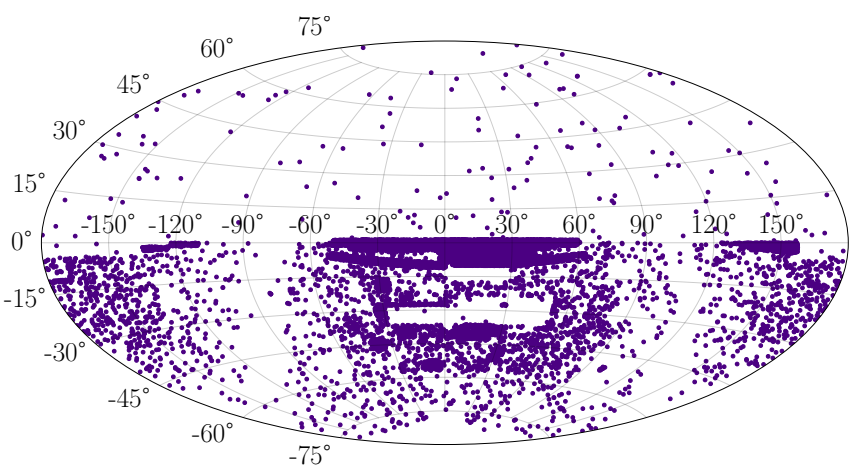

Figure 20. The list of 15799 QSOs used to evaluate the potential of the overall Quasar program. Note the very strong survey biases

\subsection{Large list of Quasars}

We have considered two lists of AGNs to investigate various aspects of the potential of GRAVITY and MATISSE observations in the GRAVITY+ context with off-axis tracking either by the GFT or by the HFT.

The first list contains 15799 Quasars observable with the VLTI. We utilized the PYTHON packages ASTROQUERY (Ginsburg et al. 2019), ASTROPY (Astropy Collaboration 2018), and NUMPY (Harris et al. 2020). We queried Simbad astronomical database (Wenger et al. 2000) for every object classed as a QSO. We selected the QSO classification because this reduces the relative contamination from the host galaxy, minimizing the difference between the catalogue $K$ magnitude and this of the actual central source that is seen by the VLTI. Our list is then a subset of the AGNs observable from the VLTI but a fraction of targets in this list will be eventually fainter than $K=17$. We crossmatched these QSO coordinates with the VISTA Hemisphere Survey (VHS; McMahon et al. 2013). VHS has a Ks limiting magnitude of 18.1 making it appropriate for this study that we restricted it to $K \leqslant 17$. From VHS, we found the $K s$ band magnitude using an aperture of 1 arcsecond and removing any objects with a Ks magnitude greater than 17. The VHS is almost strictly limited to targets with negative declination and misses all targets in the Northern hemisphere that would be observable from Paranal. It also seems to contain some other very strong survey biases, as shown by Fig. 20, but it provides a large number of potential candidates distributed all over the southern hemisphere and we considered it as a usable input to test the number of potential candidates for various types of observations. We have to note that there is a relative deficit of targets near the Galactic Plane and a massive over-representation of targets near declination zero.

The targets for which we find a guide star are labelled as a function of the quality of tracking offered by the best guide star that minimizes the error budget of equations ( 6 and 7). Tracking qualities of $0.2 \mu \mathrm{m}, 0.1 \mu \mathrm{m}$ and $0.06 \mu \mathrm{m}$ allow observations in the $K, J$ and $R$ bands respectively, while tracking qualities of $0.85 \mu \mathrm{m}, 0.42 \mu \mathrm{m}$ and $0.28 \mu \mathrm{m}$ are good for observations in $N, M$ and $L$ respectively.

Figs 21(a) and 21(b) show the targets that would have guide stars with the GFT and the HFT on UTs in the best 20 per cent seeing conditions. The GFT would allow observations in the $K$ band of 1380 of the initial 15799 QSOs, i.e. 8.3 per cent. This is more than enough to support a large program for the observation of BLRs in $K$. These targets are selected strictly on the availability of a guide star and should therefore represent a statistically correct distribution of QSOs characteristics. We should also note that the initial list of QSOs used here is quite biased against a sky coverage concentrated near the Galactic Plane. The HFT would allow observing 6073 targets in $K$, i.e. 38 per cent of the initial list. Among these, 658 targets would have sufficient tracking quality for a good observation in the $J$ band. The HFT would be really needed for an AGN BLR program in the $J$ band with $U T s$. For observations in median seeing conditions, the numbers of accessible targets drop to 832 targets with the GFT and 4267 with the HFT.

Figs 22(a) and 22(b) show the number of targets that could be observed using MATISSE with GFT tracking (left) and an HFT tracker (right). With the GFT, 4881 targets would be accessible to MATISSE, including 3415 well tracked enough for good observations in $L$ and 4878 suitable for good observations in $M$. Here, the number of targets is dominated by the necessity to have a $\lambda / 10$ tracking on the guide star in $K$ to avoid fringe jumps that would be very destructive for $L$ and M observations. The HFT would allow observing 12037 targets, i.e. 76 per cent of the initial list and 10010 targets ( 63 per cent) would be tracked well enough for observations in all MATISSE bands.

With $A T s$, the GFT would allow observing 227 QSOs with MATISSE in $N$ (Fig. 23). This is an extremely small fraction (1.4 per cent) of the initial list but would be quite sufficient for a very significant program. Note that on $A T S$, we only need to install a simple upgrade allowing it to feed MATISSE with a target up to 1 arcminute away from the GFT guide star. The fact that the $A T \mathrm{AO}$ would almost certainly fail to track on these QSOs (we have not checked the range of off-axis tracking for the $\mathrm{AO}$ of the $A T s$ ) is not a showstopper in the $N$ band as $1.8 \mathrm{~m}$ telescopes are diffraction limited at $10 \mu \mathrm{m}$ in good seeing conditions. One might just need a fast tip-tilt tracker at the entrance of MATISSE. With the HFT, a quite large numbers of QSOs would be accessible to MATISSE.

As explained at the end of Section 3.4, the figures for the HFT on the $A T s$ also apply to a new generation interferometer with $1.8 \mathrm{~m}$ apertures on a site similar to Paranal. We see that such an interferometer would have access to a substantial program on AGN torus in the mid-IR with quite enhanced imaging capability with regard to the VLTI but that the near-IR and the visible would be out of reach. If an optimum design of PFI allows an overall transmission of 10 per cent instead of the 2 per cent maximum considered here for the VLTI, this situation could be improved, as indicated by Fig. 24 for an interferometer with 1.8 $\mathrm{m}$ telescopes and $3 \mathrm{~m}$ telescopes. We see that the visible would be accessible only for interferometers with quite large apertures, which quite certainly makes it out of any reasonable expectation. However, an array of $1.8 \mathrm{~m}$ telescopes with optimized transmission would have a potential for the imaging of some BLRs in the $K$ and $H$ bands assuming that there are candidates with sufficient red shifts in the list.

\subsection{A shortlist of nearby AGNs}

We have used the shortlist of 331 nearby (less than $100 \mathrm{Mpc}$ ) AGNs with well-defined characteristics selected by Asmus et al. (2020) to further investigate the feasibility of observations with MATISSE. We will first select the targets that have guide stars suitable for off-axis tracking and observations with MATISSE. As this list was not flux cut in magnitude like the QSO list, some targets may not be bright enough to observe even with the long coherent integration offered by the off-axis tracking.

All sources are brighter than magnitude 17 in the Wise W1 filter $(3.4 \mu \mathrm{m})$, from the wise all-sky survey, except for 18 AGN which had no reported counterpart within 3 arcseconds. From the list of known AGN and R90 AGN candidates, we calculated the predicted angular size in $K$ band. First, we determine the bolometric luminosity for each object using the $12 \mu \mathrm{m}$ luminosity. We convert that luminosity, found in Asmus et al. (2020), into 2-10 KeV luminosity using the MIR-Xray correlation in Asmus et al. (2020) and we then convert the 2-10 KeV luminosity to bolometric using a conversion factor of 10 from Vasudevan et al. (2010). We use the $\Omega_{T}\left(\lambda_{K}\right) \propto L^{1 / 2}$ relation confirmed by Suganuma et al. (2006) where $\Omega_{T}\left(\lambda_{K}\right)$ is the physical radius of the dust as modelled by a thin torus and $L$ is the bolometric luminosity, and $R_{44}$ which is 


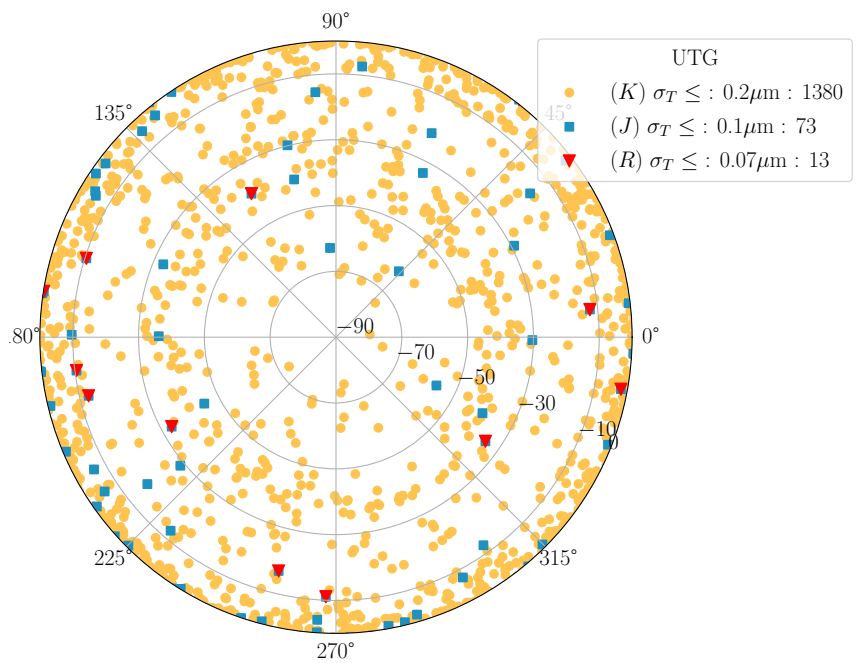

(a)

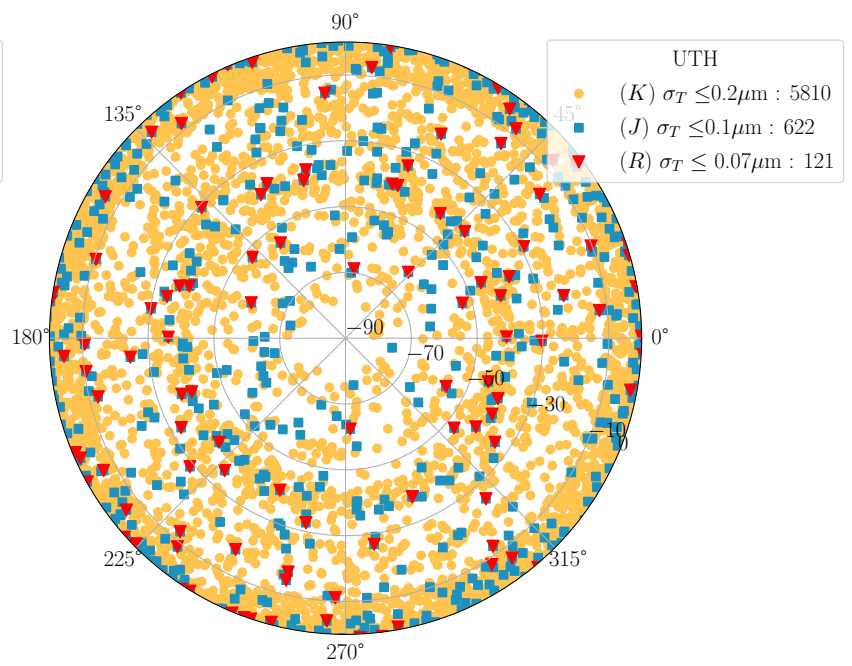

(b)

Figure 21. Targets from our list of 15799 QSOs observable in the near-IR with the UTs with the GFT (a) and the HFT (b). The orange dots are for targets observable only in the $K$ band, the blue squares for targets that would also be observable in the $J$ band, and the red triangles for targets potentially observable in the visible.

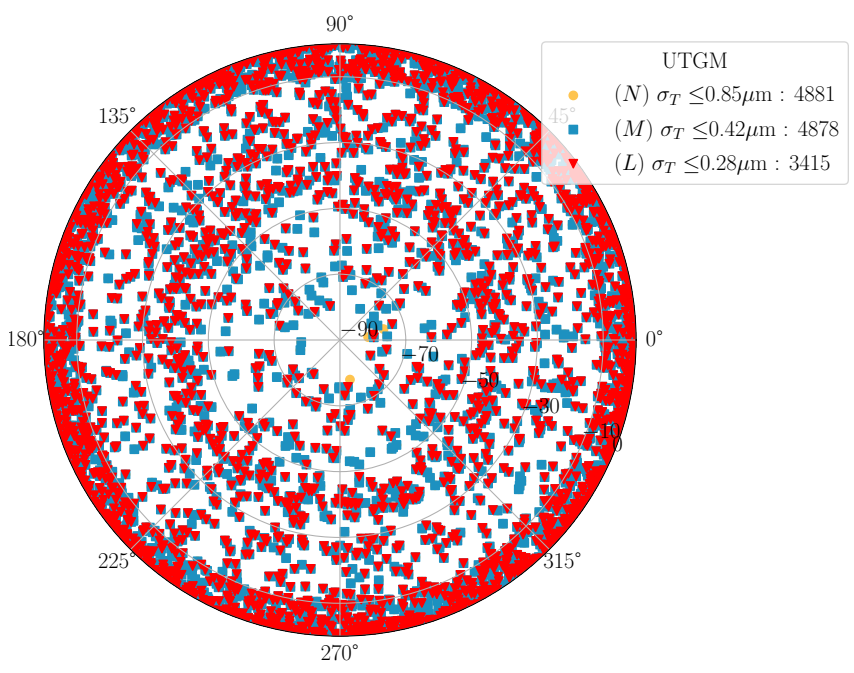

(a)

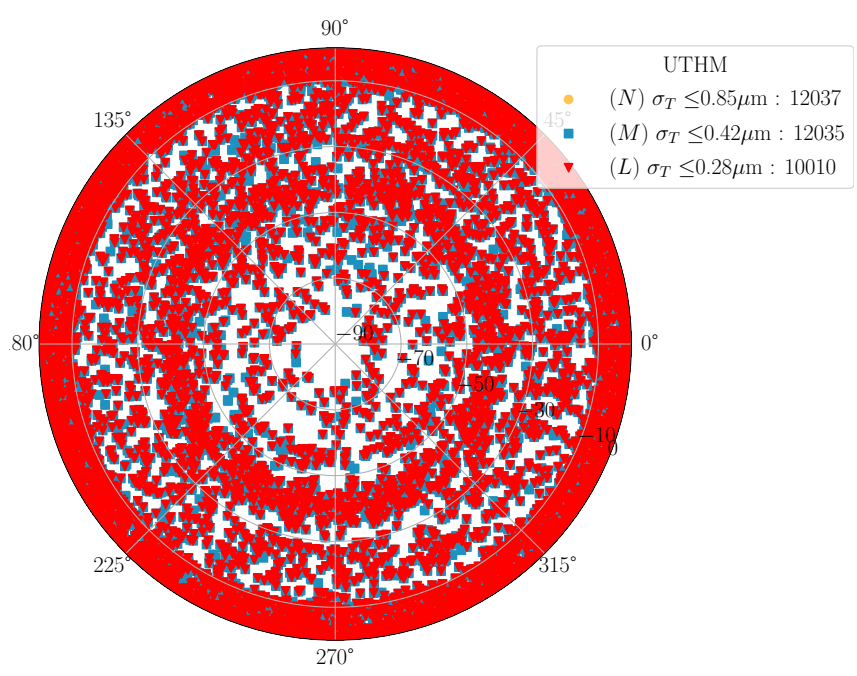

(b)

Figure 22. Targets from our list of 15799 QSOs observable in the mid-IR with MATISSE on the UTs with the GFT (a) and the HFT (b). The red triangles are for targets observable in the $L, M$, and $N$ band, the blue squares for targets observable in the $M$, and $N$ band, and the orange dots for targets observable only in the $N$ band.

the radius at a luminosity of $10 \times 10^{44} \mathrm{erg} / \mathrm{s}$. Using that relation, we calculate the physical radius of the dust in $K$ band and convert the associated diameter to an angular size using the distances provided in Asmus et al. (2020). For angular size in the $N$ band, we use the median size ratio $\frac{\Omega_{T}\left(\lambda_{N}\right)}{\Omega_{T}\left(\lambda_{K}\right)}=9$ established by Burtscher et al. (2013) from $M I D I$ observations. Then we will also use the $\mathrm{N}$ band magnitude of the AGN core found in Asmus et al. (2020) to evaluate the coherent flux SNR that can be achieved on these targets with MATISSE.

The commissioning of MATISSE (Petrov et al. 2020) showed that in the $\mathrm{N}$ band with $U T s$, we obtain a coherent flux $S N R_{C 1}=1$ per coherence time $\left(\mathcal{T}_{C}=250 \mathrm{~ms}\right)$ for each $0.3 \mu \mathrm{m}$ spectral channel, for a $170 \mathrm{mJy}$ source. In $N$, we are always dominated by the background photon noise and the $S N R_{C 1}$ per frame is proportional to the source flux $F(j y)$ in Jansky and to the square root of the integration time: $S N R_{C 1} \propto F(j y) \sqrt{\mathcal{T}_{\text {int }}}$. If $\mathcal{T}_{\text {int }}$ is the integration time allowed by the fringe tracking without fringe jumps and $\mathcal{T}_{\text {tot }}$ is the total open shutter time on the source, the coherent flux $S N R$ will be given by:

$S N R_{C}=\frac{\mathrm{SNR}_{C 1}^{2}}{\sqrt{1+2 \mathrm{SNR}_{C 1}^{2}}} \sqrt{\frac{\mathcal{T}_{\text {tot }}}{\mathcal{T}_{\text {int }}}}$

with

$\mathrm{SNR}_{C 1}=\sqrt{\frac{\mathcal{T}_{\text {int }}}{\mathcal{T}_{c}}} \frac{F(J y)}{0.17}=10^{3.24-0.4 N_{\mathrm{mag}}}$,

with $\mathcal{T}_{\text {int }}=10 \mathrm{~s}, \mathcal{T}_{C}=250 \mathrm{~ms}$, and $\mathcal{T}_{\text {tot }}=360 \cdot \mathcal{T}_{\text {int }}$ we have :

$S N R_{C}=\frac{S N R_{C 1}^{2}}{\sqrt{1+2 S N R_{C 1}^{2}}} \cdot \frac{\tau_{\text {tot }}}{\tau_{\text {int }}}=\frac{10^{7.76-0.8 N_{\mathrm{mag}}}}{\sqrt{1+10^{6.78-0.8 N_{\mathrm{mag}}}}}$,

where we have used $F\left(N_{\text {mag }}=0\right)=47.33$ jy at $9 \mu \mathrm{m}$. 


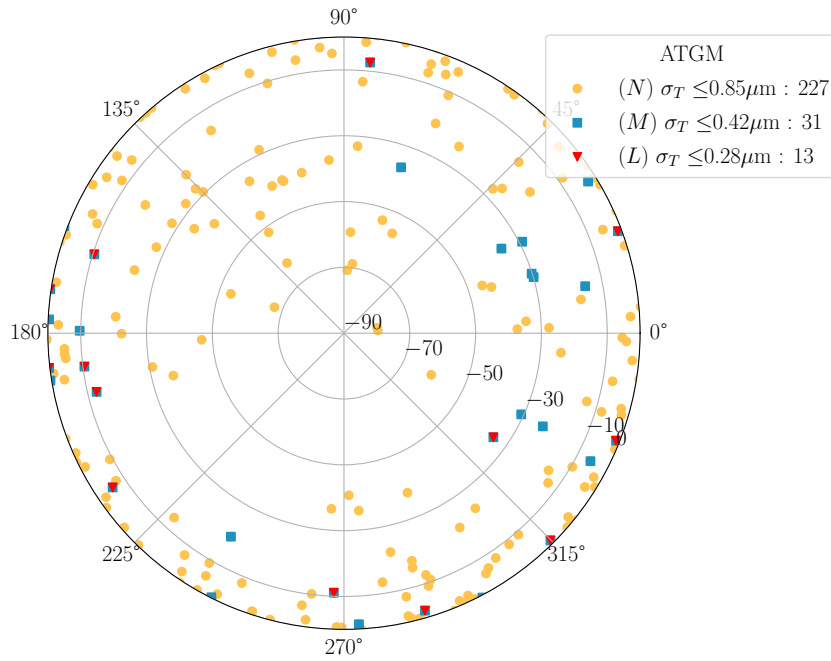

(a)

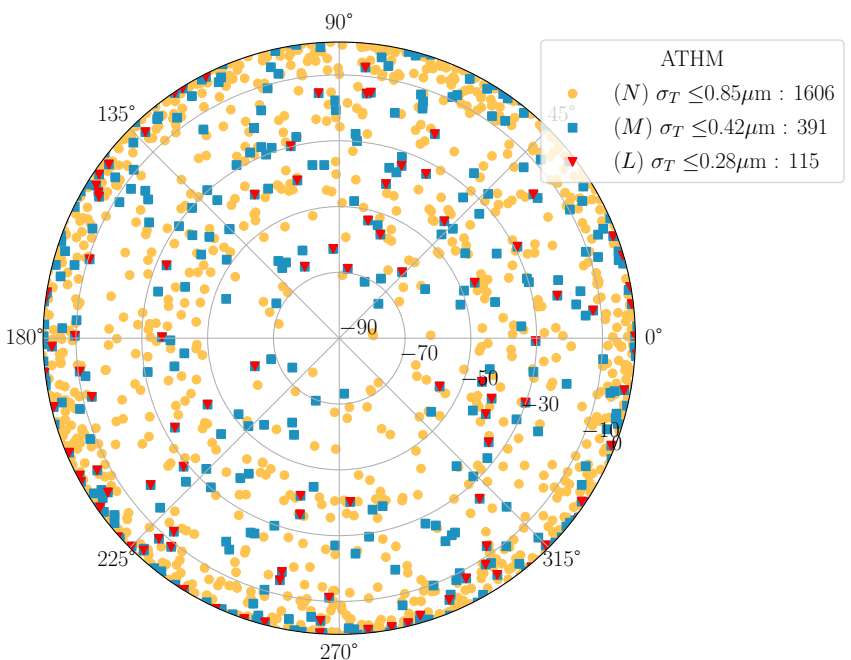

(b)

Figure 23. Targets from our list of 15799 QSOs observable in the mid-IR with the GFT (left) and the HFT (right) on ATs. The orange dots are for targets observable only in the $N$ band, the blue squares for targets observable in the $N$ and $M$ band. The red triangles are for targets observable in the $L, M$, and $N$ band.

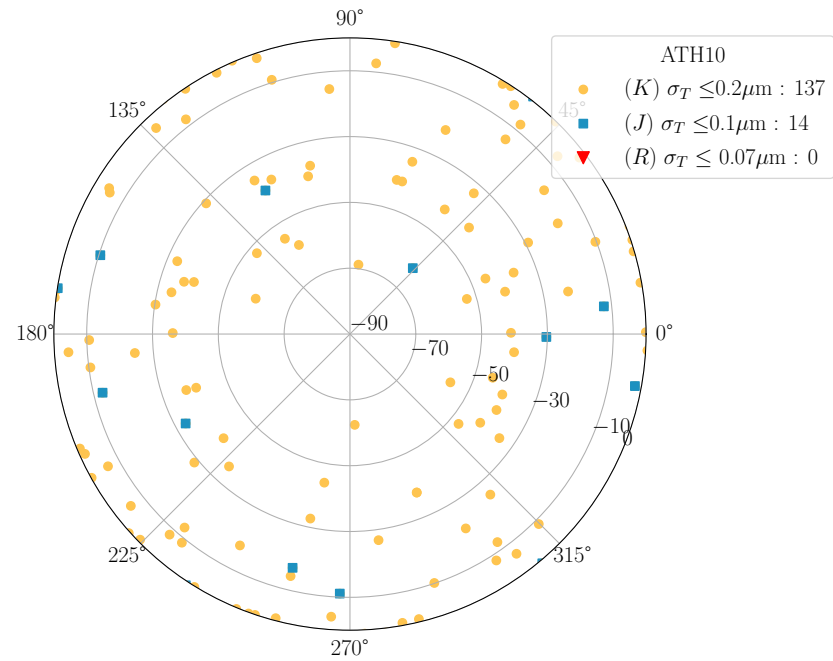

(a)

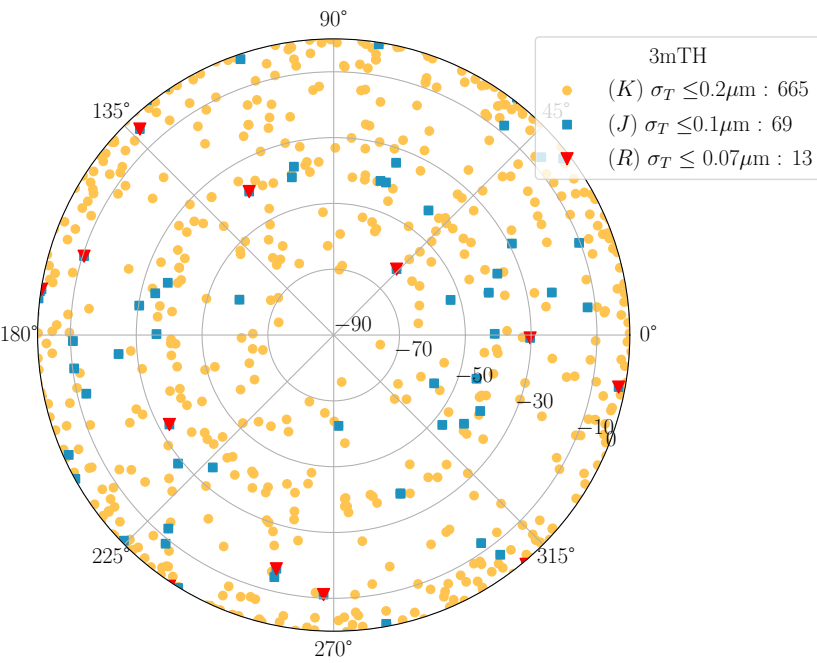

(b)

Figure 24. Targets that would be observable with an HFT on a new generation interferometer with overall transmission 10 per cent and $1.8 \mathrm{~m}$ telescopes (left) and $3 \mathrm{~m}$ telescopes (right) in a site with the same seeing as Paranal. The orange dots are for targets observable only in the $K$ band, the blue squares for targets that would also be observable in the $J$ band, and the red triangles for targets potentially observable also in the visible.

With the $A T s$, we lose three magnitudes even if we integrate slightly longer and the same computation gives:

$S N R_{C}=\frac{S N R_{C 1}^{2}}{\sqrt{1+2 S N R_{C 1}^{2}}} \sqrt{\frac{\tau_{\mathrm{tot}}}{\tau_{\mathrm{int}}}}=\frac{10^{5.36-0.8 N_{\mathrm{mag}}}}{\sqrt{1+10^{4.38-0.8 N_{\mathrm{mag}}}}}$.

Then we use equations $(65,66$, and 67$)$ to find if the object can be used for imaging, for ADI, or only for PDI. If none of these conditions can be satisfied, the source can be fringe tracked but is too faint to obtain usable data in $1 \mathrm{~h}$ with $U T S$ and $2 \mathrm{~h}$ with $A T s$ within our $S N R=10$ criteria.

Fig. 25 shows the nearby AGNs from Asmus et al. (2020) list observable with MATISSE on the UTS. The HFT would allow off-axis tracking with sufficient precision for $N$ band observations on 249 nearby AGNs, i.e. more than 75 per cent of the targets in the list. We would obtain usable information on 162 targets and 152 of them should be large enough for image reconstruction. With the GFT, we would be able to track on 143 targets and to make images in 100 of these. Fig. 26 shows the nearby AGNs observable with ATs. Quite remarkably among the 54 candidates (i.e. 16 per cent) that could be off-axis tracked, 16 will be bright enough for image reconstruction with the very good $u-v$ coverage permitted by the $A T s$. This illustrates again the strong justification of the HFT for MATISSE imaging with the ATs. With the GFT, the number of potential image reconstructions from this list drops to 2 but includes very famous and important targets.

\section{INTERFEROMETRY IN ANTARCTICA}

About $15 \mathrm{yr}$ ago, quite a lot of thinking and site testing has been invested investigating the possibility of a large astronomical facility in Antarctica. The seeing at Dome $\mathrm{C}$ is variable and most often quite poor below the thin turbulent ground layer but it becomes often very good 


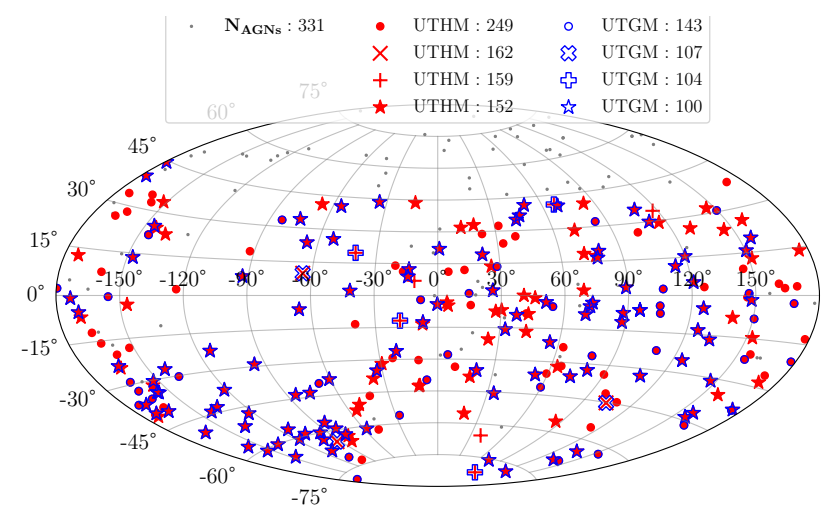

Figure 25. Nearby AGNs that would be observable with MATISSE on the UTS. The red symbols are for targets made accessible by the HFT. The red dots are for AGNs that have an HFT guide star but are too faint to provide useful information in $1 \mathrm{~h}$ of open shutter time. The red $\mathrm{x}$ are for targets restricted to PDI and the red + for targets accessible also by ADI. The red stars are for targets large enough for image reconstruction. The blue symbols have the same meanings for targets that can be observed with the GFT.

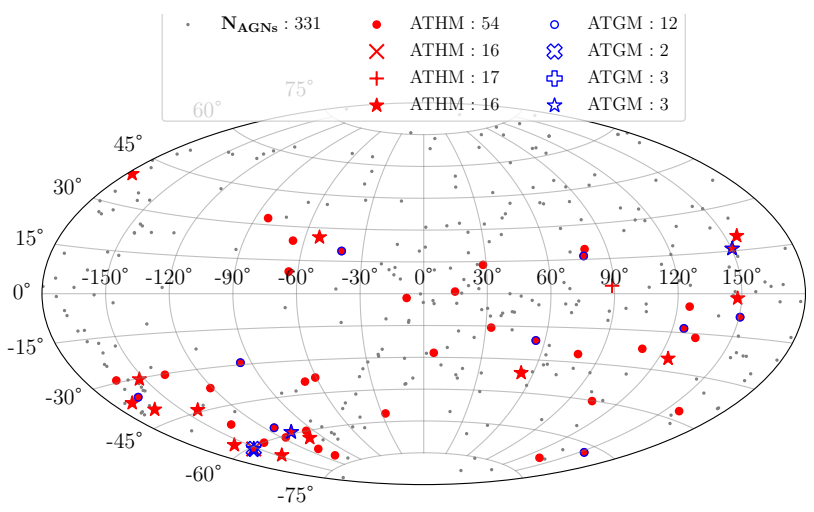

Figure 26. Nearby AGNs that would be observable with MATISSE on the ATs. The symbols are the same as in Fig. 25. On ATs the HFT makes a huge difference.

above $20 \mathrm{~m}$ and almost always excellent above a $30 \mathrm{~m}$. We used the seeing parameters given in Table 3 obtained from Aristidi et al. (2009, 2020). The very small outer scale corresponds to large isopistonic angles even with modest apertures as shown by Fig. 10 where we see that the isopistonic angle at Dome $\mathrm{C}$ with $1.8 \mathrm{~m}$ telescopes would be comparable to this at Paranal with $8 \mathrm{~m}$ telescopes given that the primary mirrors are above the ground layer. At Dome A, the situation could be even better, with a probably finer ground layer Swain \& Gallée (2006). The potentially much larger off-axis potential at Dome $\mathrm{C}$ was one of the initial triggers of this work. The Fig. 27 show the sky coverage that would be obtained with $1.8 \mathrm{~m}$ telescopes for $K$ band and $N$ band observations with an HFT with still 2 per cent overall transmission. The sky coverage is fair in $K$ and looks better than the sky coverage with the $8 \mathrm{~m}$ telescopes in Paranal. For $N$ band observations, we have a very good sky coverage, larger than 20 per cent in all the observable sky. However, Fig. 28 shows the fraction of observable stars in the large QSO list and we see that we still are quite below the possibility of a 8 $\mathrm{m}$ telescope at Paranal. In particular, we note the very small number of targets that would be observable in the visible. Then, even at Dome $\mathrm{C}$, it seems that a network of $1.8 \mathrm{~m}$ telescope would not allow imaging of BLRs in the visible. However, these result might be biased by our QSO list with its the over representation of targets near the equator that would not be observable from Dome C. For YSOs and for observations in the $N$ band in general, we would be limited by the availability of interesting targets a declination below $\sim-30^{\circ}$. It seems indeed that this limitation strongly restricts the number of interesting YSO candidates.

With an interferometer of overall transmission 2 per cent and $1.8 \mathrm{~m}$ apertures, the Fig. 28 shows a high potential of BLR imaging in the $K$ band. Imaging of AGN BLRs in the visible would require larger apertures or a much improved overall transmission even at Dome C.

\section{DISCUSSION AND PERSPECTIVES}

This paper provides tools to estimate the performances of off-axis fringe tracking that include all the atmospheric effects that can impact it. The parametric modelling of the atmospheric effects such as the loop error, related to the evolution of piston with time, and the anisopistonic error, related to the evolution of piston with angular distance, have the great advantage of allowing the estimation of tracking performance for a given off-axis source from standard seeing parameters measured in Paranal, and other observatories, such as the Fried parameters, the coherence time, and the isoplanetic angle.

Our analysis of the loop error due to the piston evolution with time is based on atmospheric perturbations only. We know that real interferometers, in particular the VLTI with $U T S$, are affected by a substantial level of vibration. The GRAVITY+ plan to upgrade the VLTI includes passive and active devices that damp these vibrations. A next step of our work should be to include the vibration power spectrum in the loop error. This would allow a more realistic error budget and consolidate the specifications that the vibration correction should achieve. As the frame time and performances actually achieved by the GFT with the ATs are in quite good agreement with our computation, we tend to believe that the combination of a vibration control that would reduce the $U T$ vibration levels to those of the ATs with Kalman filtering, which allow reducing the impact of vibration on fringe tracking performance, should allow fringe tracking quality on UTs to approach or even slightly exceed the atmospheric limit described here. In spite of average coupling efficiency (Lacour et al.2019) comparable on ATs and $U T s$, the current performance of the GFT on UTs displays a deficit of more than 2.5 magnitudes with regard to these theoretical predictions, which are quite well confirmed by the measured performances on ATs. The SNR analysis given here suggests that most of this loss should be attributed to very fast instabilities of the AO. This is an additional strong argument for the radical upgrade of this subsystem. However, the effective flux imbalance needed to explain the GFT magnitude deficit on UTs looks surprisingly high and should be analysed in more details.

Our analysis of the anisopistonic error is based on atmospheric models and on two campaigns of outer scale measurements at Paranal described in Ziad et al. (2016). Although these atmospheric models are relatively well supported by experimental evidence of AO systems, the specific anisopistonic error has not been measured extensively. A coordinated campaign, combining anisopistonic measurements thanks to the prototype GRAVITY + wide-field combiner tested at the VLTI with a seeing sensor including outer scale measurements would be very valuable to check the predictions of this paper and give the parameters to actually evaluate the performance of off-axis tracking. As the current GSMs are expected to allow a $2 \mathrm{~h}$ forecast of all the observing parameters (Giordano et al. 2021), the formalism developed here might lead to a forecast of off-axis performance and hence a major optimization of VLTI large programs. In Section 4.5, we have seen that moving from the median seeing to the best 20 per cent seeing can change the number of targets that can be fringe tracked off-axis by 40 per cent. A proper evaluation of the off-axis fringe tracking performances from the standard seeing parameters would therefore be highly valuable. 


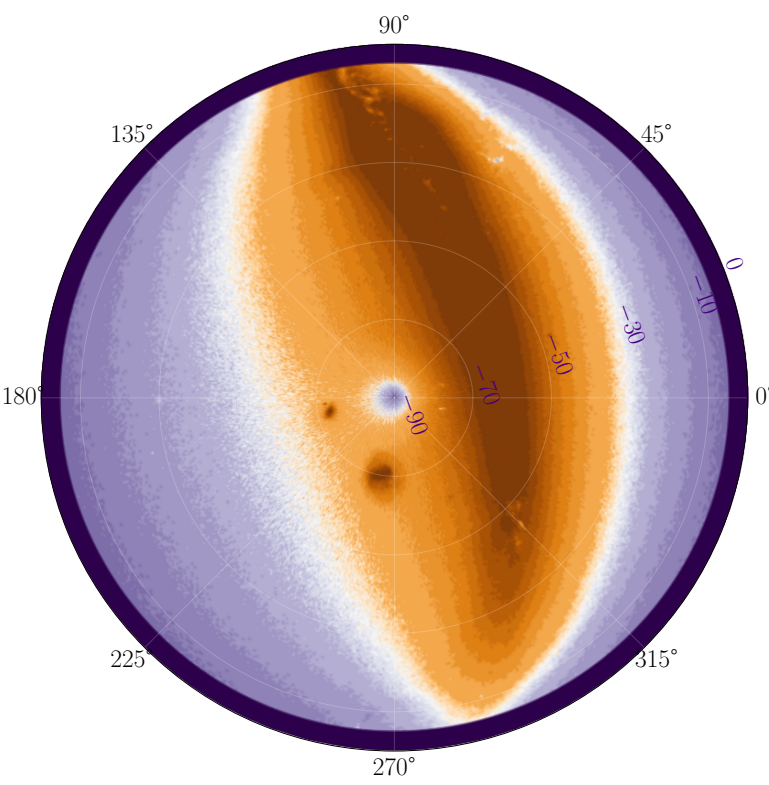

(a) FT-AT Dome C

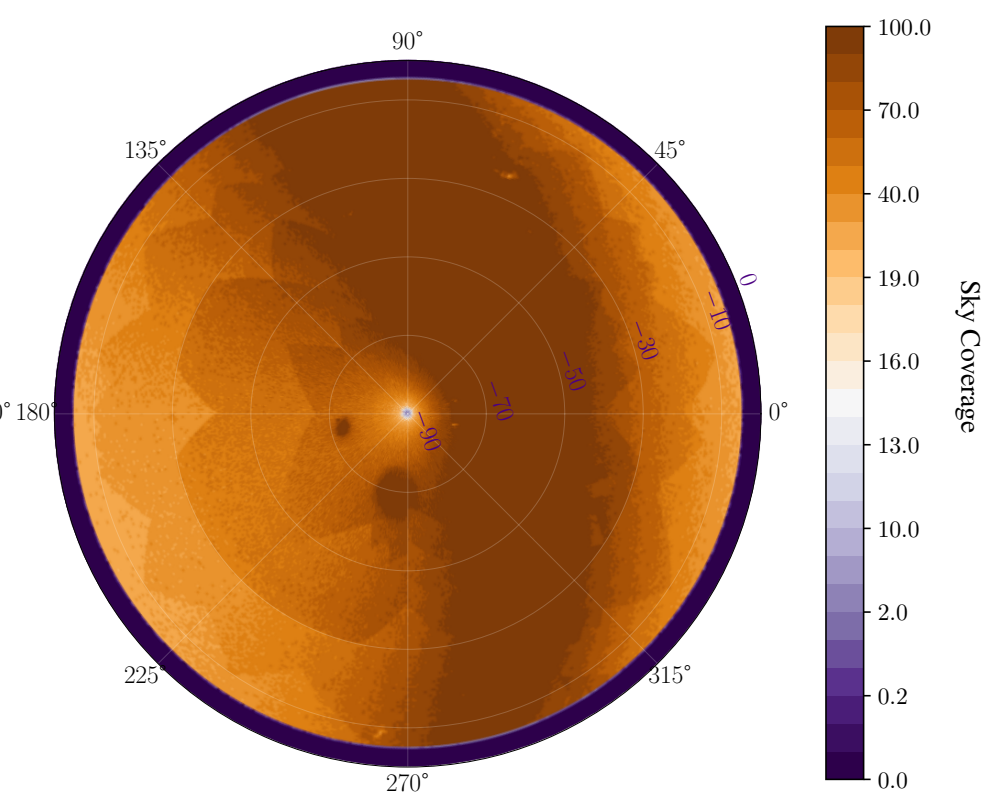

(b) HFT-AT MATISSE Dome C.

Figure 27. Sky coverage with an HFT on a Dome C interferometer with $1.8 \mathrm{~m}$ telescopes for observations in the near-IR (right) and in the mid-IR (left)
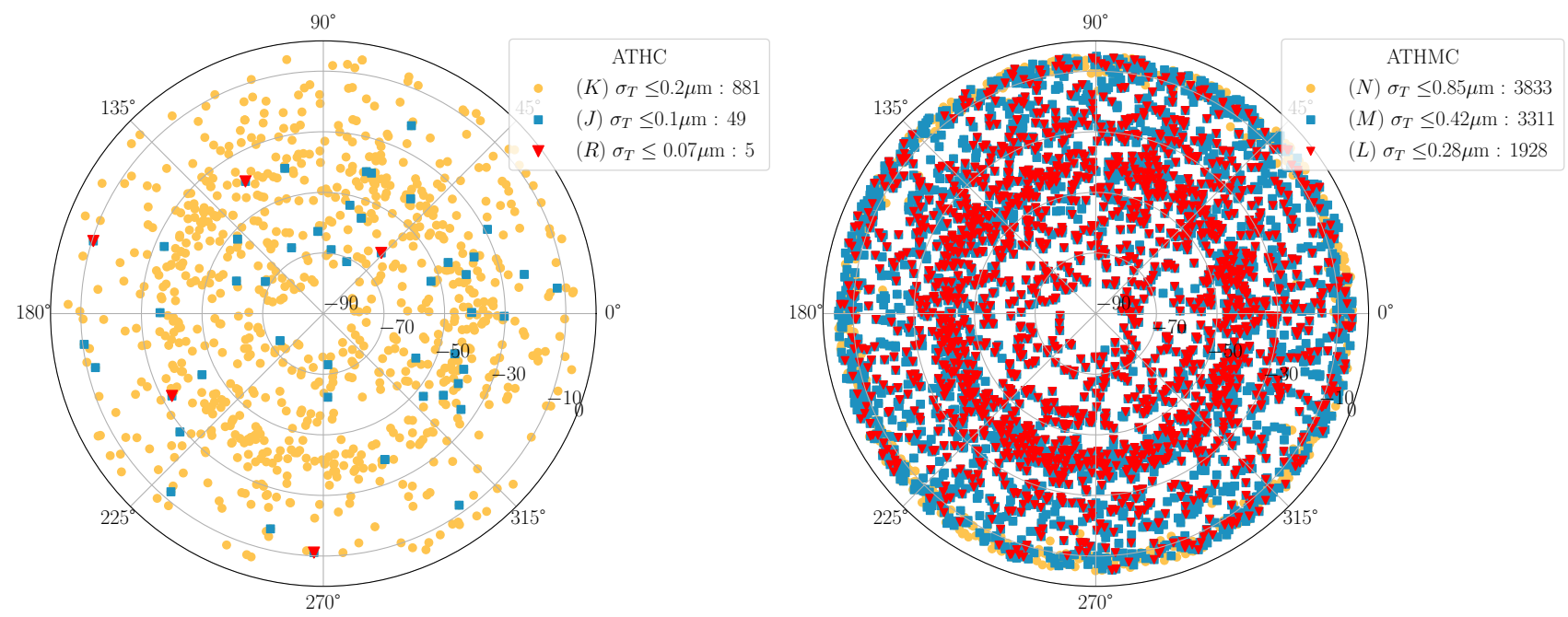

Figure 28. QSOs observable with an HFT on a Dome C interferometer with $1.8 \mathrm{~m}$ telescopes. Left: observations in the near-IR in K (orange dots), H\&K (blue squares) and J\&H\&K (red triangles). Right: observations in the mid-IR in N (orange dots), M\&N (blue squares), and L\&M\&N (red triangles).

This paper underlines the very large potential of off-axis fringe tracking in the near-IR for observations in the mid-IR. The anisopistonic angle in the $N$ band with the $U T S$ can approach three arcminutes, and the design of the wide-field system that feeds sources with large angular separations in the VLTI instrument(s) and tracker chain should take that parameter in consideration. We have set the tracking requirement in $K$ band at $0.2 \mu \mathrm{m}$. This requirement could be substantially relaxed in terms of FT variance. However, for tracking in near-IR and for observations in the mid-IR, it is crucial to analyze the probability of one $\lambda$ fringe jumps in the tracker that would impact the contrast and ruin the calibration in the science instrument. With a $\lambda / 10$ specifications, the fringe jumps should be extremely rare. Investigating the tracking accuracy that would allow an acceptable fringe jump rate will be a key element to optimize the off-axis tracking for the mid-IR.

Finally, we have compared the potential sky coverage with our best estimate for the current GFT as it could be improved by the GRAVITY+ upgrades and an improved FT combining a larger spectral band, a slightly improved transmission, and an optimized concept. The sky coverage with the GFT appears quite sufficient for the large AGN program that is one of the roots of the GRAVITY+ proposal because there is a large enough number of AGNs all over the sky to provide more than enough candidates. For others, more specific and rarer targets, the very substantial gain in sky coverage provided by the HFT would be very valuable. The HFT would be decisive for efficient off-axis tracking with the ATs.

\section{CONCLUSION}

We have developed a complete analysis of the error budget of off-axis fringe tracking in the near-IR for observations in the near-IR and the mid-IR. We have applied it to the VLTI with the ATs almost as they are and with the $U T S$ as they should be after the GRAVITY + upgrade project. We have considered two FTs: the GFT with its current transmission, 
detector, and $K$ band operation as well as a new generation FT with a larger bandpass covering partially the $J, H$ and $K$ bands with a slightly increased transmission and a new concept of HFT. The contribution of the variation of the atmospheric piston with time and angular separation to the off-axis tracking error has been based on analytic parameters based on the standard seeing sensor measures. The chromatic dependence of the piston, when the tracking and observations are performed at different wavelengths has been evaluated from a Keck Interferometer model updated by recent and ongoing MATISSE and GRAVITY measurements. We have combined our estimates of the off-axis tracking error budget and parameters with a database of more than $8 \times 10^{8}$ stars, extracted from the Gaia second data release, to compute sky coverage maps for the VLTI with UTS and ATs for the GFT and the HFT in different seeing conditions. With the GFT, the sky coverage with UTs is better than 10 per cent in a domain extending from $\pm 20^{\circ}$ to $30^{\circ}$ away from the Galactic Plane, depending on seeing conditions. With the HFT the sky coverage with UTS is larger than 10 per cent in most of the sky observable from Paranal. To give a more specific example of a large AGN program on the VLTI, we have selected a sample of more than 15000 QSOs from the VHS and we have evaluated the number of QSOs accessible to observations in all bands from $J$ to $N$. The first conclusion is that the GFT in the GRAVITY+ context will give access to more than enough targets for its large AGN program in the $K$ band. The second conclusion is that off-axis fringe tracking has an enormous potential for observations in the mid-IR with MATISSE that should be considered in GRAVITY + and MATISSE with GRAVITY + science programs. To be more specific about the AGN program of MATISSE, we have analysed the potential performances of MATISSE on a list of 331 nearby AGNs for which we have detailed information. For 10 per cent of these targets with the GFT and 30 per cent of these targets with the HFT, the SNR and the resolution of MATISSE should be sufficient to obtain detailed images of the dust torus in N. The third conclusion is that the HFT would offer a very substantial increase in sky-coverage and tracking precision that would be decisive for many programs including AGN observations with the ATs. We have also evaluated the potential of a new generation interferometer, like the proposed PFI, for off-axis tracking. We show that with an HFT, whose tracking performance does not decrease with the number of apertures, an array of $1.8 \mathrm{~m}$ telescopes at a site of Paranal quality, the PFI would be able to track off-axis for fine imaging in the $N$ band of many YSOs.

\section{ACKNOWLEDGEMENTS}

(i) This work has been supported by the French government through the Université Côte d'Azur Jedi (UCAJEDI) Investments in the Future project managed by the National Research Agency (ANR) with the reference number ANR-15-IDEX-01.

(ii) This work has been supported by the Chinese Academy of Sciences, through a grant to the Chinese Academy of Sciences South America Center for Astronomy (CASSACA) in Santiago, Chile.

(iii) This research has made use of the SIMBAD database, operated at Centre de Données de Strasbourg (CDS), Strasbourg, France ( 'The SIMBAD astronomical database' Wenger et al. 2000).

\section{DATA AVAILABILITY}

This paper is using publicly available data from the SIMBAD astronimical database and the GAIA second Data Release.

\section{REFERENCES}

Antonucci R., 1993, ARA\&A, 31, 473

Aristidi E., et al., 2009, A\&A, 499, 955
Aristidi E., Agabi A., Abe L., Fossat E., Ziad A., Mékarnia D., 2020, MNRAS, 496,4822

Asmus D., et al., 2020, MNRAS, 494, 1784

Astropy Collaboration 2018, AJ, 156, 123

Blind N., Absil O., Le Bouquin J. B., Berger J. P., Chelli A., 2011, A\&A, 530, A121

Burtscher L., et al., 2013, A\&A, 558, A149

Colavita M. M., et al., 2013, PASP, 125, 1226

Eisenhauer F., 2019. GRAVITY+: Towards faint science, Presented in The Very Large Telescope in 2030 (VLT2030). ESO, Garching, Germany, p. 30

Elhalkouj T., Petrov R. G., Lazrek M., Ziad A., Benkhaldoun Z., 2006, in Monnier J. D., Schöller M., Danchi W. C., eds, Proc. SPIE Conf. Ser. Vol. 6268, Advances in Stellar Interferometry. SPIE, Bellingham. p. 626812

Elhalkouj T., Ziad A., Petrov R. G., Lazrek M., Elazhari Y., Benkhaldoun Z., 2008, A\&A, 477, 337

Evans D. W., et al., 2018, A\&A, 616, A4

Femenia B., Carbillet M., Esposito S., Riccardi A., 2000, in Léna P., Quirrenbach A., eds, Proc. SPIE Conf. Ser. Vol. 4006, Interferometry in Optical Astronomy. SPIE, Bellingham. p. 1116

Finger G., et al., 2016, in Marchetti E., Close L. M., Véran J.-P., eds, Proc. SPIE Conf. Ser. Vol. 9909, Adaptive Optics Systems V, SPIE Bellingham. p. 990912

Fried D. L., 1982, J. Opt. Soc. of Am (1917-1983), 72, 52

Gai M., et al., 2004, in Traub W. A., ed., Proc. SPIE Conf. Ser. Vol. 5491, New Frontiers in Stellar Interferometry. SPIE, Bellingham. p. 528

Gaia Collaboration 2018, A\&A, 616, A1

Ginsburg A., et al., 2019, AJ, 157, 98

Giordano C., Rafalimanana A., Ziad A., Aristidi E., Chabé J., Fanteï-Caujole Y., Renaud C., 2021, MNRAS, 504, 1927

Gravity Collaboration 2017a, The Messenger, 170, 10

Gravity Collaboration 2017b, A\&A, 602, A94

Gravity Collaboration 2018, Nature, 563, 657

Gravity Collaboration 2020a, A\&A, 633, A110

Gravity Collaboration 2020b, A\&A, 634, A1

Gravity Collaboration 2020c, A\&A, 635, A92

Harris C. R., et al., 2020, Nature, 585, 357

Hönig S. F., Watson D., Kishimoto M., Hjorth J., 2014, Nature, 515, 528

Houairi K., Cassaing F., Perrin G., Eisenhauer F., Brandner W., Straubmeier C., Gillessen S., 2008, in Schöller M., Danchi W. C., Delplancke F., eds, Proc. SPIE Conf. Ser. Vol. 7013, Optical and Infrared Interferometry. SPIE, Bellingham. p. 70131B

Ireland M., 2019. Unravelling Planet Formation, Presented in The Very Large Telescope in 2030 (VLT2030). ESO, Garching, Germany, p. 34

Kirchschlager F., Ertel S., Wolf S., Matter A., Krivov A. V., 2020, MNRAS, 499, L47

Kishimoto M., Hönig S. F., Antonucci R., Barvainis R., Kotani T., Tristram K. R. W., Weigelt G., Levin K., 2011, A\&A, 527, A121

Koresko C., Colavita M. M., Serabyn E., Booth A., Garcia J., 2006, in Monnier J. D., Schöller M., Danchi W. C., eds, Proc. SPIE Conf. Ser. Vol. 6268, Advances in Stellar Interferometry SPIE, Bellingham. p. 626817

Lacour S., et al., 2019, A\&A, 624, A99

Leinert C., et al., 2004, A\&A, 423, 537

Lindegren L., et al., 2018, A\&A, 616, A2

Lopez B., et al., 2014, The Messenger, 157, 5

Mathar R. J., 2007, J. Opt. A: Pure Appl. Opt., 9, 470

Matter A., Pignatale F. C., Lopez B., 2020, MNRAS, 497, 2540

McMahon R. G., Banerji M., Gonzalez E., Koposov S. E., Bejar V. J., Lodieu N., Rebolo R., VHS Collaboration 2013, The Messenger, 154, 35

Meisner J. A., Jaffe W. J., Le Poole R. S., 2012, in Delplancke F., Rajagopal J. K., Malbet F., eds, Proc. SPIE Conf. Ser. Vol. 8445, Optical and Infrared Interferometry III. SPIE, Bellingham. p. 84451L

Monnier J. D., et al., 2018, Exp. Astron., 46, 517

Papoulis A., 1965, Probability, random variables and stochastic processes. McGraw-Hill, New York

Parenti R. R., Sasiela R. J., 1994, J. Opt. Soc. Am. A, 11, 288

Petrov R. G., 1989, in Alloin D. M., Mariotti J. M., eds, Diffraction-Limited 
Imaging with Very Large Telescopes Vol. 274, NATO Advanced Study Institute (ASI) Series C. p. 249

Petrov R., Roddier F., Aime C., 1986, J. Opt. Soc. Am. A, 3, 634

Petrov R. G., et al., 2001, C.R. Acad. Sci., Paris, 2, 67

Petrov R. G., et al., 2007, A\&A, 464, 1

Petrov R. G., et al., 2014, in Rajagopal J. K., Creech-Eakman M. J., Malbet F., eds, Proc. SPIE Conf. Ser. Vol. 9146, Optical and Infrared Interferometry IV. SPIE, Bellingham. p. 91462P (arXiv: 1410.4822)

Petrov R. G., Boskri A., Bresson Y., Agabi K., Folcher J.-P., Elhalkouj T., Lagarde S., Benkhaldoum Z., 2016, in Malbet F., Creech-Eakman M. J., Tuthill P. G., eds, Proc. SPIE Conf. Ser. Vol. 9907, Optical and Infrared Interferometry and Imaging V. SPIE, Bellingham. p. 99071F (arXiv: 1610.08287)

Petrov R., et al., 2019. A new generation fringe tracker, Presented in The Very Large Telescope in 2030 (VLT2030). ESO, Garching, Germany, p. 37

Petrov R. G., et al., 2020, in SPIE ed., Conf. Ser. Vol. 11446, Optical and Infrared Interferometry and Imaging VII. SPIE. p. 114460L

Rakshit S., Petrov R. G., Meilland A., Hönig S. F., 2015, MNRAS, 447, 2420

Sasiela R. J., 1994, J. Opt. Soc. Am. A, 11, 379

Skrutskie M. F., et al., 2006, AJ, 131, 1163

Suganuma M., et al., 2006, ApJ, 639, 46

Swain M. R., Gallée H., 2006, in Stepp L. M., ed., Proc. SPIE Conf. Ser. Vol. 6267, Ground-based and Airborne Telescopes. SPIE, Bellingham. p. $62671 \mathrm{~K}$

Takato N., Yamaguchi I., 1995, J. Opt. Soc. Am. A, 12, 958

Vannier M., et al., 2004, in Traub W. A., ed., Proc. SPIE Conf. Ser. Vol. 5491, New Frontiers in Stellar Interferometry. SPIE, Bellingham. p. 577

Vannier M., Petrov R. G., Lopez B., Millour F., 2006, MNRAS, 367, 825

Varga J., et al., 2021, A\&A, 647, A56

Vasudevan R. V., Fabian A. C., Gandhi P., Winter L. M., Mushotzky R. F., 2010, MNRAS, 402, 1081

Wang J.-M., Songsheng Y.-Y., Li Y.-R., Du P., Zhang Z.-X., 2020a, Nat. Astron., 4, 517

Wang T.-W., et al., 2020b, MNRAS, 499, 4068

Wenger M., et al., 2000, A\&AS, 143, 9

Ziad A., Elhalkouj T., Petrov R. G., Borgnino J., Lazrek M., Benkhaldoun Z., Martin F., Elazhari Y., 2016, MNRAS, 458, 4044

This paper has been typeset from a $\mathrm{T}_{\mathrm{E}} \mathrm{X} / \mathrm{L} \mathrm{T} \mathrm{E} \mathrm{X}$ file prepared by the author. 